\title{
Low Field MRI and the Development of Polarized Nuclear Imaging (PNI) - A New Imaging Modality
}

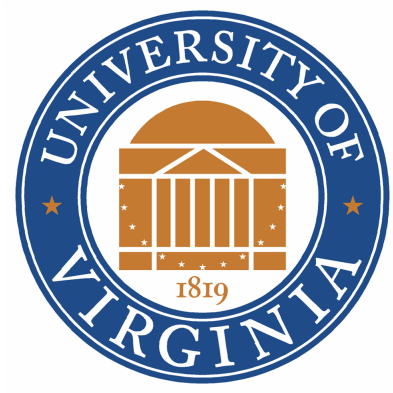

\author{
Yuan Zheng \\ Department of Physics \\ University of Virginia
}

A thesis submitted for the degree of

Doctor of Philosophy

September 2014 
Advisor

Member 1

Member 2

Outside Member
Gordon D. Cates, Jr

Thomas F. Gallagher

Mark B. Williams

G. Wilson Miller $\overline{5}$


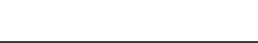

Day of the defense: 
C)Copyright by Yuan Zheng

All Rights Reserved.

September 2014 


\begin{abstract}
In this thesis, we used the technique of Spin-Exchange Optical Pumping (SEOP) to produce hyperpolarized noble gases. We studied the pressure and temperature dependence of ${ }^{129}$ Xe polarization by making a series of cells with different gas compositions and measuring the Xe polarization over a large temperature range.

We constructed a low field MRI scanner for hyperpolarized noble gases. Our scanner was built with off-the-shelf electronics and co-axial circular coils. We imaged both ${ }^{3} \mathrm{He}$ and ${ }^{129} \mathrm{Xe}$ samples. Using a fully phase encoded pulse sequence and with some auxiliary measurements, we acquired a high quality image with ${ }^{129} \mathrm{Xe}$ at low field $(\sim 2 \mathrm{mT})$.

We developed genuinely new techniques of Polarized Nuclear Imaging (PNI) and Polarized Nuclear Detection (PND), which make use of the asymmetric radioactive emissions of certain polarized radioactive nuclei. In these techniques, the nuclear spins are manipulated using conventional Nuclear Magnetic Resonance (NMR) methods. However, instead of detecting radiofrequency electromagnetic waves, single photons (or other types of radioactive emissions) are detected. As a result, the signal detection sensitivity is greatly enhanced. We also developed the novel Cates-Miller-Zheng (CMZ) pulse sequence class that is specially suited for PNI, and successfully acquired a preliminary image of a ${ }^{131 \mathrm{~m}}$ Xe sample.
\end{abstract}


To Yishen, for your love, support, and understanding 


\section{Acknowledgements}

First of all, I would like to give my deepest thanks to my advisor, Gordon Cates, not only for his support and guidance throughout my graduate studies, but also for setting an excellent example for me as a person. I'm proud to have worked with him for five years.

I am also deeply grateful to my co-advisor Wilson Miller, from whom I learned almost everything I know about MRI. His sense of humor adds a lot of fun to our research, and I will never forget his help when my car broke down (because he then fried my battery!).

I'd like to thank Prof. Tom Gallagher and Prof. Mark Williams for spending time reading my thesis and serving on the defense committee from their very busy time schedule.

I'd like to thank Dr. Al Tobias and Dr. Vladimir Nelyubin for their help on my research project. Al played an essential role in filing and handling the cells studied in this thesis. And more importantly, I learned from him the right attitude for conducting experiments: be as careful as possible and don't rush. Vladimir is definitely an expert in lasers and mechanical designing. His help is very important in setting up some of the experiments. I'd like to thank recent graduates of Cates Lab: Scott Rohrbaugh, Jaideep Singh, Peter Dolph and Karen Mooney. Their theses have always been a great source of reference for me, and I'm still benefiting from some software and hardware that they set up. Thanks is also due to all current graduate students in Cates group.

I owe a lot of thanks to Prof. Rick Marshall, Prof. Donal Day, Prof. Don

Crabb, Prof. Nilanga Liyanage, Prof. Dinko Pocanic and Nikolay Sandev from the Physics Demo Lab for generously lending me lots of lab equipment. 


\section{Contents}

List of Figures Xiii

List of Tables

1 Introduction 1

1.1 An Overview of Conventional MRI . . . . . . . . . . . . . 1

1.2 MRI with Hyperpolarized Gases . . . . . . . . . . . . . . 2

1.3 Motivations of Polarized Nuclear Imaging (PNI) and Polarized Nuclear

Detection $(\mathrm{PND}) \ldots \ldots \ldots \ldots \ldots$

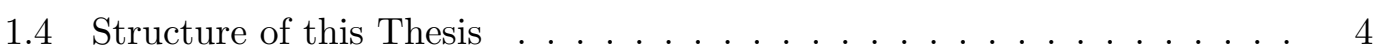

2 Spin-Exchange Optical Pumping 5

2.1 An Overview of SEOP . . . . . . . . . . . . . . 6

2.2 Optical Pumping . . . . . . . . . . . . . . . 7

$2.2 .1 \quad \mathrm{Rb}$ in $\mathrm{SEOP} \ldots \ldots \ldots \ldots$

2.2.2 Basic Processes of Optical Pumping . . . . . . . . 8

2.2.3 Optics Setup for Optical Pumping . . . . . . . . . . . . 10

2.2.4 Solving for Polarization . . . . . . . . . . . . . 11

2.2 .5 Optical Pumping Rate . . . . . . . . . . . . . 12

2.2.6 Rb Spin Destruction Rate . . . . . . . . . . . . . . . 14

2.2.7 More about Optical Pumping . . . . . . . . . . . . 16

2.2.7.1 Nuclear Slowing Down Factor . . . . . . . . . . 16

2.2.7.2 Laser Polarization and Skew Angle. . . . . . . . 17

2.2.7.3 Wall Relaxation ............. 18

2.3 Spin Exchange and Relaxation of Noble Gas Nuclei . . . . . . . . . . . . 18

2.3.1 Spin Exchange Rate . . . . . . . . . . . . . . . . 19 
2.3.2 Noble Gas Spin Relaxation Rate . . . . . . . . . . . . 21

2.4 SEOP of ${ }^{131 \mathrm{~m}} \mathrm{Xe} \ldots \ldots \ldots \ldots \ldots . \ldots \ldots$

3 Xe Polarization Studies and Xe Polarimetry 27

3.1 NMR Techniques . . . . . . . . . . . . . . . . . . . . 28

3.1 .1 Spins in a Magnetic Field . . . . . . . . . . . . 28

3.1.2 The Rotating Reference Frame . . . . . . . . . . . . . 28

3.1.3 Adiabatic Fast Passage . . . . . . . . . . . . . . 30 30

3.1 .4 Pulsed NMR . . . . . . . . . . . . . . . . . 32

3.2 Electron Paramagnetic Resonance Polarimetry . . . . . . . . . . . . 34

3.3 Xe-129 Polarization at Different Pressures and Temperatures . . . . . . 37

3.3.1 Cell Preparation . . . . . . . . . . . . . . . 37

3.3 .2 SEOP of the Cells . . . . . . . . . . . . . 39

$3.3 .3{ }^{129}$ Xe Polarimetry . . . . . . . . . . . . . . 41

3.3.3.1 EPR Technical Details ............ 41

3.3.3.2 Pulsed NMR Technical Details . . . . . . . . . . 46

3.3.3.3 Calibration Constants . . . . . . . . . . . 49

3.3.3.4 Errors of the Calibration Constants . . . . . . . . 52

3.3.4 Results and Discussions ............... . . 54

4 Low Field MRI of HP Gases

4.1 Basics of MRI . . . . . . . . . . . . . . 5 58

4.2 Introduction of Low Field HP Gas MRI . . . . . . . . . . . . . 59

4.3 Low Field HP Gas MRI Setup . . . . . . . . . . . . . . . . . . 61 61

4.3.1 The Holding Field Coil, RF Coil, and Detection Coils . . . . . 61

4.3.2 The Gradient Coils . . . . . . . . . . . . . . . . . . 63 63

4.3.3 Control and Data Acquisition Electronics . . . . . . . . . 68

4.3.4 Laser-Polarized Phantoms . . . . . . . . . . . . . . . . . 68

4.3.5 Field Shimming and Transmitter Calibration . . . . . . . . . 70

4.4 Imaging Methods . . . . . . . . . . . . . . . . . . . . 71

4.4 .1 Pulse Sequences . . . . . . . . . . . . . . . 71

4.4 .2 Auxiliary Measurements . . . . . . . . . . . . . 74

4.4 .3 Image Reconstruction . . . . . . . . . . . . . . . 77

4.5 Results and Discussion . . . . . . . . . . . . . 78 
$4.5 .1 \quad$ MR Images . . . . . . . . . . . . . . . . . . 78

4.5.2 Advantages of a Fully Phase-Encoded Image Acquisition . . . . . 80

4.5.3 In Vivo MRI at Low Fields . . . . . . . . . . . . . . . . 82

\section{Polarized Nuclear Imaging (PNI) and Polarized Nuclear Detection} (PND) 85

5.1 Radiation Asymmetry from Aligned Nucleus . . . . . . . . . . . 86

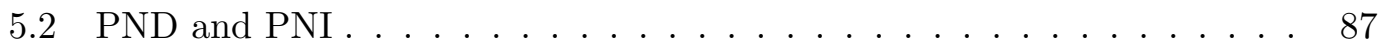

5.2.1 Polarized Nuclear Detection (PND) _ . . . . . . . . . . 87

5.2 .2 Basics of Polarized Nuclear Imaging (PNI) _ . . . . . . . . 89

5.2 .3 PNI with a CMZ Pulse Sequence . . . . . . . . . . . . . . 91

5.3 PNI Setup . . . . . . . . . . . . . . . . . . 96

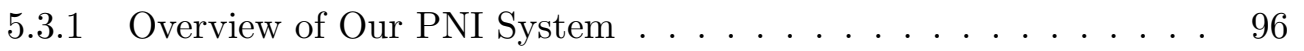

5.3 .2 Detectors and Electronics . . . . . . . . . . . . . . . 97

5.3 .3 Phantom Preparation . . . . . . . . . . . . . . . . . 99

5.4 Imaging Methods . . . . . . . . . . . . . . . . . 102

5.4 .1 Basic PND measurements . . . . . . . . . . . . . . . 102

5.4.1.1 NMR Spectroscopy . . . . . . . . . . . . . . . . 102

5.4.1.2 Spin Evolution During SEOP . . . . . . . . . . . . 103

5.4.1.3 RF Calibration . . . . . . . . . . . . . . . . . 104

5.4.1.4 Free Induction Decay . . . . . . . . . . . . . . 105

5.4.1.5 CMZ Delay Study . . . . . . . . . . . . . . . . 106

5.4 .2 Pulse Sequence . . . . . . . . . . . . . . . . . . . 107

5.4 .3 Auxiliary Measurements . . . . . . . . . . . . . . . . . . 108

5.4 .4 Image Reconstruction . . . . . . . . . . . . . . . . . . 109

5.5 Results and Discussions . . . . . . . . . . . . . . . 111

5.6 Other Applications of PND . . . . . . . . . . . . . . . . 112

6 Conclusion 117

6.1 Summary . . . . . . . . . . . . . . . . 117

6.2 Future Directions . . . . . . . . . . . . . . . . . . 117

References 


\section{List of Figures}

2.1 Basic setup of the SEOP system in our lab. . . . . . . . . . . . . 6 6

2.2 Energy levels of the spin $3 / 2{ }^{87} \mathrm{Rb}$ (not to scale) $\ldots \ldots \ldots$

2.3 Simplified schematic of optical pumping. . . . . . . . . . . . . . 10

2.4 Optics for generating circularly polarized laser light for SEOP . . . . . . 11

2.5 Two Mechanisms for spin exchange between Rb and noble gas nuclei . . 20

3.1 Precession of a spin around a holding magnetic field . . . . . . . . . . 29

3.2 Evolution of the effective field in the rotating frame during an AFP measurement. . . . . . . . . . . . . . . . . 31

3.3 A typical AFP measurement in our lab. . . . . . . . . . . . 32

3.4 Schematic of a Pulsed NMR measurement. . . . . . . . . . . . . . . 33

3.5 A glass string with 4 cells. . . . . . . . . . . . . . . . . 38

3.6 Measuring the laser absorption spectrum at the back of the heated oven. 40

3.7 Laser spectrum measured behind cell X4 . . . . . . . . . . . . . 41

3.8 Our Setup for EPR polarimetry and Pulsed NMR. . . . . . . . . . . 42

3.9 A Lorentzian function and its derivative. . . . . . . . . . . . . . . 44

3.10 An real FM sweep taken in our lab. . . . . . . . . . . . . . . . 44

3.11 The Proportional-Integral Feedback circuit. . . . . . . . . . . . . . . 45

3.12 An EPR frequency shift measurement. . . . . . . . . . . . . . . . 46

3.13 A spinup curve taken with cell $\mathrm{X} 2$ at $70^{\circ} \mathrm{C}$ with 3 spectrally narrowed lasers. . . . . . . . . . . . . . . . . . 4 47

3.14 Free Induction Decay signal taken with cell $\mathrm{X} 4$ at $68^{\circ} \mathrm{C}$ with 3 spectrally narrowed lasers. . . . . . . . . . . . . . . . . . . . . . . . . . . . . . 48

3.15 Tipangle measurements taken with cell $\mathrm{X} 4$ at $68^{\circ} \mathrm{C}$ with 3 spectrallynarrowed lasers. . . . . . . . . . . . . . . . . . . . 49 49 
3.16 Xe polarization as a function of temperature for cells X1-4. . . . . 54

4.1 An overview of our low-field MRI apparatus . . . . . . . . . . . . 62

4.2 Observing the Gradient coils from different angles. . . . . . . . . . . . . 65

4.3 $B_{z}$ and its gradient generated by the actual y gradient coils per Ampere per turn in the $\mathrm{x}-\mathrm{y}$ plane. . . . . . . . . . . . . . . . . 67

4.4 Low field imaging system schematic . . . . . . . . . . . . . . . 69

4.5 Phantoms that we imaged in our low field scanner. . . . . . . . . . . . 70

4.6 A FID with shimmed holding field . . . . . . . . . . . . . 71

4.7 A Typical gradient echo pulse sequence. . . . . . . . . . . . . . . 72

4.8 Fully phase encoded pulse sequence . . . . . . . . . . . . . . 74

4.9 Holding field drift in ${ }^{129} \mathrm{Xe}$ precession frequency during a fully phase encoded imaging procedure. . . . . . . . . . . . . . . 75

4.10 Control voltage and the current response of our $y$ gradient coil for the maximum phase-encoding gradient . . . . . . . . . . . . 776

4.11 Measured $\mathrm{k}_{y}$ values with $\mathrm{k}_{x}=0$, its linear fit and residuals $\ldots . . . . \quad 77$

4.12 Images acquired by gradient echo acquisitions . . . . . . . . . . . . . 79

4.13 Fully phase encoded images with different levels of post-processing . . . 80

5.1 The Fourier coefficients of the ${ }^{131 \mathrm{~m}} \mathrm{Xe} 164 \mathrm{keV}$ gamma emissions as a function of polarization. . . . . . . . . . . . 87

5.2 Angular distribution of ${ }^{131 \mathrm{~m}} \mathrm{Xe} 164 \mathrm{keV}$ gamma rays with $0 \%$ and $100 \%$ polarization. . . . . . . . . . . . . . . . . . . . 88

5.3 Count rate asymmetry from precessing ${ }^{131 \mathrm{~m}}$ Xe nuclei. . . . . . . . . . 89

5.4 The CMZ pulse sequence. . . . . . . . . . . . . . . 92

5.5 Spin motion during a CMZ sequence . . . . . . . . . . . . 93

5.6 ${ }^{131 \mathrm{~m}} \mathrm{Xe} 164 \mathrm{keV}$ gamma emission anisotropy as a function of the detector polar angle. . . . . . . . . . . . . . . . . 95

5.7 Detectors set along the longitudinal and transverse directions. . . . . . 97

5.8 A NaI detector with a lightguide. . . . . . . . . . . . . . 98

5.9 Polarized and unpolarized ${ }^{131 \mathrm{~m}}$ Xe decay spectra measured by the longitudinal detector. . . . . . . . . . . . . . . . . . . . . 999

5.10 PNI signal processing schematic. . . . . . . . . . . . . . 100 
5.11 Removing the "dirty" gases of the ${ }^{131 \mathrm{~m}}$ Xe gas mixture by sending it into a temporary cell with Rb. . . . . . . . . . . . . . . 101

5.12 Transferring radioactive Xe to a phantom. . . . . . . . . . . . 102

5.13 Longitudinal gamma ray count rate as a function of the transverse RF frequency. . . . . . . . . . . . . . . . . . . 103

5.14 Change of the longitudinal gamma ray count rate during SEOP. . . . . 104

5.15 Ratio of the count rate immediately after and before the RF pulse as a function of RF amplitude. . . . . . . . . . . . . . . . 105

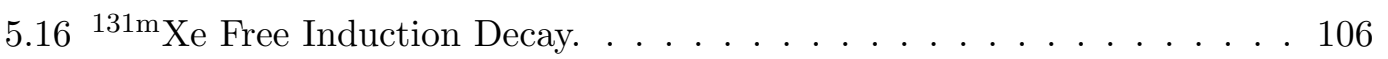

5.17 Ratio of the gamma count rates as a function of additional delay. . . . . 107

5.18 Holding field drift measured by EPR during the whole CMZ procedure. 109

5.19 Corrected k space data of the longitudinal detector. . . . . . . . . . . . 110

5.20 Images acquired by the longitudinal and transverse detectors . . . . . . 110

5.21 PNI image of a $\sim 3 \mathrm{~cm}$ diameter sphere. . . . . . . . . . . . . . 111

5.22 Rabi precession spectrum of ${ }^{131 \mathrm{~m}}$ Xe measured by a longitudinal detector. 113

5.23 Spin precession and decay during a ${ }^{131 \mathrm{~m}} \mathrm{Xe} \mathrm{T}_{1 \rho}$ measurement. . . . . . . 114

$5.24 \mathrm{~T}_{1 \rho}$ dispersion curve of $131 \mathrm{~m}$ Xe. . . . . . . . . . . . . . 115 


\section{List of Tables}

2.1 Pressure broadening and shift coefficients for common SEOP gases . . . 13

3.1 Selected nuclear species with their spin, gyromagnetic ratio and magnetic moment ........................... 28

3.2 2" Xe Cells X1-X4 volumes and contents . . . . . . . . . . . . . . 40 40

3.3 Polarization calibration constants and their errors for Xe cells. . . . . 53 


\section{1}

\section{Introduction}

\subsection{An Overview of Conventional MRI}

The development of Magnetic Resonance Imaging (MRI) started about half a century ago. The first MRI image was published in 1973 by Paul Lauterbur [1], which only showed two capillaries filled with water. Several years later, images of a living human were acquired: Peter Mansfield imaged a human finger in 1976 and published the image in 1977 [2. Also in 1977, Raymond Damadian performed a human chest scan[3]. The first commercial system was introduced in 1980. By 2011 there were around 12740 MRI scanners in the United States (OECD Health Data 2011) and this number keeps increasing.

Magnetic Resonance Imaging (MRI) is a powerful technique that is widely used in diagnosis of disease processes nowadays. It has lots of advantages:

- MRI is a non-invasive technique, and it also doesn't include any radiation.

- MRI can generate image contrast based on the spin relaxation properties of body substances, and is particularly useful for the scanning and detection of abnormalities in soft tissue structures.

- MRI scanners can produce images in any plane without physically moving the subject or any part of the scanner.

- MRI can provide information about how the blood moves through certain organs and blood vessels, thus enabling the detection of blood circulation problems. 
However, every technique has its disadvantages:

- MRI scanners are very expensive. The cost of each scanner is about 3 million dollars. This means the number of scanners are usually limited and the cost of an MRI scan is high. The cost of an MRI scan typically ranges from $\$ 1200$ to $\$ 4000$, which is usually more expensive than other examining methods, like CT scans and X-rays.

- MRI scans are not comfortable. A scan typically takes 30 mins, and the patient is placed in an enclosed space with loud noises caused by the fast changing gradients. Therefore, it often causes claustrophobia in susceptible patients.

- Patients with pacemakers or metal implants are contraindicated due to possible injury or image distortion.

- Conventional MRI doesn't work well on lung imaging due to the low proton density and the magnetic susceptibility variation at the air/tissue interface. Besides, proton MRI cannot be used to evaluate lung function.

\subsection{MRI with Hyperpolarized Gases}

A new branch of MRI-Hyperpolarized Gas MRI was developed in 1994 [4], and has proven to be particularly useful for functional imaging of the lung. Unlike conventional MRI which uses the proton thermal polarization, this technique employs pre-polarized noble gases. The noble gases (usually ${ }^{3} \mathrm{He}$ or ${ }^{129} \mathrm{Xe}$ ) are first polarized by the technique of Spin-Exchange Optical Pumping (SEOP) [5, 6, 7]. Then when inhaled, the magnetization of noble gases can be used to acquire an MRI image. Since the polarization of polarized noble gases is on the order of $10^{5}$ times larger than the water thermal polarization in a normal commercial scanner, the MR signal size is large despite the low physical density of the gas, permitting good visualization of the lung airspaces.

It is worth pointing out that the polarization of the noble gases is independent of the $\mathrm{B}_{0}$ holding field strength, which makes it possible to perform MRI at a much lower holding field ( $\mathrm{mT}$ instead of $\mathrm{T}$ ). Therefore in principle it is possible to replace the massive super-conducting magnets usually seen in commercial scanners by simple solenoids, 
which not only cuts down the cost of a scanner but also brings some advantages unique to low field operations, like more uniform holding field and better penetration of metals.

The most widely used noble gases for hyperpolarized gas MRI are ${ }^{3} \mathrm{He}$ and ${ }^{129} \mathrm{Xe}$. The latter of which is particularly interesting since Xenon is lipophilic, and is well known to dissolve into the blood stream. Therefore, hyperpolarized ${ }^{129} \mathrm{Xe}$ can in principle be used to image parts of the body other than lungs.

\subsection{Motivations of Polarized Nuclear Imaging (PNI) and Polarized Nuclear Detection (PND)}

There are two problems with hyperpolarized Xe imaging: First, it's very difficult to polarize large quantities of xenon to a high polarization. Although some commercial xenon polarizers are available and can polarize reasonable amount of xenon to tens of percents polarization [8, 9, 10, 11], they are very expensive machines and are not available to every lab. Second, although in principle Xe dissolves into blood and can be used to generate contrasts in organs other than the lung, it is sometimes difficult to introduce a big enough amount of xenon to induce a measurable signal, either by inhalation or injection.

We have developed a new technique that we call Polarized Nuclear Imaging (PNI). This technique utilizes trace quantities of hyperpolarized radioactive isotopes of noble gases such as ${ }^{131 \mathrm{~m} X e .}$ Radiation emissions are measured instead of radio-frequency electro-magnetic waves. The amount of radioactive nuclei involved is small enough that they can be readily polarized to a high polarization. In addition to that, because of the small quantities involved here, the radioactive isotopes can be easily introduced into any tissues that are well perfused and used to probe a variety of physiological processes.

PNI is based on the more general technique of Polarized Nuclear Detection (PND). When certain radioactive nuclei are aligned, radiation emissions are spatially asymmetric [12]. MR properties $\left(\mathrm{T}_{1}, \mathrm{~T}_{2}\right.$, resonance frequency, ...) of the radioactive nuclei can be measured using the anisotropic radiation emission. Conventional NMR techniques measure the the weak radio-frequency waves from a collection of rotating spins. On the other hand, gamma rays from the disintegration of radioactive nuclei can be detected individually. Therefore PND is a much more sensitive technique. PND has the 
potential to be used as an non-imaging diagnostic technique that measures the change of MR properties $\left(\mathrm{T}_{1}, \mathrm{~T}_{2}\right.$, resonance frequency, etc.) of the contrast agent in different chemical environment.

\subsection{Structure of this Thesis}

This thesis covers the production and characterization of hyperpolarized noble gases, low field imaging of ${ }^{3} \mathrm{He}$ and ${ }^{129} \mathrm{Xe}$, and the novel PNI/PND techniques. Chapter 2 talks about the basic theories of SEOP. Chapter 3 describes several widely used NMR and polarimetry techniques, and presents the results of our Xe polarization studies. Chapter 4 introduces low-field MRI of hyperpolarized noble gases. Both the setup and pulse sequences are described. Methods for correcting for some system imperfections of our home-built low field scanner are also included. Chapter 5 discusses the novel PNI modality and introduces the platform technique behind it - Polarized Nuclear Detection (PND). Preliminary images acquired by PNI are also presented. Finally, this thesis is summarized and future directions are suggested in Chapter 6. 
2

\section{Spin-Exchange Optical Pumping}

Nuclear spin-polarized noble gases have been found to be useful in many fields. In electron-scattering experiments, polarized ${ }^{3} \mathrm{He}$ is often used as a target material [13, 14]. In recent development in MRI, polarized ${ }^{3} \mathrm{He}$ and ${ }^{129} \mathrm{Xe}$ have been used as the imaging media.

In all of these applications, a nuclear polarization approaching the order of unity is achieved via either Metastability-Exchange Optical Pumping (MEOP) or Spin-Exchange Optical Pumping (SEOP). In comparison, the thermal polarization in a $\sim 1 \mathrm{~T}$ field is five orders of magnitude smaller. MEOP can only be used to polarize ${ }^{3} \mathrm{He}$. It is an efficient process, but ${ }^{3} \mathrm{He}$ is polarized at a relatively low pressure $(\sim 1$ Torr $)$, and further compression of the gas is needed [15]. On the other hand, the technique of SEOP can be used to polarize both helium and xenon, and the total gas pressure can be on the order of several atmospheres ( 1000 Torr).

In our lab, we seal noble gases in glass cells and polarize them by SEOP. For ${ }^{3} \mathrm{He}$ cells of a few atms, we've measured polarizations as high as $70 \%$ [16]. The achievable xenon polarization strongly depends on the xenon pressure in the cell. For cells with several atms of xenon, we can only polarize it to several percent. However, as the xenon pressure is dropped to $\sim 50$ Torr, we have reached a polarization close to $80 \%$. In this chapter, we'll talk about the basics of SEOP. 


\subsection{An Overview of SEOP}

The basic SEOP setup in our lab is shown in Fig. 2.1. The noble gas, usually ${ }^{3} \mathrm{He}$ or ${ }^{129} \mathrm{Xe}$, is sealed in a glass cell together with some alkali metal, a small amount of $\mathrm{N}_{2}$ and maybe some other buffer gases. The cell is placed in the center of a heated oven during SEOP, and a relatively weak ( $\sim 13$ Gauss) holding field is used to define the axis of polarization. Circularly polarized laser beams illuminate the cell, and the noble gas nuclei can be polarized along or against the holding field direction.



Figure 2.1: Basic setup of the SEOP system in our lab. The cell is placed inside the oven and not shown here.

SEOP is a two step process. The first step is optical pumping. The alkali metal atoms, whose density is determined by the oven temperature, are polarized by the circularly polarized photons. The second step is spin exchange. The alkali metal polarization is transferred to the nuclei of the noble gas during spin-exchange collisions that can either be binary, or in the case of Xe, result in the formation and breakup of van der Waals (vdW) molecules. We will describe these two steps separately in the next two sections. 


\subsection{Optical Pumping}

\subsubsection{Rb in SEOP}

Optical pumping is a technique in which circularly polarized light is used to polarize both the electron spin $S$ and the nuclear spin $I_{a}$ of various atoms [17]. Perhaps the most widely used atom in optical pumping is Rubidium. Although in our lab sometimes an alkali hybrid of $\mathrm{Rb} / \mathrm{K}$ is involved for ${ }^{3} \mathrm{He}$ target cells, $\mathrm{Rb}$ is the alkali metal that is directly polarized by the laser light, and its polarization is then transferred to Potassium[16]. Rb is suitable for optical pumping for several reasons. The main advantage is that $\mathrm{Rb}$ has a low melting point $\left(\sim 39.5^{\circ} \mathrm{C}\right)$, which makes it relatively easy to achieve a dense $\mathrm{Rb}$ vapor at an elevated temperature. The $\mathrm{Rb}$ vapor density can be estimated by an updated version of Killian's vapor pressure formula [18]:

$$
[\mathrm{Rb}]=\frac{10^{3.55-4132 / T}}{k_{B} T}
$$

where $[\mathrm{Rb}]$ is the $\mathrm{Rb}$ density in $\mathrm{cm}^{-3}$ and $k_{B}$ is Boltzmann's constant, $\mathrm{T}$ is the temperature in Kelvin. Usually our oven temperature is between $85^{\circ} \mathrm{C}$ to $235^{\circ} \mathrm{C}$. $[\mathrm{Rb}]$ increases quickly with temperature. At $85^{\circ} \mathrm{C},[\mathrm{Rb}] \sim 2.1 \times 10^{12} \mathrm{~cm}^{-3}$. At $180^{\circ} \mathrm{C}$, $[\mathrm{Rb}] \sim 4.3 \times 10^{14} \mathrm{~cm}^{-3}$. At $235^{\circ} \mathrm{C},[\mathrm{Rb}] \sim 3.7 \times 10^{15} \mathrm{~cm}^{-3}$. Another practical reason is the availability of laser power. There are off-the-shelf high power laser packages available at the Rb D1 line wavelength, and they can be readily circularly polarized and used to polarize Rb atoms. Also, the Rb D1 (794.7 nm) and D2 (780 nm) lines are well separated, so even lasers that are spectrally fairly broad can be used to optically pump the $\mathrm{Rb} \mathrm{D} 1$ line without inducing transitions corresponding to the D2 line.

Using $\mathbf{S}$ as the electron spin and $\mathbf{I}$ as the nuclear spin of alkali metal atoms, the Hamiltonian for ground state (orbital momentum $\mathrm{L}=0$ ) alkali metal atoms in a holding field $B_{0}$ along $\mathrm{z}$ direction is [5]:

$$
H=A \mathbf{I} \cdot \mathbf{S}+g_{s} \mu_{B} S_{z} B_{0}-\frac{\mu_{I}}{I} I_{z} B_{0}
$$

where $A \mathbf{I} \cdot \mathbf{S}$ describes the coupling of the nuclear spin $\mathbf{I}$ to the electron spin $\mathbf{S}$. The isotropic magnetic-dipole coupling coefficient is A. The second term gives the coupling of the electron spin to the external field $B_{0}$, where $g_{s} \approx 2$ is the $g$ value of the electron, and $\mu_{B}=9.274 \times 10^{-24} \mathrm{~J} / \mathrm{T}$ is the Bohr magneton. The third terms is for the interaction 
between the nuclear spin and the external field, where $\mu_{I}$ is the nuclear magnetic moment.

The typical $B_{0}$ field used for SEOP in our lab is $\sim 13$ Gauss. As a result, the hyperfine interaction (first term in Eq. 2.2 dominates over the Zeeman interactions (second and third term in Eq. 2.2), and the eigenstates of $\mathrm{H}$ are also the eigenstates of the total angular momentum $\mathbf{F}=\mathbf{S}+\mathbf{I}$ and its projection $F_{z}$ along the external field.

$\mathrm{Rb}$ has two stable isotopes ${ }^{85} \mathrm{Rb}$ and ${ }^{87} \mathrm{Rb}$ with natural abundance $72.2 \%$ and $27.8 \%$. ${ }^{85} \mathrm{Rb}$ has a nuclear spin of $5 / 2$ and ${ }^{87} \mathrm{Rb}$ has a nuclear spin of $3 / 2$. Both of them are used in SEOP. The energy levels of ${ }^{87} \mathrm{Rb}$ are shown in Fig. 2.2 .

\subsubsection{Basic Processes of Optical Pumping}

If we ignore the alkali metal nuclear spin for a moment, the process of SEOP can be described as in Fig. 2.3. Circularly polarized laser light tuned to the D1 line of Rb illuminates the cell along the holding-field direction, and electrons are excited from the $5 S_{1 / 2}$ state to the $5 P_{1 / 2}$ state. While either circular polarization of the laser light works for SEOP, we assume here that the photons carry " +1 " angular momentum, shown as $\sigma^{+}$in Fig. 2.3. Due to the selection rules, only electrons in the $-1 / 2$ spin state will be excited. By colliding with other buffer gases, the two $5 P_{1 / 2}$ states will be mixed and have the same population. The electron then decays back to the two $5 S_{1 / 2}$ states with equal probability. The net effect of such a cycle is that one electron is excited from the $S_{z}=-1 / 2$ state, but it only has $50 \%$ percent probability of coming back to the initial state. For the other $50 \%$ probability it will up in the $S_{z}=+1 / 2$ state. Continuous pumping on the $S_{z}=-1 / 2$ state will eventually deplete the population in that state, and most electrons will be in the $S_{z}=+1 / 2$ state, and we say the Rb is spin polarized. If photons have opposite circular polarization, the electrons will be pumped from the $S_{z}=+1 / 2$ state to the $S_{z}=-1 / 2$ state and $\mathrm{Rb}$ will be polarized to the opposite state.

One thing worth noting is that after being excited and mixed in the $5 P_{1 / 2}$ states, the electrons can decay back to the ground state either by emitting a photon or quenching collisions. Decaying by emitting a photon is harmful for SEOP, since photons from spontaneous decay are unpolarized and can depolarize other $\mathrm{Rb}$ atoms. It is very common to add a small amount (on the order of 100 Torr) of $\mathrm{N}_{2}$ in the cell, which absorbs the energy of $5 P_{1 / 2}$ electrons into its vibrational modes and greatly suppresses 


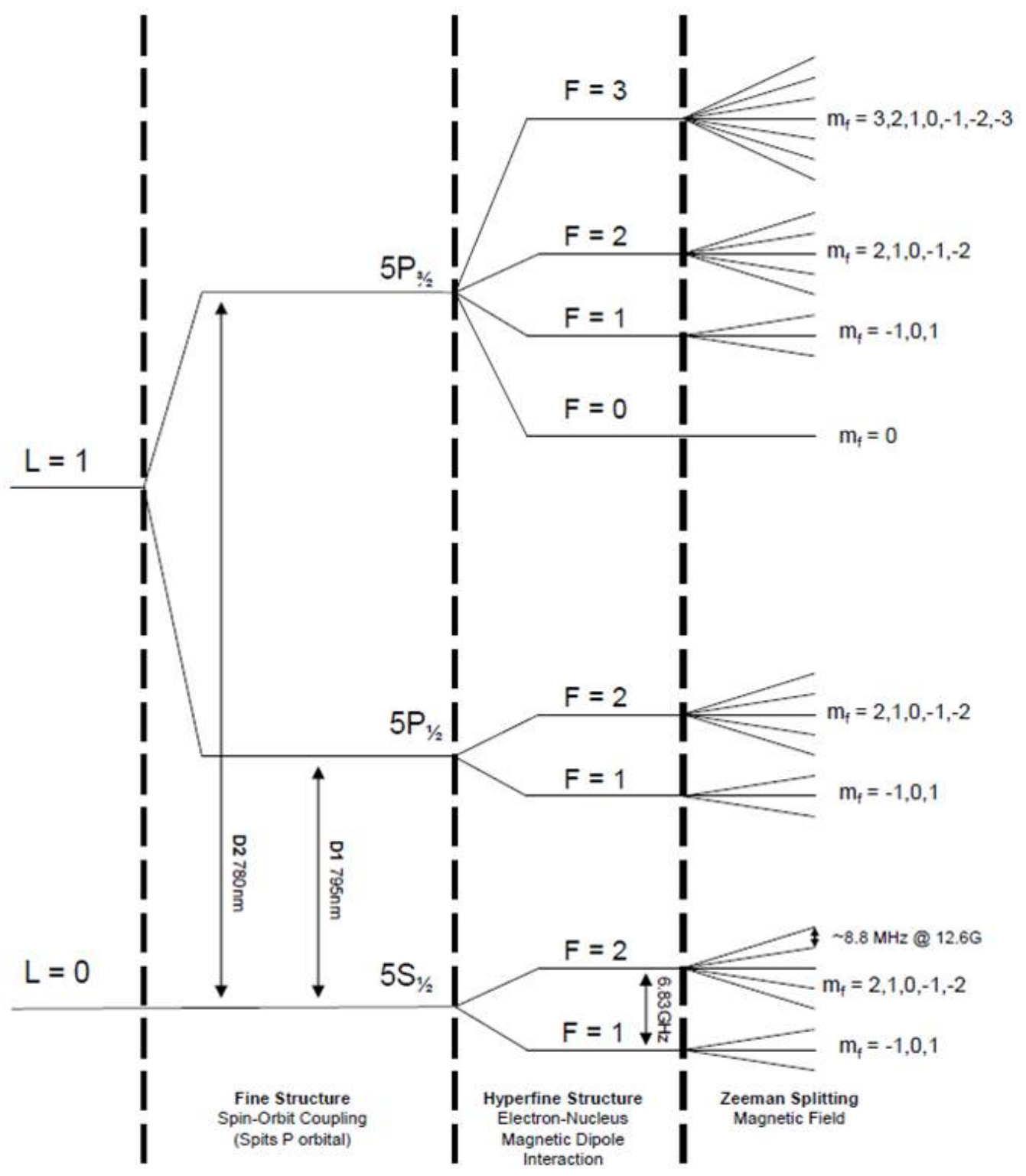

Figure 2.2: Energy levels of the spin $3 / 2{ }^{87} \mathrm{Rb}$ (not to scale) (Adapted from Dolph's PhD thesis [19]) 




Figure 2.3: Simplified schematic of optical pumping. Here the Rb spin is neglected.

the radiative decay. The branching ratio of the radiative decay is given by [20]

$$
P_{\text {rad }}=\frac{3}{3+p_{N_{2}}}
$$

where $p_{N_{2}}$ is the nitrogen pressure at $300 \mathrm{~K}$ in Torr. As an example, 100 Torr $N_{2}$ suppresses the photon emitting branch to about $3 \%$.

\subsubsection{Optics Setup for Optical Pumping}

In our lab, we typically use spectrally narrowed laser systems for SEOP. We have two types of laser systems. One is the Comet laser (Newport Corp., Irvine, CA), and the other is the D-light laser (Raytum Corp., Manassas, VA). Both laser spectra are centered at around the Rb D1 line. The FWHM of the Comet laser is about $0.2 \mathrm{~nm}$, and that of the D-Light laser is about $0.3 \mathrm{~nm}$. The power from each laser is about 20 W. The laser light is circularly polarized using the optics shown in Fig. 2.4.

L1 and L2 are convex lenses used to control the size of the laser beam, so that the laser power is not too focused in the polarizing cube to avoid causing damages, and the beam size is suitable for illuminating the cell. The polarizing cube splits the incident light into two beams with orthogonal polarizations. The beam that goes through has a polarization parallel to this plane, and the beam reflected to the right is polarized perpendicular to the plane. The reflected beam is sent through a quarter wave plate, reflected by a mirror, then returned through the same quarter wave plate. At this point its linear polarization has been rotated to parallel to the plane. Thus it can go through 


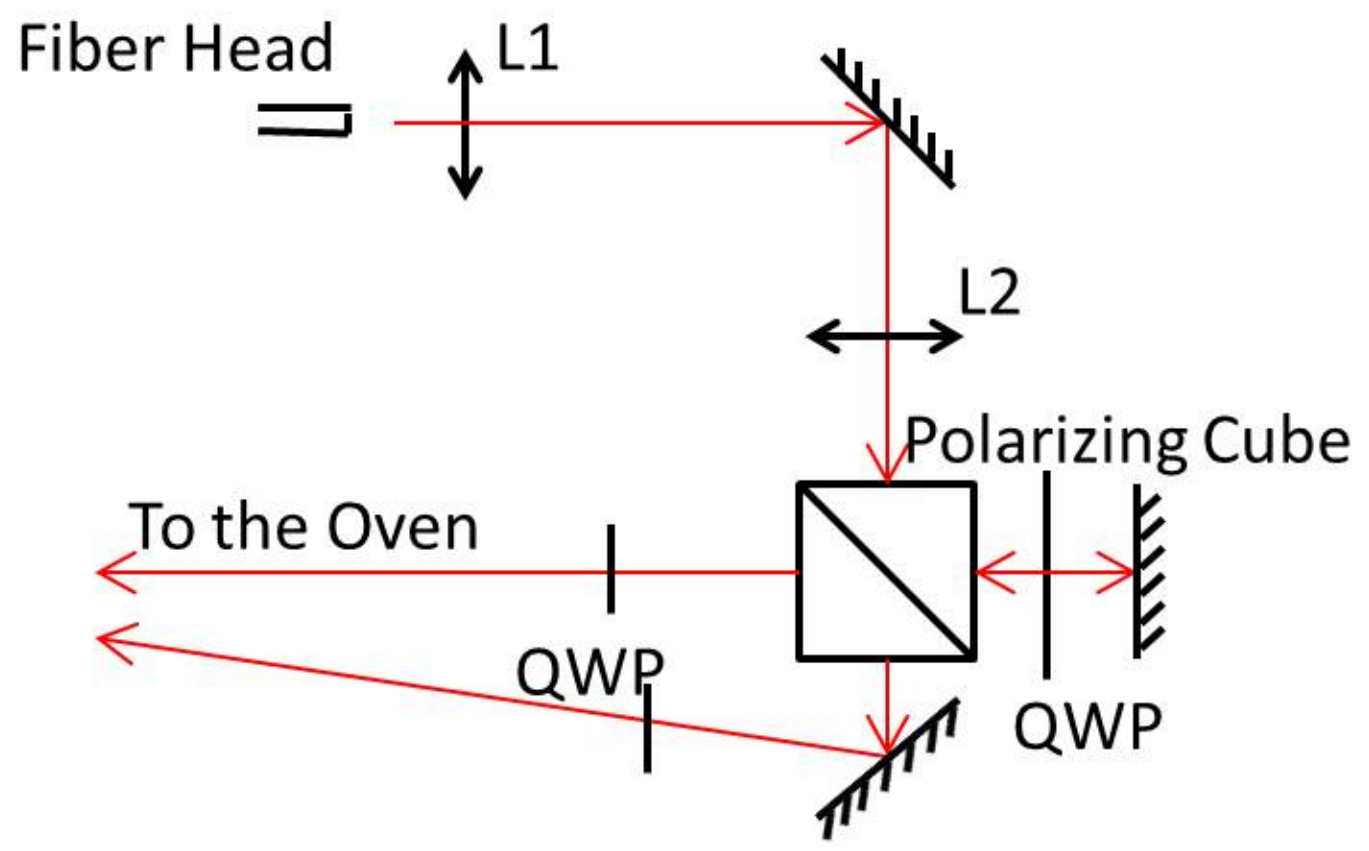

Figure 2.4: Optics for generating circularly polarized laser light for SEOP

the polarizing cube. In the end, the two beams are circularly polarized by two separate quarter wave plates and passed on to the cell.

\subsubsection{Solving for Polarization}

Let's consider the populations of the two ground states, $\rho_{+1 / 2}$ and $\rho_{-1 / 2}$. They are normalized such that $\rho_{+1 / 2}+\rho_{-1 / 2}=1$, and the polarization is defined as $P_{\mathrm{Rb}}=$ $\frac{\rho_{+1 / 2}-\rho_{-1 / 2}}{\rho_{+1 / 2}+\rho_{-1 / 2}}=\rho_{+1 / 2}-\rho_{-1 / 2}$. During optical pumping, the evolution of the populations can be described by

$$
\frac{d \rho_{ \pm 1 / 2}}{d t}= \pm\left[\frac{\Gamma_{R b}}{2}+R_{o p t}\right] \rho_{-1 / 2} \mp \frac{\Gamma_{R b}}{2} \rho_{+1 / 2},
$$

where $\Gamma_{R b}$ is the spin destruction rate, and $R_{o p t}$ is the the optical pumping rate per Rb atom. By solving Eq. 2.4, the Rb polarization can be calculated as

$$
P_{\mathrm{Rb}}(t)=\rho_{+1 / 2}-\rho_{-1 / 2}=\frac{R_{o p t}}{R_{o p t}+\Gamma_{R b}}\left[1-e^{-\left(R_{o p t}+\Gamma_{R b}\right) t}\right],
$$

which shows an exponential build-up of polarization. Since the optical pumping time scale is on the order of a few tens of milliseconds, for our purposes it is a good assump- 
tion to only consider the saturation Rb polarization in SEOP, which is

$$
P_{R b}=\frac{R_{o p t}}{R_{o p t}+\Gamma_{R b}}
$$

Thus a high $\mathrm{Rb}$ polarization can be achieved if the laser pumping rate greatly exceeds the total $\mathrm{Rb}$ spin relaxation rate.

\subsubsection{Optical Pumping Rate}

The optical pumping rate per $\mathrm{Rb}$ atom can be described by

$$
R_{o p t}(\overrightarrow{\mathbf{r}}, \nu)=\int \boldsymbol{\Phi}(\overrightarrow{\mathbf{r}}, \nu) \sigma(\nu) d \nu
$$

where $\boldsymbol{\Phi}(\overrightarrow{\mathbf{r}}, \nu)$ is the position dependent photon flux spectral density, and $\sigma(\nu)$ is the photon absorption cross section at the frequency $\nu$. This cross section can to a good approximation be assumed to have a Lorentzian lineshape with a linewidth $\Gamma$,

$$
\sigma(\nu)=\frac{\sigma_{0}}{1+4\left(\nu-\nu_{0}\right)^{2} / \Gamma^{2}} .
$$

The linewidth is a combination of natural line width $(5.7 \mathrm{MHz})$, Doppler broadening $\left(250 \mathrm{MHz}\right.$ at $\left.200^{\circ} \mathrm{C}\right)$ and pressure broadening [20]. Pressure broadening is caused by collisions of $\mathrm{Rb}$ with other gases in the cell. For cells with total pressure on the order of an amagat, the pressure broadening width dominates. It is also the case that the center of line will be slightly shifted due to such collisions. Table 2.1 shows the coefficients needed to evaluate these effects. The parameters in the second column can be used to determine the linewidth $\Gamma$, and the parameters in the third column can be used to calculate the shift of the of the line center $\nu_{0}$. The temperature dependence of these parameters are given in the last column.

The peak absorption cross section can be calculated from the sum rule

$$
\int \sigma(\nu) d \nu=\pi r_{e} c f
$$

where $r_{e}$ is the classical electron radius and $\mathrm{f}$ is the oscillator strength of the transition. For $\mathrm{Rb} \mathrm{D}_{1}$ line, the oscillator strength $\mathrm{f}=0.337$ [22].

Plugging Eq. 2.8 into Eq. 2.9, we can derive the relationship between the $\sigma_{0}$ and the line width:

$$
\sigma_{0} \Gamma=5.7 \times 10^{-3} \mathrm{~cm}^{2} / \mathrm{s}
$$




\begin{tabular}{cccc}
\hline \hline & $\mathrm{D}_{1}$ full width $(\mathrm{GHz} / \mathrm{amg})$ & $\mathrm{D}_{1}$ line shift $(\mathrm{GHz} / \mathrm{amg})$ & Temp. Depen. \\
\hline${ }^{4} \mathrm{He}$ & $18.0 \pm 0.2$ & $4.3 \pm 0.1$ & $\mathrm{~T}^{0.05 \pm 0.05}$ \\
${ }^{3} \mathrm{He}$ & $18.7 \pm 0.3$ & $5.64 \pm 0.15$ & $\mathrm{~T}^{1.1 \pm 0.1}$ \\
$\mathrm{~N}_{2}$ & $17.8 \pm 0.3$ & $-8.25 \pm 0.15$ & $\mathrm{~T}^{0.3}$ \\
$\mathrm{Xe}$ & $19.2 \pm 2$ & $-6.95 \pm 0.7$ & $\mathrm{~T}^{0.3}$ \\
\hline \hline
\end{tabular}

Table 2.1: Pressure broadening and shift density coefficients for $R b D_{1}$ line by gases commonly involved in SEOP [21]. The coefficients here are measured at $353 \mathrm{~K}$ and can be scaled with the given temperature dependence.

Consider a SEOP cell with 2.5 amagats of gas in total. Since the $\mathrm{D}_{1}$ pressure broadening coefficients are similar for all kinds of gases commonly involved in SEOP, we can assume a $\mathrm{D}_{1}$ line FWHM of $45 \mathrm{GHz}$ without specifying further details of the gas composition. Therefore, the peak amplitude of the absorption line is $\sigma_{0}=1.3 \times$ $10^{-13} \mathrm{~cm}^{2}$.

If we assume the laser beam has Gaussian profiles both in its spatial distribution and spectrum [19], the position dependent photon flux spectral density can be described by

$$
\begin{aligned}
\Phi(\nu, \vec{r}) & =\Phi_{s p}(\vec{r}) G(\nu) \\
\Phi_{s p}(\vec{r}) & =\Phi_{0} e^{-2 r^{2} / w^{2}}=\frac{P}{h \nu} \frac{2}{\pi w^{2}} e^{-2 r^{2} / w^{2}} \\
G(\nu) & =\frac{1}{\sigma_{l} \sqrt{2 \pi}} e^{-\frac{\left(\nu-\nu_{0}\right)^{2}}{2 \sigma_{l}^{2}}}
\end{aligned}
$$

where $\Phi_{0}$ is the photon density at the center of the beam, and it's related to the total laser power $\mathrm{P}$ and beam waist $w$. We can estimate the average photon density within the beam waist range as $\overline{\Phi_{s p}}=\frac{\int_{0}^{w} \Phi_{0} e^{-2 r^{2} / w^{2}} 2 \pi r d r}{\pi w^{2}}=\frac{0.865 P}{h \nu \pi w^{2}}$. For $20 \mathrm{~W}$ laser power with $1.5 \mathrm{~cm}$ beam radius, $\overline{\Phi_{s p}}=9.78 \times 10^{18} \mathrm{~cm}^{-2} . \sigma_{l}$ is the Gaussian width of the laser spectrum, and $\sigma_{l}=\frac{\Gamma_{\text {laser }}}{2 \sqrt{l n 4}}$. For lasers with FWHM of $0.2 \mathrm{~nm}(95 \mathrm{GHz}), \sigma_{l}=40 \mathrm{GHz}$. Now we can estimate the average optical pumping rate at the the front of the cell under the aforementioned conditions using Eq. 2.7, and $\overline{R_{o p t}} \sim 587 \mathrm{kHz}$.

The laser light is attenuated as it propagates through the bulk of the cell [7]:

$$
\frac{\partial \Phi(\nu)}{\partial z}=-[R b]\left(1-P_{R b}\right) \Phi(\nu) \sigma(\nu)
$$

The laser intensity decreases, and the spectrum becomes less favorable for optical pumping as the laser light goes into the cell. Dolph simulated this process and plotted the 
change of light intensity and spectral profile as a function of the depth into the cell in section 2.2.7 of his Ph.D thesis. If the total laser power is not enough, there will be a $\mathrm{Rb}$ polarization gradient in the cell.

\subsubsection{Rb Spin Destruction Rate}

The destruction of $\mathrm{Rb}$ polarization can be divided into two major categories: binary collision and the formation and breakup of van der Waals molecules.

The time scale of binary collisions is on the order of a ps. During such collisions, the $\mathrm{Rb}$ polarization can be lost to the motional degree of freedom of the colliding pair. In ${ }^{3} \mathrm{He}$ cells, the total $\mathrm{Rb}$ spin destruction rate due to binary collisions is:

$$
\left.\Gamma_{\mathrm{Rb}}^{b c}=k_{\mathrm{Rb}-\mathrm{Rb}}[\mathrm{Rb}]+k_{\mathrm{Rb}-{ }^{3} \mathrm{He}}{ }^{3} \mathrm{He}\right]+k_{\mathrm{Rb}-\mathrm{N}_{2}}\left[\mathrm{~N}_{2}\right],
$$

where $k_{\mathrm{Rb}-\mathrm{X}}$ is the spin destruction rate constant due to the collision between $\mathrm{Rb}$ and the gas $\mathrm{X}$ (which can also be $\mathrm{Rb}$ ), and $[\mathrm{X}]$ is the density of that gas. For Xe cells, $k_{\mathrm{Rb}-{ }^{3} \mathrm{He}}\left[{ }^{3} \mathrm{He}\right]$ should be replaced by $k_{\mathrm{Rb}-\mathrm{Xe}}[\mathrm{Xe}]$. Singh and Dolph fit previous measurements of $k_{\mathrm{Rb}-{ }^{3} \mathrm{He}}$ and $k_{\mathrm{Rb}-\mathrm{N}_{2}}$ to a temperature dependent model, and their results are given by [19]:

$$
\begin{aligned}
k_{\mathrm{Rb}-{ }^{3} \mathrm{He}}(T) & =55.9(9)\left(\frac{T}{473.15 K}\right)^{3.31(12)} \mathrm{Hz} / \mathrm{amg} \\
k_{\mathrm{Rb}-\mathrm{N}_{2}}(T) & =290(30)\left(\frac{T}{473.15 K}\right)^{2.00(25)} \mathrm{Hz} / \mathrm{amg}
\end{aligned}
$$

Nielson et. al. 23] measured $k_{\mathrm{Rb}-\mathrm{Xe}}$ to be:

$$
\begin{aligned}
k_{\mathrm{Rb}-\mathrm{Xe}}\left(150^{\circ} C\right) & =2.44 \times 10^{5} \quad \mathrm{~Hz} / \mathrm{amg} \\
k_{\mathrm{Rb}-\mathrm{Xe}}\left(80^{\circ} C\right) & =2.28 \times 10^{5} \quad \mathrm{~Hz} / \mathrm{amg}
\end{aligned}
$$

$k_{\mathrm{Rb}-\mathrm{Rb}}$ is assumed to be temperature independent and has been measured by Kadlecek [24] that at holding fields $\ll 1 \mathrm{kG}$,

$$
k_{\mathrm{Rb}-\mathrm{Rb}}=4.45 \times 10^{-13} \mathrm{~cm}^{3} / \mathrm{s} .
$$

Rb spin destruction due to vdW molecules is only important in cells containing Xe. Nielson et. al. measured this rate in the "short lifetime" region [23] (total gas pressure on the order of an amagat). They reported that with a gas mixture of $1 \% \mathrm{Xe}, 1 \%$ $\mathrm{N}_{2}$ and $98 \% \mathrm{He}$, the vdW spin destruction rate is $2049 \mathrm{~s}^{-1}$ at $150^{\circ} \mathrm{C}$ and $3240 \mathrm{~s}^{-1}$ at 
$80^{\circ} \mathrm{C}$. These rates are independent of the total gas pressure, but dependent on the gas composition. Their results also included the vdW spin exchange rate, which depends on the isotopic abundance of the Xe gas. We'll ignore these details for now and use their result as an estimate for xenon gas in general.

The efficiency of each gas species in the forming and breaking up a $\mathrm{Rb}-\mathrm{Xe} \mathrm{vdW}$ molecule has been studied in Xe nucleus spin exchange experiments [25, 26, 27], and summarized in [28]. Using these coefficients, we can generalize Nielson's result to any gas compositions:

$$
\Gamma_{v d W}(T)=\frac{32.3}{1+0.92 f_{\mathrm{N}_{2}}+0.31 f_{\mathrm{He}}} \Gamma_{N}(T),
$$

where $\Gamma_{N}(T)$ is the spin relaxation rate measured with Nielson's gas composition at temperature T. $f_{\mathrm{N}_{2}}=\left[\mathrm{N}_{2}\right] /[\mathrm{Xe}]$ and $f_{\mathrm{He}}=[\mathrm{He}] /[\mathrm{Xe}]$ are the relative fraction of gases. Ruset extracted a smilar formula in his thesis [29].

Consider the $\mathrm{Rb}$ spin destruction rate in a ${ }^{3} \mathrm{He}$ SEOP cell with 2.5 amagats of ${ }^{3} \mathrm{He}$, 0.15 amagats of $\mathrm{N}_{2}$ at $180^{\circ} \mathrm{C}\left(\sim 4.3 \times 10^{14} \mathrm{~cm}^{-3}\right)$. It is straight forward to calculate that:

$$
\begin{aligned}
\Gamma_{\mathrm{Rb}-{ }^{3} \mathrm{He}}^{b c} & \approx 121 \mathrm{~Hz} \\
\Gamma_{\mathrm{Rb}-\mathrm{N}_{2}}^{b c} & \approx 40 \mathrm{~Hz} \\
\Gamma_{\mathrm{Rb}-\mathrm{Rb}}^{b c} & \approx 191 \mathrm{~Hz} \\
\Gamma_{\mathrm{Rb}}^{\text {Total }} & \approx 352 \mathrm{~Hz}
\end{aligned}
$$

where the vdW interaction is ignored.

For a cell with 2.5 amagats of $\mathrm{Xe}, 0.15$ amagats of $\mathrm{N}_{2}$ at $115^{\circ} \mathrm{C}([\mathrm{Rb}] \sim 1.5 \times$ $10^{13} \mathrm{~cm}^{-3}$ ), we have:

$$
\begin{aligned}
\Gamma_{\mathrm{Rb}-\mathrm{Xe}}^{b c} & \approx 590 \mathrm{kHz} \\
\Gamma_{\mathrm{Rb}-\mathrm{N}_{2}}^{b c} & \approx 29 \mathrm{~Hz} \\
\Gamma_{\mathrm{Rb}-\mathrm{Rb}}^{b c} & \approx 7 \mathrm{~Hz} \\
\Gamma_{\mathrm{Rb}-\mathrm{Xe}}^{v d W} & \approx 81 \mathrm{kHz} \\
\Gamma_{\mathrm{Rb}}^{\text {Total }} & \approx 671 \mathrm{kHz}
\end{aligned}
$$

For a cell with 0.1 amagats of Xe, 0.15 amagats of $\mathrm{N}_{2}$ and 1 amagat of $\mathrm{He}$ (we will use the spin destruction rate for ${ }^{3} \mathrm{He}$ as an estimate below) at $115^{\circ} \mathrm{C}$, we have:

$$
\Gamma_{\mathrm{Rb}-\mathrm{Xe}}^{b c} \approx 23.6 \mathrm{kHz}
$$




$$
\begin{aligned}
\Gamma_{\mathrm{Rb}-\mathrm{N}_{2}}^{b c} & \approx 29 \mathrm{~Hz} \\
\Gamma_{\mathrm{Rb}-\mathrm{He}}^{b c} & \approx 29 \mathrm{~Hz} \\
\Gamma_{\mathrm{Rb}-\mathrm{Rb}}^{b c} & \approx 7 \mathrm{~Hz} \\
\Gamma_{\mathrm{Rb}-\mathrm{Xe}}^{v d W} & \approx 15.6 \mathrm{kHz} \\
\Gamma_{\mathrm{Rb}}^{T o t a l} & \approx 39.2 \mathrm{kHz}
\end{aligned}
$$

In the estimations above, the gas compositions and cell temperatures are chosen so that they resemble the cells that will be discussed in $\mathrm{Ch}$. 3 and 4 . In calculating $\Gamma_{\mathrm{Rb}-\mathrm{Xe}}^{b c}$ and $\Gamma_{\mathrm{Rb}-\mathrm{Xe}}^{v d W}$ at $115^{\circ} \mathrm{C}, k_{\mathrm{Rb}-\mathrm{Xe}}\left(115^{\circ} \mathrm{C}\right)$ and $\Gamma_{N}\left(115^{\circ} \mathrm{C}\right)$ are estimated by linear interpolations of Nielson's results at $80^{\circ} \mathrm{C}$ and $150{ }^{\circ} \mathrm{C}$.

Remember that in the previous section, we worked out that our typical optical pumping rate is about $587 \mathrm{kHz}$, which is much bigger than the $\mathrm{Rb}$ spin destruction rate of a ${ }^{3} \mathrm{He}$ cell, bigger than the spin destruction rate of a low Xe pressure cell, and comparable to that of a high Xe pressure cell. The implication is that it is easy to maintain a close to unity $\mathrm{Rb}$ polarization in a ${ }^{3} \mathrm{He}$ cell. However, in a Xe cell, the Rb polarization strongly depends on the Xe pressure, and it's hard to achieve a high Rb polarization for cells with several amagats of Xe.

\subsubsection{More about Optical Pumping}

In this section we discuss some details about optical pumping that we previously simplified or ignored: the nuclear spin is non-zero; the laser is not fully circularly polarized and has a small skew angle; the cell wall also relaxes Rb polarization.

\subsubsection{Nuclear Slowing Down Factor}

Spin-exchange collisions between pairs of optically pumped $\mathrm{Rb}$ atoms redistribute the total spin angular momentum between $\mathrm{Rb}$ atoms. Within each $\mathrm{Rb}$ atom, the electron and nuclear spins are coupled by the hyperfine interaction, which has little effect during the spin-exchange collisions, but between collisions it transfers angular momentum internally between the nuclear and electron spins. Successive spin-exchange collisions eventually lead to the "spin-temperature" distribution [30]:

$$
\rho_{\mathrm{Rb}}=\frac{e^{\beta_{\mathrm{Rb}} S_{z}}}{Z\left(S, \beta_{\mathrm{Rb}}\right)} \frac{e^{\beta_{\mathrm{Rb}} I_{z}}}{Z\left(I, \beta_{\mathrm{Rb}}\right)},
$$


and $Z(K, \beta)$ is the partition function given by [7]:

$$
Z(K, \beta)=\frac{\sinh [\beta(K+1 / 2)]}{\sinh [\beta / 2]} .
$$

where K can be either I or S. In Eq. 2.37, $\mathbf{S}$ is the electron spin and $\mathbf{I}$ is the nuclear spin. $\rho_{\mathrm{Rb}}$ is the probability that a $\mathrm{Rb}$ atom is in a state with electron spin $\mathrm{S}_{z}$ and nuclear spin $\mathrm{I}_{z}$. The parameter $\beta_{\mathrm{Rb}}$ is proportional to the inverse of spin temperature, which can be used to determine other observables [7]:

$$
<K_{z}>=\frac{\epsilon(K, \beta)}{2} \tanh \frac{\beta}{2}
$$

in which

$$
\epsilon(K, \beta)=2<K(K+1)-K_{z}^{2}>
$$

where $<>$ means ensemble average. In the special case of $\mathbf{K}=\mathbf{S}, \epsilon(K, \beta)=\epsilon(1 / 2, \beta)=$ 1.

The nuclear slowing down factor is defined as the ratio of nuclear and electron angular momentum along the holding field direction [31]:

$$
s l=\frac{<F_{z}>}{<S_{z}>}=1+\frac{<K_{z}>}{<S_{z}>}=1+2<K(K+1)-K_{z}^{2}>,
$$

where $\mathrm{F}$ is the total angular momentum. As the name suggests, the slowing down factor slows down the processes that polarize or relax the Rb polarization. Thus the nuclear

spin can be considered as a reservoir of angular momentum, and buffers the change of $\mathrm{Rb}$ polarization. Therefore it takes longer for the $\mathrm{Rb}$ atoms to reach the saturation polarization, but the saturation polarization is not changed [31],

$$
P_{\mathrm{Rb}}(t)=\frac{R_{o p t}}{R_{o p t}+\Gamma_{S D}}\left[1-e^{\frac{-\left(R_{o p t}+\Gamma_{S D}\right) t}{s l}}\right]
$$

It is worth noting that the slowing-down factor is polarization dependent, and it varies for different isotopes. For natural abundance $\mathrm{Rb}, \mathrm{sl}=10.8$ for $\mathrm{P}=0$ and $\mathrm{sl}=5.4$ for $\mathrm{P}=1$ 31.

\subsubsection{Laser Polarization and Skew Angle}

The laser beam can be imperfect in many ways. Here we consider the effect of less than $100 \%$ circular polarization and a small angle between the laser propagation direction and the holding field direction. Both of the imperfections will induce non-desirable 
transitions in optical pumping, i.e. a small portion of photons excite the $S_{+1 / 2} \rightarrow S_{-1 / 2}$ transition while the majority of photons excite the $S_{-1 / 2} \rightarrow S_{+1 / 2}$ transition. The overall effect is that the $\mathrm{Rb}$ polarization is lower, and more laser power is consumed [32, 33]:

$$
\begin{aligned}
P_{\mathrm{Rb}}(t=\infty) & =\frac{R_{o p t}}{R_{o p t}+\Gamma_{R b}} P_{\text {light }} \cos \theta \\
\frac{\partial \Phi(\nu)}{\partial z} & =-[\mathrm{Rb}]\left(1-P_{\mathrm{Rb}} P_{\text {light }} \cos \theta\right) \Phi(\nu) \sigma(\nu),
\end{aligned}
$$

where $\mathrm{P}_{\text {light }}$ is the circular polarization of the light, and $\theta$ is the skew angle.

\subsubsection{Wall Relaxation}

Diffusion produces a thin layer near the cell walls in which the Rb polarization drops from the bulk polarization value to zero. The walls are usually bare glass or coated with various silicone derivatives. Once a $\mathrm{Rb}$ atom reaches the wall, it stays there for a time long enough that nearly all its nuclear and electron polarizations are lost. The thickness of the polarization transition layer is related to the Rb diffusion and optical

pumping rate, and can be estimated by $\sqrt{D / R}$. For a 10 amagat He cell, this thickness is estimated to be $20 \mu \mathrm{m}$ [5], which is small compared with the cell dimension (usually one to ten centimeters). Therefore this effect is often ignored.

\subsection{Spin Exchange and Relaxation of Noble Gas Nuclei}

Both of the two common SEOP noble gases ${ }^{3} \mathrm{He}$ and ${ }^{129} \mathrm{Xe}$ have a $1 / 2$ nuclear spin, and their nuclear polarization due to spin exchange with polarized Rb atoms can be worked out similarly to the way we solve the Rb polarization in Eq. 2.4. The result is:

$$
P_{n g}(t)=P_{\mathrm{Rb}} \frac{\gamma_{s e}}{\gamma_{s e}+\Gamma}\left[1-e^{-\left(\gamma_{s e}+\Gamma\right) t}\right]
$$

and the saturation nuclear polarization is

$$
P_{n g}(\infty)=P_{\mathrm{Rb}} \frac{\gamma_{s e}}{\gamma_{s e}+\Gamma},
$$

where $\gamma_{s e}$ is the nucleus spin exchange rate with $\mathrm{Rb}$, and $\Gamma$ is the nuclear spin relaxation rate due to all other processes. We'll consider these two mechanisms separately in the next two sections. 


\subsubsection{Spin Exchange Rate}

The noble gas nuclear spin is coupled to the Rb electron spin by an isotropic interaction and an anisotropic interaction. The isotropic interaction aligns the nuclear spin parallel to the electron spin polarization, and the anisotropic interaction polarizes the nuclear spin to the opposite direction. Therefore one interaction has to be much stronger than the other for the noble gas nuclei to be polarized. Fortunately the anisotropic interaction is weak compared with the isotropic interaction, and its effect becomes weaker for heavier noble gases. Walter et.al. estimated in [34 that for ${ }^{3} \mathrm{He}-\mathrm{Rb}$ spin exchange, the anisotropic interaction limits the ${ }^{3} \mathrm{He}$ nuclear polarization to $\sim 95 \%$ of $\mathrm{Rb}$ polarization. For ${ }^{129} \mathrm{Xe}-\mathrm{Rb}$ spin exchange, the limit is about $99 \%$. We'll ignore the anisotropic interaction in the following discussions.

Spin exchange between polarized Rb atoms and the noble gas nuclei occurs either through binary collisions or while the atoms are bound in a van der Waals molecule. The time scale of binary collision is on the order of $\sim 10^{-12} s$ [7], and during a binary collision both $\Delta F= \pm 1$ and $\Delta F=0$ transitions between the hyperfine sublevels of alkali metal atoms can be induced. The contribution of vdW molecules to spin exchange is only important for heavy atoms like Xe. For the total gas pressure of a few tens of torrs to a few amagats (the "short lifetime" region as described in [35]), the life time of the vdW molecules are long enough that only $\Delta F=0$ transitions are induced. At pressures above several amagats, the life time of of the vdW molecules are so short that previous selection rules are broken. Both $\Delta F= \pm 1$ and $\Delta F=0$ transitions are permitted, and the resulting relaxation is nearly indistinguishable from that due to binary collisions [7].

The binary collision is shown is Fig. 2.5(a), It is an important spin exchange mechanism for both ${ }^{3} \mathrm{He}$ and ${ }^{129} \mathrm{Xe}$. The spin exchange rate due to binary collisions is given by:

$$
\gamma_{s e}^{b i}=<\sigma_{s e}^{b i} v>[\mathrm{Rb}]=k_{s e}^{b i}[\mathrm{Rb}],
$$

where $k_{s e}=\left\langle\sigma_{s e} v\right\rangle$ is the velocity-averaged binary spin-exchange rate, which is also

often referred to as the spin exchange rate. These rates have been studied for ${ }^{3} \mathrm{He}[6]$ and ${ }^{129} \mathrm{Xe}$ [36, 37]:

$$
\begin{aligned}
& k_{s e}^{3} \mathrm{He}-\mathrm{Rb}=(6.7 \pm 0.7) \times 10^{-20} \mathrm{~cm}^{3} / \mathrm{s} \\
& k_{s e}^{129} \mathrm{Xe}-\mathrm{Rb}\left(100^{\circ} \mathrm{C}\right)=(2.17 \pm 0.15) \times 10^{-16} \mathrm{~cm}^{3} / \mathrm{s}
\end{aligned}
$$



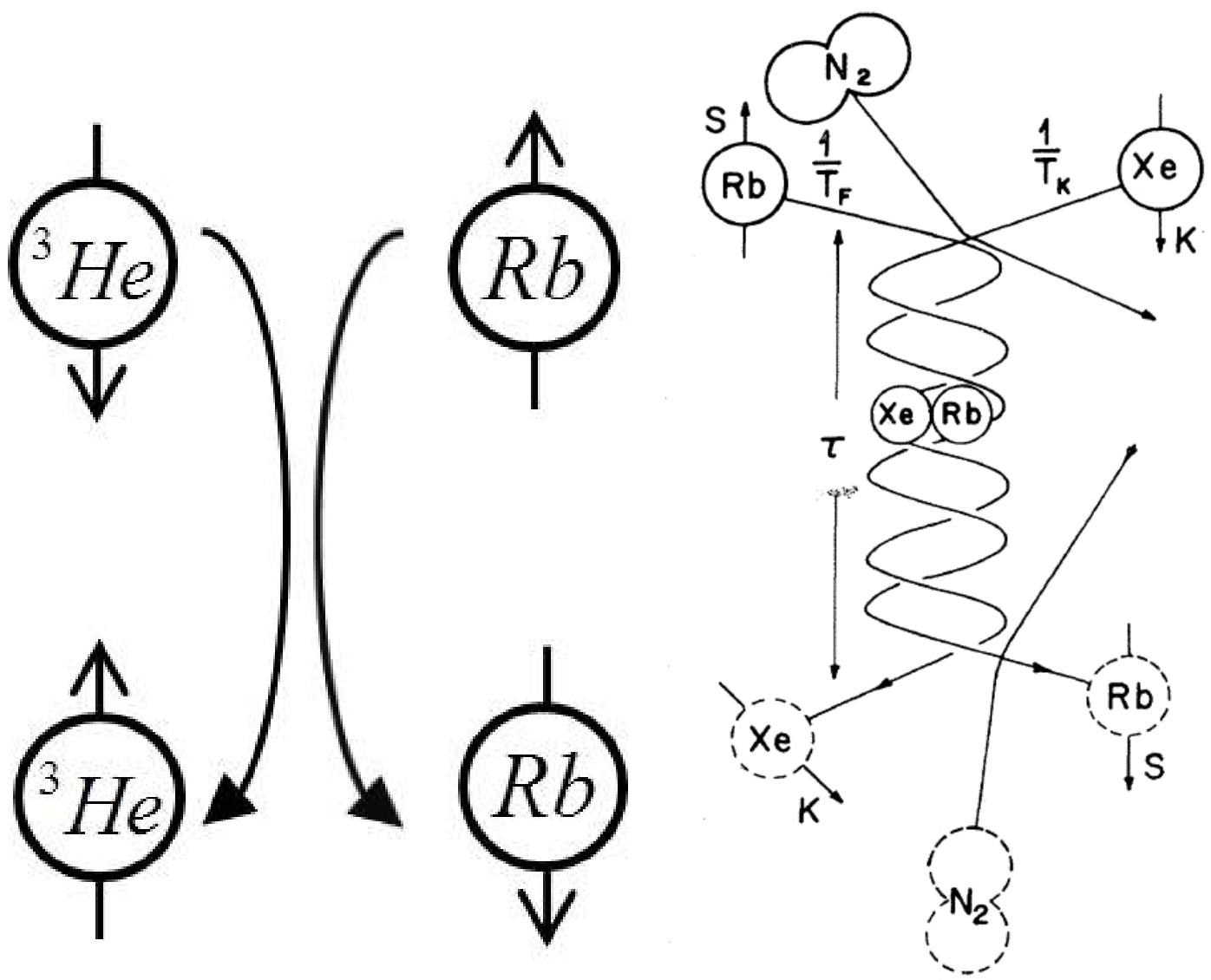

(a) Spin exchange due to binary collisions

(b) Spin exchange through a van der Waals molecule. A third body is needed for the formation and breakup of a vdW molecule. The third body can be $\mathrm{N}_{2}$, Xe, He or other atoms. Adapted from 35]

Figure 2.5: Two Mechanisms for spin exchange between $\mathrm{Rb}$ and noble gas nuclei.

$$
k_{s e}^{129} \mathrm{Xe}-\mathrm{Rb}\left(200^{\circ} \mathrm{C}\right)=(2.23 \pm 0.15) \times 10^{-16} \mathrm{~cm}^{3} / \mathrm{s}
$$

where there is no measurable temperature dependence on $k_{s e}^{3} \mathrm{He}-\mathrm{Rb}$, and a small estimated temperature dependence on $k_{s e}^{129} \mathrm{Xe}-\mathrm{Rb}$.

Spin exchange can also happen by forming a short lived van der Waals molecule, as shown in Fig. 2.5(b), This mechanism is only important for heavy noble gas nuclei like Xe. A third body needs to be involved in this process to satisfy energy and momentum conservation. This third body can be a buffer gas atom/molecule or another Xe atom. 
As Cates et. al. pointed out and measured [26], the spin exchange rate of ${ }^{129} \mathrm{Xe}-\mathrm{Rb}$ due to vdW molecules for a cell with a high Xe pressure (up to a few amagats) is proportional to the inverse of the Xe density. Their formula can be generalized for different gas compositions:

$$
\gamma_{s e}^{v d W}=k_{s e}^{v d W}[\mathrm{Rb}]=\frac{1}{\Sigma\left[\mathrm{M}_{\mathrm{i}}\right] / \kappa_{\mathrm{i}}}[\mathrm{Rb}],
$$

where i stands for a certain gas species, $\left[M_{i}\right]$ is its density, and $\kappa_{i}$ is a coefficient related to the efficiency of this gas in helping forming and breaking vdW molecules. $\kappa_{i}$ for common SEOP gases have been studied [25, 26, 27], and the results are summarized in [28]: $\kappa_{X e}=5230 s^{-1}, \kappa_{N_{2}}=5700 s^{-1}$ and $\kappa_{H e}=17000 s^{-1}$. The temperature dependence is ignored here.

Now we can estimate the spin exchange rates under conditions similar to what we'll discuss in Ch. 3 and 4 . For a ${ }^{3} \mathrm{He}$ cell at $180^{\circ} \mathrm{C}\left([\mathrm{Rb}] \sim 4.3 \times 10^{14} \mathrm{~cm}^{-3}\right)$,

$$
\gamma_{s e}^{t o t}=\gamma_{s e}^{b i}=2.9 \times 10^{-5} s^{-1}=\frac{1}{9.6 \mathrm{hrs}},
$$

and this rate doesn't depend on ${ }^{3} \mathrm{He}$ pressure.

For a cell with 2.5 amagat of $\mathrm{Xe}$ and 0.15 amgat of $\left.\mathrm{N}_{2}\right)$ at $115^{\circ} \mathrm{C}([\mathrm{Rb}] \sim 1.5 \times$ $\left.10^{13} \mathrm{~cm}^{-3}\right)$,

$$
\gamma_{s e}^{t o t}=\gamma_{s e}^{b i}+\gamma_{s e}^{v d W}=3.3 \times 10^{-3} s^{-1}+1.1 \times 10^{-3} s^{-1}=4.4 \times 10^{-3} s^{-1}=\frac{1}{3.8 \mathrm{~min}},
$$

For a cell with 0.1 amagat of $\mathrm{Xe}, 0.15$ amagat of $\mathrm{N}_{2}$ and 1 amagat of $\mathrm{He}$ at $115^{\circ} \mathrm{C}$,

$$
\gamma_{s e}^{t o t}=3.3 \times 10^{-3} s^{-1}+5.4 \times 10^{-3} s^{-1}=8.6 \times 10^{-3} s^{-1}=\frac{1}{1.9 \mathrm{~min}},
$$

For Xe cells with an even lower total pressure, the vdW spin exchange rate can greatly exceed the binary collision spin exchange rate.

\subsubsection{Noble Gas Spin Relaxation Rate}

In general the total noble gas nucleus spin relaxation rate can be described by:

$$
\Gamma=\Gamma_{\text {gas }}+\Gamma_{\text {gradient }}+\Gamma_{\text {wall }}
$$

where the first term is relaxation due to interaction with gases in the cell, the second term is relaxation related to the magnetic field gradient, and the third term is relaxation caused by interactions with the cell wall. When the cell is used as a target for 
electron scattering experiments, there will be another term $\Gamma_{\text {beam }}$ to account for the extra relaxation caused by the electron beam.

For a ${ }^{3} \mathrm{He}$ cell, usually the total amount of other gases is small, and $\Gamma_{\text {gas }}$ is dominated by the ${ }^{3} \mathrm{He}$ dipolar relaxation rate. At room temperature $\left(23^{\circ} \mathrm{C}\right)$, the dipolar relaxation rate is 38 :

$$
\frac{1}{\Gamma_{d i p}}=\frac{\left[{ }^{3} \mathrm{He}\right]}{744} \mathrm{hrs}^{-1}
$$

where $\left[{ }^{3} \mathrm{He}\right]$ is the ${ }^{3} \mathrm{He}$ density in amagats. This relaxation rate has a weak temperature dependence [38]. As the temperature increases from room temperature to $180^{\circ} \mathrm{C}$, it increases by roughly $10 \%$. Therefore the dipolar relaxation rate for a $2.5 \mathrm{amg}$ cell at $180^{\circ} \mathrm{C}$ is about $1 / 271 \mathrm{hs}^{-1}$.

The longitudinal relaxation rate due to diffusion in a magnetic field gradient is [39, 40, 41]:

$$
\Gamma_{\text {gradient }}=D \frac{\left|\nabla B_{x}\right|^{2}+\left|\nabla B_{y}\right|^{2}}{B_{0}^{2}},
$$

where $\mathrm{D}$ is the diffusion coefficient, $\left|\nabla B_{x}\right|$ and $\left|\nabla B_{y}\right|$ are the transverse magnetic field gradients, and $\mathrm{B}_{0}$ is the holding field along $\mathrm{z}$ direction. This formula can be understood in the sense that as a noble gas nucleus moves due to diffusion, the transverse field gradients can be considered as a randomly fluctuating transverse magnetic field, which may induce spin flips if the frequency is right. The self diffusion rate for ${ }^{3} \mathrm{He}$ in a 2.5 amagats ${ }^{3} \mathrm{He}$ cell is about $0.96 \mathrm{~cm}^{2} / \mathrm{s}$ (scaled from [42]). For a perfect Helmholtz coil pair with $66 \mathrm{~cm}$ radius (similar to our holding field coil), the maximum $\left|\nabla B_{t}\right| / B_{0}$ is on the order of $3 \times 10^{-5} \mathrm{~cm}^{-1}$ within $5 \mathrm{~cm}$ from the center of the coil pair. Therefore, the spin relaxation rate due to the magnetic field gradient is no more than $1 /\left(3.2 \times 10^{5} \mathrm{hrs}\right)$.

Another important relaxation mechanism is wall relaxation, which purely depends on the property of the cell inner surface. In our group ${ }^{3} \mathrm{He}$ cells are constructed out of General Electric Type 180 (GE-180) glass, which is nearly impermeable to ${ }^{3} \mathrm{He}$. All the glass tubings were "reblown", a process in which the diameter of the commercial tubings were enlarged using a glass lathe and a hand torch. The "reblown" process has been found to be critical in suppressing the wall relaxation rate [16, 38, 43], perhaps because it fixes microfissures on the interior glass surfaces. An alternative method of suppressing the wall relaxation is to coat the inner surface with sol-gel [44. Although care has been taken to construct cells under similar conditions, the wall relaxation varies a lot between different cells and it's usually the limiting factor for the cell lifetime, although in a few 
cases the dipolar relaxation dominates. The relaxation rates of a series of ${ }^{3} \mathrm{He}$ cells made and characterized by previous students in our lab is summarized in [19].

For ${ }^{129} \mathrm{Xe}$, we also need to take into account the contribution from van der Waals molecules for $\Gamma_{\text {gas }}$. At room temperature, $\Gamma_{\text {gas }}$ in pure Xe is [45]:

$$
\Gamma_{\text {gas }}=6.7 \times 10^{-5} s^{-1}+5.0 \times 10^{-6} s^{-1}[\mathrm{Xe}]
$$

where $[\mathrm{Xe}]$ is the xenon density in amagats. The first term is for the ${ }^{129} \mathrm{Xe}-\mathrm{Xe}$ molecular relaxation in pure Xe, the second term is for binary collision relaxation. For a cell with less than 14 amagats of Xe, the molecular relaxation exceeds the binary collision relaxation. Note that the vdW relaxation rate doesn't rely on the total gas pressure. However, it depends on the gas composition, as suggested in [45],

$$
\Gamma_{v d W}=\frac{6.7 \times 10^{-5} s^{-1}}{1+1.05 f_{1}+0.25 f_{2}},
$$

where $f_{1}=\left[\mathrm{N}_{2}\right] /[\mathrm{Xe}]$ and $f_{2}=[\mathrm{He}] /[\mathrm{Xe}]$ are the relative fractions of $\mathrm{He}$ and $\mathrm{N}_{2}$. The binary collision term should also have contributions from gases other than Xe. However, since Xe has a much bigger spin relaxation cross section with Xe than with other gases, and the binary collision relaxation is already at least several times smaller than the vdW molecule relaxation, we'll ignore these contributions for now. For a cell with 2.5 amagats of xenon and 0.15 amagats of $\mathrm{N}_{2}, \Gamma_{\text {gas }} \sim 7.7 \times 10^{-5} \mathrm{~s}^{-1}=1 / 3.6 \mathrm{hrs}$. For a cell with 0.1 amagats of Xe, 0.15 amagats of $\mathrm{N}_{2}$ and 1 amagats of He, $\Gamma_{\text {gas }} \sim$ $1.7 \times 10^{-5} \mathrm{~s}^{-1}=1 / 16$ hrs.

The gradient relaxation rate for Xe cells is negligible. Since Xe is much heavier than ${ }^{3} \mathrm{He}$, it in general has a much smaller diffusion coefficient which makes the gradient relaxation rate even smaller than that of ${ }^{3} \mathrm{He}$ cells. For example, at $1 \mathrm{~atm}$ and room temperature, Xe self diffusion coefficient is $0.060 \mathrm{~cm}^{2} / \mathrm{s}$ [46].

Now we consider wall relaxation for Xe. Scott Rohrbaugh, a previous member of our group has done extensive work on Xe wall relaxation [47, 48]. A series of 1" diameter Xe cells were made out of Pyrex glass. Usually (except for a control cell) a small amount of $\mathrm{H}_{2}$ or $\mathrm{D}_{2}$ was added into the cell to form a $\mathrm{RbH}$ or $\mathrm{RbD}$ coating on the wall. Xe relaxation rates were measured in a wide magnetic field $(0.08 \mathrm{~T}-1.0 \mathrm{~T})$ and temperature $(205 \mathrm{~K}-323 \mathrm{~K})$ range. At room temperature with $0.08 \mathrm{~T}$ holding field, the cells typically have a lifetime of a few minutes, with the longest one ( $8.3 \mathrm{mins}$ ) observed in a cell with 20 Torr of $\mathrm{H}_{2}$. It is expected that at the typical SEOP field 
( $\sim 20$ Gauss), the lifetime will be a little shorter. The minutes-long lifetime cannot be explained by $\Gamma_{\text {gas }}$ or $\Gamma_{\text {gradient }}$, so the dominant relaxation mechanism of a Xe cell is wall relaxation.

\subsection{SEOP of ${ }^{131 \mathrm{~m}} \mathrm{Xe}$}

Until this point we only discussed SEOP of ${ }^{3} \mathrm{He}$ and ${ }^{129} \mathrm{Xe}$, which were used in the polarimetry study and low field imaging experiments that will be covered in Ch. 3 and 4. However, another Xe isotope, ${ }^{131 \mathrm{~m}} \mathrm{Xe}$, was used in the development of Polarized Nuclear Imaging (PNI) and Polarized Nuclear Detection (PND) that I will introduce in Ch. 5. Polarizing ${ }^{131 \mathrm{~m} X e ~ u s i n g ~ S E O P ~ h a s ~ b e e n ~ r e p o r t e d ~ i n ~ t h e ~ p a s t ~[12, ~ 49], ~ b u t ~}$ details of the SEOP process for this nucleus have not yet been studied. ${ }^{131 \mathrm{~m}} \mathrm{Xe}$ is a spin $11 / 2$ nucleus, and the spin exchange and destruction coefficients can be quite different from ${ }^{129} \mathrm{Xe}$.

SEOP is used as a tool to produce hyperpolarized ${ }^{131 m}$ Xe for our PNI and PND studies. We adjusted SEOP parameters and tried to maximize the ${ }^{131 \mathrm{~m}}$ Xe polarization. I will briefly summarize our results here, and hopefully they will throw some light on future studies on the details of this process.

The dimension of our ${ }^{131 \mathrm{~m}}$ Xe cells is about $2^{\prime \prime}$ across. There are about 0.5 torr of Xe (only a trace amount of which is ${ }^{131 \mathrm{~m}} \mathrm{Xe}$ ), a few torr of $\mathrm{H}_{2}$, and different amount of $\mathrm{N}_{2}$ (on the order of a few hundred torr) in them. The small amount of $\mathrm{H}_{2}$ is supposed to form a $\mathrm{RbH}$ coating on the interior cell wall and suppress relaxation due to quadrupole interactions [50]. These cells have valves on them, so we can freeze Xe in the cell and adjust the amount of $\mathrm{N}_{2}$ conveniently. A Comet Laser and a D-Light laser are used to illuminate the cells 1

In a cell with a total pressure of 150 Torr, we measured the maximum ${ }^{131 \mathrm{~m}} \mathrm{Xe}$ polarization $(67 \%)$ at an oven temperature of $190^{\circ} \mathrm{C}$, and the polarization built up with a time constant of about 25 s. ${ }^{131 \mathrm{~m}}$ Xe polarization dropped quickly below the optimum temperature, and is too small to measure under $125^{\circ} \mathrm{C}$. In comparison, the optimum oven temperature for ${ }^{129} \mathrm{Xe}$ cells that we will discuss in $\mathrm{Ch}$. 3 and $\mathrm{Ch}$. 4 were under $100^{\circ} \mathrm{C}$, and the polarization time constant is also tens of seconds. The fact

\footnotetext{
${ }^{1}$ This batch of cells were made after the PNI and PND studies in Ch. 5 These cells had different geometry and were studied under different SEOP conditions.
} 
that a significantly higher temperature is needed for ${ }^{131 \mathrm{~m}}$ Xe atoms to be polarized and the polarization time constant doesn't become shorter suggests that the spin-exchange coefficient between $\mathrm{Rb}$ and ${ }^{131 \mathrm{~m}} \mathrm{Xe}$ is much smaller than that between $\mathrm{Rb}$ and ${ }^{129} \mathrm{Xe}$. A smaller spin-exchange coefficient is expected since ${ }^{131 \mathrm{~m}}$ Xe has a much larger nuclear spin $(11 / 2)$, and the spin exchange coefficients due to binary collisions is supposed to scale with $1 / \mathrm{I}^{2}$ [51], where $\mathrm{I}$ is the nuclear spin.

We also noticed that ${ }^{131 \mathrm{~m}}$ Xe polarization decreased in cells with higher pressure. In a 800 Torr cell we measured a maximum polarization of $31 \%$, while in a 100 Torr cell the maximum polarization is $72 \%$. We believe the higher polarization was caused by increased spin-exchange rate due to vdW molecules at lower pressure. 


\section{3}

\section{Xe Polarization Studies and Xe Polarimetry}

Producing highly polarized Xe is of great interest to our lab, not only because Xe isotopes are used for the imaging studies discussed in Ch. 4 and 5 , but also for the development of high performance, economical Xe polarizers. In this chapter, I will talk about our study of Xe polarization as a function of Xe pressure and cell temperature, as well as the methods used for Xe polarimetry.

First I will set forth the basics of Nuclear Magnetic Resonance (NMR). I will mainly use the classic approach to describe the behavior of spins in a magnetic field and the ways to manipulate them. I will then talk about measuring HP gas polarization using Electron Paramagnetic Resonance (EPR). EPR polarimetry is often performed at elevated temperatures $\left(>100^{\circ} \mathrm{C}\right)$, which is ideally suited for probing the polarization of noble gases during SEOP. In the end I will present our study of the ${ }^{129}$ Xe polarization as a function of gas composition and cell temperature. We observed that the optimum temperature and peak polarization increased as the Xe pressure dropped. The highest Xe polarization we measured was close to $80 \%$, in a cell with 50 Torr of Xe at around $100^{\circ} \mathrm{C}$. 


\subsection{NMR Techniques}

\subsubsection{Spins in a Magnetic Field}

Just like ${ }^{1} \mathrm{H}$, both ${ }^{3} \mathrm{He}$ and ${ }^{129} \mathrm{Xe}$ are spin-1/2 nuclei. Nuclei with a non-zero spin have a magnetic moment that can be expressed

$$
\vec{\mu}=\gamma \vec{J}=\frac{\gamma}{2 \pi} h \vec{I}
$$

where $\vec{\mu}$ is the magnetic moment, $\gamma$ is the gyromagnetic ratio, $\vec{I}$ is the spin and $\vec{J}$ is the angular momentum, which equals $\hbar \vec{I}$. These parameters for ${ }^{1} \mathrm{H},{ }^{3} \mathrm{He}$ and ${ }^{129} \mathrm{Xe}$ are summarized in Table. 3.1.

\begin{tabular}{cccc}
\hline \hline Species & Spin & $\gamma / 2 \pi$ & $\mu$ \\
\hline${ }^{1} \mathrm{H}$ & $1 / 2$ & 4.2576 & 2.7944 \\
${ }^{3} \mathrm{He}$ & $1 / 2$ & 3.2434 & 2.1287 \\
${ }^{129} \mathrm{Xe}$ & $1 / 2$ & 1.1777 & 0.7730 \\
\hline \hline
\end{tabular}

Table 3.1: Some nuclear species with their spin, gyromagnetic ratio and magnetic moment. The gyromagnetic ratio is given in $\gamma / 2 \pi$, and the unit is $\mathrm{kHz} /$ Gauss. The magnetic moments are in the unit of the nuclear magneton $\left(5.0508 \times 10^{-31} \mathrm{~J} /\right.$ Gauss $)$.

The energy of a magnetic moment in an external field is $E=-\vec{\mu} \cdot \overrightarrow{B_{0}}=-\mu_{z} B_{0}$, for

$\overrightarrow{B_{0}}$ along the $\mathrm{z}$ axis. We know from quantum mechanics that the angular momentum is quantized, so

$$
E=-\mu_{z} B_{0}=-\gamma B_{0} \hbar m_{z},\left(m_{z}=-I, \ldots,+I\right) .
$$

For spin- $1 / 2$ nuclei there are only two possible states for $m_{z}= \pm 1 / 2$, and the energy level separation is $\gamma B_{0} \hbar$. Therefore transitions between the two levels will be induced if there is an RF frequency $\gamma B_{0}$, which is called the Larmor frequency.

\subsubsection{The Rotating Reference Frame}

In this section I will describe the motion of spins in a magnetic field classically. In a magnetic field $\mathrm{B}_{0}$, a torque $\vec{N}$ is exerted on spins with non-zero magnetic moment:

$$
\vec{N}=\vec{\mu} \times \overrightarrow{B_{0}}
$$


which changes the angular momentum of the spins according to $\vec{N}=\frac{d \vec{J}}{d t}=\frac{1}{\gamma} \frac{d \vec{\mu}}{d t}$. Therefore the evolution of the spin magnetic moment is described by:

$$
\frac{d \vec{\mu}}{d t}=\gamma \vec{\mu} \times \overrightarrow{B_{0}}
$$

The solution of Eq. 3.4 is precession, which means the spins will rotate around the external magnetic field, as shown in Fig. 3.1. The rotating frequency is $\vec{\omega}=-\gamma \overrightarrow{B_{0}}$, which is often referred to as the Larmor frequency.

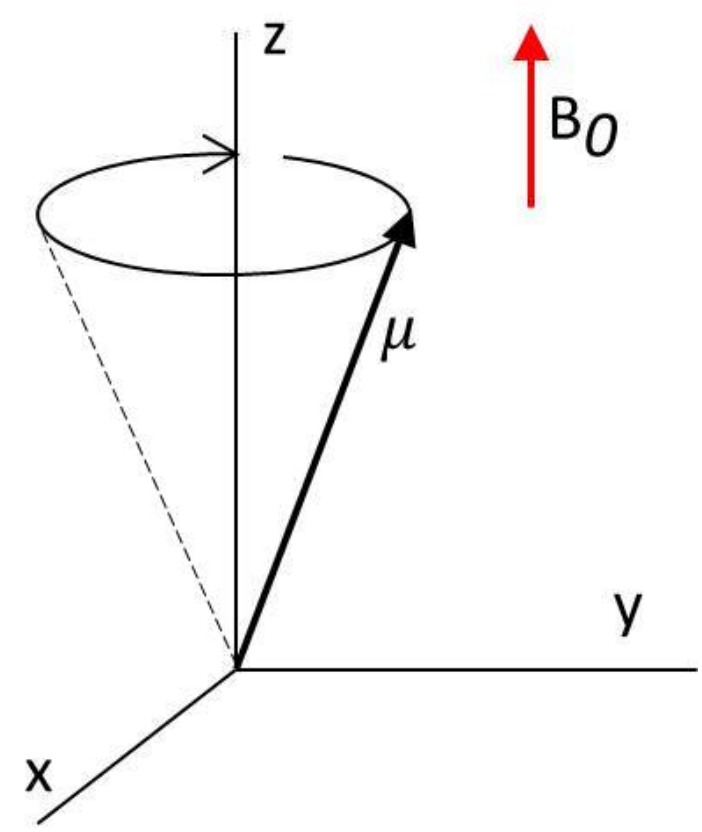

Figure 3.1: Precession of a spin around a holding magnetic field

Now let's consider the spin motion in a frame that rotates at frequency $\vec{\omega}$. Since this frame rotates together with the spin, the spins are stationary in such a frame. We would thus expect the effective magnetic field in this frame to be zero, as was pointed out more rigorously by Rabi, Ramsey and Schwinger in [52]. A fictitious magnetic field is introduced in the rotating frame as $\overrightarrow{B_{f i c}}=\frac{\vec{\omega}}{\gamma}$, where $\omega$ is its rotating frequency. When this frequency exactly matches the Larmor frequency, the effective field in this frame is:

$$
\overrightarrow{B_{e f f}}=\overrightarrow{B_{0}}+\vec{\omega} / \gamma=\overrightarrow{B_{0}}+(-) \gamma \overrightarrow{B_{0}} / \gamma=0 .
$$




\subsubsection{Adiabatic Fast Passage}

The technique of Adiabatic FAst Passage (AFP) technique is a powerful tool for manipulating the magnetization in the presence of RF magnetic field inhomogeneities. In an AFP measurement [53] the spins are tipped 180 degrees with respect to the holding magnetic field, and an electromagnetic signal is induced in the detection coil during the flip. An RF frequency needs to be applied perpendicular to the field, and the flip can be achieved by either sweeping the RF frequency through the resonance frequency defined by $\gamma B_{0}$, or by sweeping the holding field through the resonance field $\left(\omega_{0} / \gamma\right)$ at a fixed RF frequency $\omega_{0}$.

In our lab, we typically sweep the holding field from low value to high value and then sweep back while applying a fixed linearly oscillating RF frequency (though in EPR polarimetry we flip the spins by sweeping the RF frequency). Note that the "down sweep" flips the spins by $180^{\circ}$ again so that they are left in the original direction. The RF frequency can be decomposed into two circularly oscillating components with the same amplitude $\mathrm{B}_{1}$. One component rotates in the same direction as the spin precession and is important in NMR, while the other rotates in the opposite direction and can be ignored. In the frame $\left(x^{\prime}-y^{\prime}-z\right)$ that rotates at the RF frequency $\omega_{0}$, the effective field felt by the spins is:

$$
\overrightarrow{B_{e f f}(t)}=\left(B_{0}(t)-\frac{\omega_{0}}{\gamma}\right) \hat{z}+B_{1} \hat{x}^{\prime}
$$

The coordinates of the rotating frame is defined such that the $\mathrm{B}_{1}$ field is along the $x^{\prime}$ direction. Fig. 3.2 shows the effective holding field during a field sweep. The effective holding field direction changes from $\hat{z}$ to $-\hat{z}$, and it is purely transverse when the larmor frequency matches the RF frequency.

AFP conditions need to be satisfied for a successful NMR measurement:

$$
D \frac{\left|\nabla B_{z}\right|^{2}}{B_{1}^{2}} \ll \frac{\dot{B_{0}}}{B_{1}} \ll \omega_{1},
$$

where $\mathrm{D}$ is the noble gas diffusion coefficient, $\nabla B_{z}$ is the gradient of the holding field, and $\omega_{1}=\gamma B_{1}$ is the precession frequency due to $B_{1}$ field. The $\ll$ on the right side insures that the $\mathrm{B}_{\text {eff }}$ changes direction slowly enough so that the spins can follow it. The $\ll$ on the left side insures that the field sweep rate is much faster than the spin relaxation rate due to transverse field so that the loss is small. 


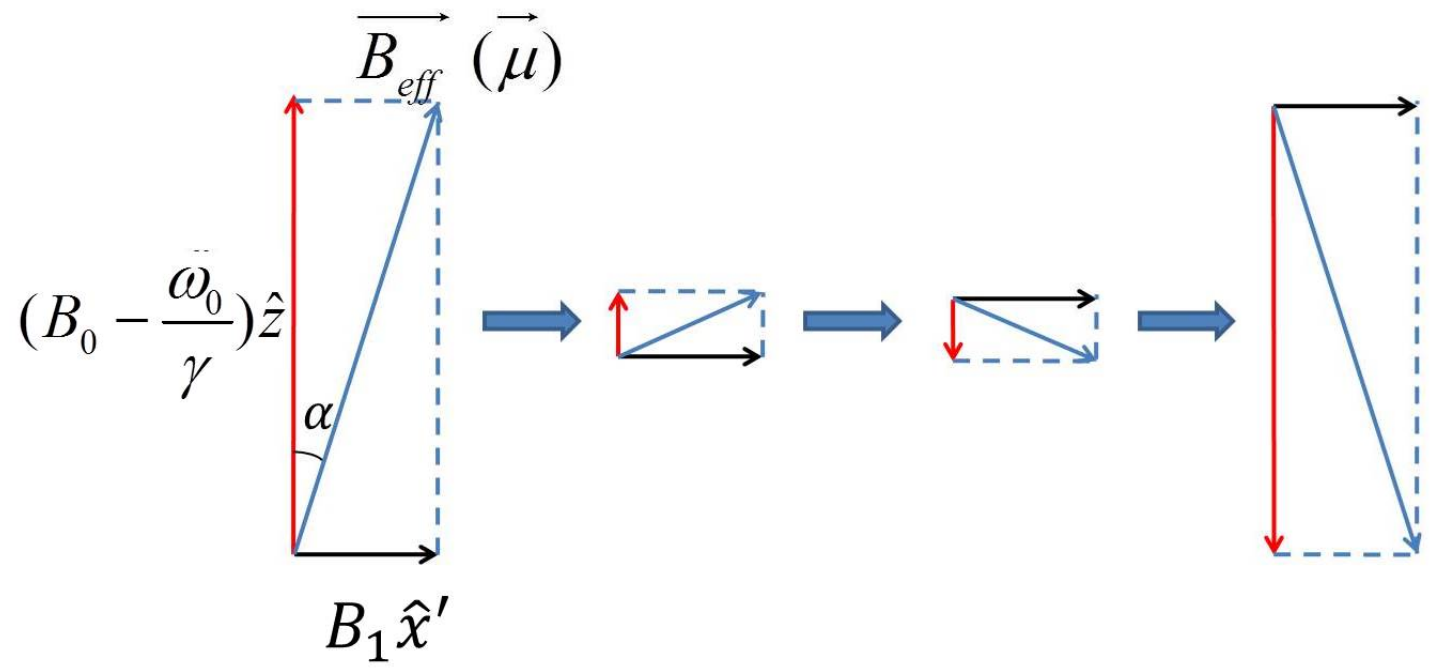

Figure 3.2: Evolution of the effective field in the rotating frame during an AFP measurement. Spins follow the effective field if the condition $\frac{\dot{B}}{B_{1}} \ll \omega_{1}$ is satisfied.

Under our typical operating conditions,

$$
\begin{aligned}
\dot{B}_{0} & \approx 9 \text { Gauss } / 6 \mathrm{~s} \\
B_{1} & \approx 100 \mathrm{mG} \\
\omega_{1} & \approx 320 \mathrm{~Hz} \\
\nabla B_{z} & <5 \mathrm{mGauss} / \mathrm{cm} \\
D & =1.9 \mathrm{~cm}^{2} / \mathrm{s}
\end{aligned}
$$

where $\nabla B_{z}$ and D are estimated for a 2.5" diameter, 1 atm ${ }^{3} \mathrm{He}$ cell. Both AFP conditions are well satisfied under these conditions. For smaller cells with higher pressures (or Xe instead of ${ }^{3} \mathrm{He}$ ), these conditions are better satisfied.

The detection coil is placed near the cell and orthogonal to both the holding field and the RF field. Since the spins are precessing along the holding field while being flipped, an EMF will be induced in the detection cell by the transverse spin magnetization:

$$
S=C \omega \sin \alpha(t)=C \omega \frac{B_{1}}{\sqrt{B_{1}^{2}+\left(B_{0}(t)-B_{1} / \gamma\right)^{2}}},
$$

where $\mathrm{C}$ is a constant to account for the total magnetization, cell and detection coil geometry, and the gain of the detection circuit and electronics, $\omega$ is the precession frequency ( $\mathrm{RF}$ frequency) of the magnetization and $\alpha$ is the angle between the effective 
field and the holding field direction, which is a function of time. Fig. 3.3 shows the signal from an AFP measurement.

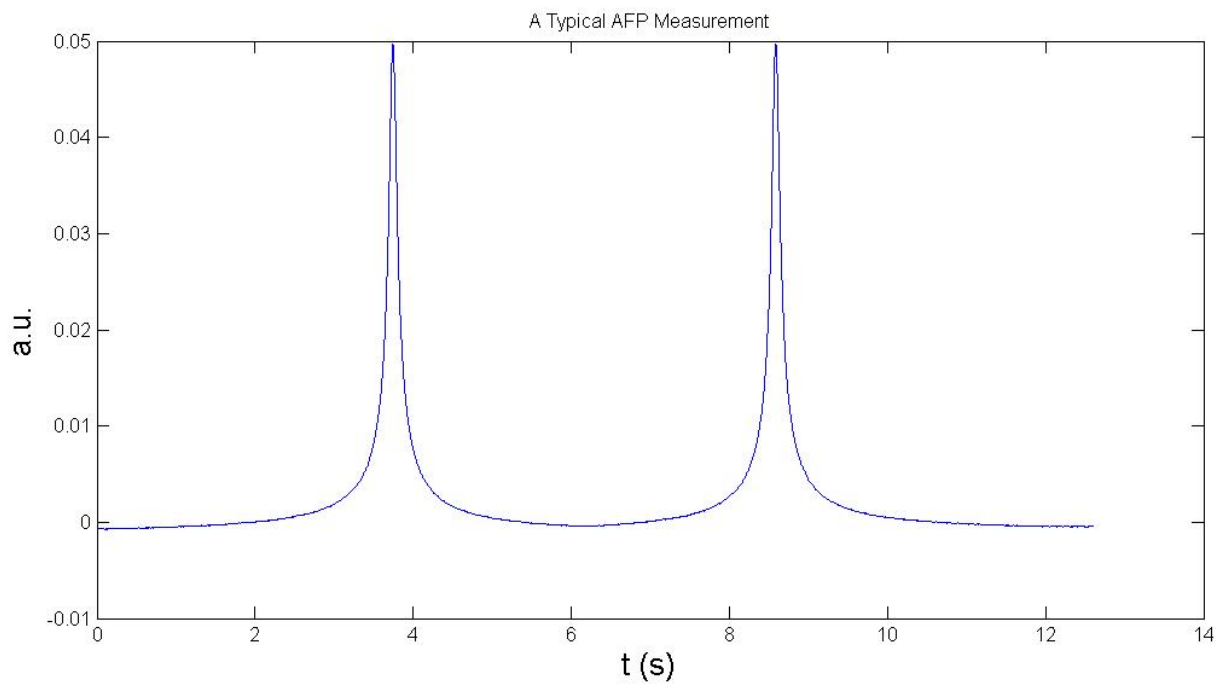

Figure 3.3: A typical AFP measurement in our lab. y axis is the signal amplitude in some arbitrary unit.

\subsubsection{Pulsed NMR}

Pulsed NMR is another technique widely used in NMR. It is in fact by far more common than AFP, and is used, among other things, for MRI.

Unlike AFP measurements, only a short pulse of RF frequency is applied, and the holding field is fixed. The RF frequency is typically on resonance at the Larmor frequency. Thus in the rotating frame, the effective field does not have any components along the holding field $(\mathrm{z})$ direction, but there is a static transverse component due to one of the circularly oscillating components of the RF field. Let's call this field $\mathrm{B}_{1}$ and put it on the $x^{\prime}$ axis in the rotating frame as before. The spins will rotate around the $x^{\prime}$ axis, and be tipped away from $\mathrm{z}$ direction towards $y^{\prime}$ direction by an angle

$$
\alpha=\gamma B_{1} \Delta t
$$

where $\Delta t$ is the RF pulse duration, and $\alpha$ is often referred to as the tip angle.

After spins are tipped by the short RF pulse, there will in general be a transverse component of the magnetization proportional to $\sin \alpha$. This component will then pre- 
cess along the holding field, and a signal will be induced in the transverse detection coil:

$$
s(t)=C \omega_{0} \sin \alpha e^{i\left(\omega_{0} t+\phi\right)},
$$

where $\mathrm{C}$ is a constant, $\omega_{0}$ is the Larmor frequency and $\phi$ is the starting phase. The RF pulse and spin behavior during a pulsed NMR measurement is shown in Fig. 3.4.



Figure 3.4: Schematic of a Pulsed NMR measurement.

Eq. 3.4 doesn't take into account various relaxation mechanism in a pulsed NMR measurement. When a magnetization $\vec{M}$ is not at its equilibrium value, it tends to relax back. The exact time evolution of $\vec{M}$ is described by the Bloch equation [54]:

$$
\frac{d \vec{M}}{d t}=\gamma \vec{M} \times \vec{B}_{0}+\frac{1}{T_{1}}\left(M_{0}-M_{z}\right) \hat{z}-\frac{1}{T_{2}^{*}}\left(M_{x} \hat{x}+M_{y} \hat{y}\right)
$$

where the first term describes precession, and the other two terms describe the relaxation of longitudinal and transverse magnetization separately. $M_{0}$ is the equilibrium magnetization, $1 / T_{1}$ is called the spin-lattice relaxation rate. and it describes the rate at which the longitudinal magnetization returns to the equilibrium value. Physically, $\mathrm{T}_{1}$ involves the exchange of energy between the nuclei and its surroundings. The lowest 
energy state is reached when $\mathrm{M}_{z}$ returns to $\mathrm{M}_{0}$. The transverse relaxation rate can be divided into two parts:

$$
\frac{1}{T_{2}^{*}}=\frac{1}{T_{2}}+\frac{1}{T_{2}^{\prime}}
$$

$\frac{1}{T_{2}}$ is the spin-spin relaxation rate, which is due to the zero-frequency component of the holding field fluctuation caused by the random walk of surrounding dipoles. $\frac{1}{T_{2}^{\prime}}$ is another dephasing mechanism caused by the holding field inhomogeneities, and it usually dominates over $\frac{1}{T_{1}}$ and $\frac{1}{T_{2}}$ relaxation in our experiments. These relaxation mechanisms cause the transverse magnetization to decay while precessing, and the induced signal is called a Free Induction Decay (FID), which can be described by:

$$
s(t)=C \omega_{0} \sin \alpha e^{i\left(\omega_{0} t+\phi\right)} e^{-t / T_{2}^{*}}
$$

where $\mathrm{T}_{2}^{*}$ is dominated by the holding field inhomogeneity. In our setup, $\mathrm{T}_{2}^{*}$ varies between several tens of milliseconds to around 10 seconds, depending on the holding field strength and whether we shim the magnetic field to achieve better homogeneity. We'll talk more about field shimming in Ch. 4.

\subsection{Electron Paramagnetic Resonance Polarimetry}

An important polarimetry technique is to measure the shift of alkali metal Electron Paramagnetic Resonance (EPR) frequency caused by the change of polarized noble gas nuclei magnetization [55]. EPR polarimetry is a non-destructive technique for measuring noble gas polarization, and it's ideally suited for in-situ measurement of the noble gas polarization during SEOP.

When alkali metal atoms are placed in a weak holding field, $\mathrm{F}=\mathrm{J} \pm \mathrm{I}$ is a good quantum number and the states of alkali metal atoms can be described by $\mathrm{F}$ and $\mathrm{m}_{F}$ (Fig. 2.2). The Zeeman transition frequencies between adjacent $\mathrm{m}_{F}$ levels depend on the total effective magnetic field, including the external field $\mathrm{B}_{0}$ and the magnetic field produced by the polarized noble gas nuclei. There is an important enhancement factor for the field induced by noble gas nuclei, which we'll discuss later. Let's take ${ }^{87} \mathrm{Rb}$ for example (Fig. 2.2). Assuming the laser photons used for SEOP carry an angular momentum of +1 , most electrons will thus end up in the $5 \mathrm{~S}_{1 / 2}$ state with $\mathrm{F}=2$ and $\mathrm{m}_{F}=+2$, and can not absorb photons. If an RF frequency corresponding to the $m_{F}=+2 \longrightarrow m_{F}=+1$ transition within the same $\mathrm{F}$ manifold is applied, some 
electrons will be moved out of the $\mathrm{m}_{F}=2$ level and stay in the $\mathrm{m}_{F}=1$ level, in which they can absorb photons and be excited to the $5 \mathrm{P}_{1 / 2}$ state. Most electrons excited to the $5 \mathrm{P}_{1 / 2}$ state are quenched to the ground state by $\mathrm{N}_{2}$. However, a small fraction (usually a few percent for cells in our lab) still decays by emitting a fluorescence photon at either $\mathrm{D}_{1}$ line or $\mathrm{D}_{2}$ line frequency. $\mathrm{D}_{2}$ photons are detected by a photodiode placed behind a $\mathrm{D}_{2}$ filter. Since the intensity of $\mathrm{D}_{2}$ fluorescence is proportional to the fraction of electrons that can be excited to the $5 \mathrm{P}_{1 / 2}$ state, the maximum fluorescence signal is detected when the RF frequency is on resonance for the $m_{F}=+2 \longrightarrow m_{F}=+1$ Zeeman transition. The RF is locked to a frequency corresponding to the maximum $\mathrm{D}_{2}$ light intensity using the methods described in Sec. 3.3.3.1. The locked RF frequency thus indicates the Zeeman transition frequency, and can be used to determine the total effective field.

Zeeman levels are described by the Breit-Rabi equation [56]. In the special case of $\mathrm{L}=0, \mathrm{~S}=1 / 2, \mathrm{~J}=1 / 2$ for $\mathrm{SEOP}$, it can be written as:

$$
\begin{aligned}
E\left(F, m_{F}\right) & =-\frac{h \nu_{h f s}}{2[I]}-g_{I} \mu_{N} B m_{F} \pm \frac{h \nu_{h f s}}{2} \sqrt{1+\frac{4 m_{F}}{[I]} x+x^{2}} \\
\text { with } \quad x & =\left(g_{I} \mu_{N}-g_{s} \mu_{B}\right) \frac{B}{h \nu_{h f s}}
\end{aligned}
$$

where \pm refers to states in the $F=I \pm 1 / 2$ manifold, $\nu_{h f s}$ is the hyperfine splitting frequency, I is the nuclear spin, $[I] \equiv 2 I+1, g_{I}$ and $g_{s}$ are the $g$ factors for nuclear and electron spin, and $\mu_{N}$ and $\mu_{B}$ are the corresponding nuclear and Bohr magneton. Here $\mathrm{x}$ is a unitless factor that gives a relative measure of the size of Zeeman interaction with respect to the size of hyperfine interaction. The transition frequency from $\left(F, m_{F}\right)$ to $\left(F, m_{F}-1\right)$ in the upper manifold is

$$
\begin{aligned}
\nu_{m_{F} \rightarrow m_{F}-1}= & \frac{E\left(F, m_{F}\right)-E\left(F, m_{F-1}\right)}{h} \\
= & -\frac{g_{I} \mu_{N} B}{h} \\
& +\frac{h \nu_{h f s}}{2}\left(\sqrt{1+\frac{4 m_{F}}{[I]} x+x^{2}}-\sqrt{1+\frac{4\left(m_{F}-1\right)}{[I]} x+x^{2}}\right) .
\end{aligned}
$$

The first term has an obvious field dependence, while the field dependence of the second term is hidden in $\mathrm{x}$. Luckily, for our typicle SEOP magnetic field ( $\sim 13$ Gauss $)$, $\mathrm{x}$ is a 
small number and we can Taylor expand Eq. 3.21. The result is:

$$
\begin{aligned}
\nu_{m_{F} \rightarrow m_{F}-1}= & -\frac{g_{I} \mu_{N} B}{h} \\
& +\frac{h \nu_{h f s}}{2}\left(\frac{2 x}{[I]}-\frac{2(2 m-1) x^{2}}{[I]^{2}}+\frac{\left(4-[I]^{2}-12 m+12 m^{2}\right) x^{3}}{[I]^{3}}+\cdots\right) \\
\approx & -\frac{g_{s} \mu_{B}}{h[I]} B \\
& +\frac{g_{s} \mu_{B} B}{2 h[I]}\left(\frac{2(2 m-1) x}{[I]}-\frac{\left(4-[I]^{2}-12 m+12 m^{2}\right) x^{2}}{[I]^{2}}+\cdots\right),
\end{aligned}
$$

where we have used the fact that $\mu_{N} \ll \mu_{B}$ in the simplification. The first term contains the linear dependence of the Zeeman transition frequency on $\mathrm{B}$, and other terms have higher order contributions. Therefore, to the lowest-order approximation, the transition frequency changes linearly with the magnetic field by $\Delta \nu=-\frac{g_{s} \mu_{B}}{h[I]} \Delta B$.

In an EPR measurement, the alkali metal atoms not only experience an external holding field $\mathrm{B}_{0}$, but also feel a small magnetic field caused by the polarized noble gas nuclei. Usually our cells are spherical in shape. The magnetic field inside a uniformly magnetized sphere is [57]:

$$
\vec{B}=\frac{2}{3} \mu_{0} \vec{M}
$$

where $\mu_{0}$ is the vacuum permeability, and $\vec{M}$ is the magnetization. In a hyperpolarized noble gas sample,

$$
\vec{M}=\vec{\mu}_{K} \rho P,
$$

where $\mu_{K}$ is the magnetic moment of the noble gas nucleus, $\rho$ is the noble gas density and $\mathrm{P}$ is its nuclear polarization. However, there is an enhancement to the Fermicontact interaction between the alkali electron and noble gas nucleus. As a result, the field felt by alkali metal atoms due to the noble gas nuclei is:

$$
\Delta \vec{B}=\frac{2}{3} \kappa_{0} \mu_{0} \vec{\mu}_{K} \rho P
$$

where $\kappa_{0}$ is the enhancement factor. $\kappa_{0}$ for $\mathrm{Rb}-{ }^{3} \mathrm{He}$ pairs has been measured accurately by Romalis et. al. in 1998 [55]:

$$
\kappa_{0}^{\mathrm{Rb}-{ }^{3} \mathrm{He}}=4.52+0.00934\left[T\left({ }^{\circ} \mathrm{C}\right)\right]
$$

and the error is $1.5 \%$. Babcock et. al. measured alkali metal- ${ }^{3} \mathrm{He} \kappa_{0}$ again in 2005 and reported very similar result [58]. 
Ma et.al. recently measured the enhancement factor for $\mathrm{Rb}-{ }^{129} \mathrm{Xe}$ pairs [59]. In cells with low Xe density ( $[\mathrm{Xe}] \leq 10$ Torr), $\kappa_{0}^{\mathrm{Rb}-{ }^{129} \mathrm{Xe}}=493 \pm 31$ and this value seems to be independent of temperature. In cells with a Xe pressure roughly 10 times greater, they observed an anomalous drop $(\sim 20 \%)$ at above $160^{\circ} \mathrm{C}$.

Using Eq. 3.22 and Eq. 3.25 , we can relate the noble gas polarization to the change of Zeeman transition frequency, and calculate the polarization if we know the gas density. For a ${ }^{3} \mathrm{He}$ cell at $200^{\circ} \mathrm{C}$, we have

$$
P \approx 0.1388 \frac{\text { Amagat }}{\mathrm{kHz}} \frac{\Delta \nu}{\left[{ }^{3} \mathrm{He}\right]},
$$

where $\mathrm{P}$ is the ${ }^{3} \mathrm{He}$ polarization in percent. We only kept the lowest order term in Eq. 3.27 to give readers a feeling for calculating noble gas polarization from the EPR frequency shift. We actually included higher order terms in our data analysis, which contribute to about $2 \%$ of the transition frequency in a 13 Gauss holding field [19].

\subsection{Xe-129 Polarization at Different Pressures and Tem- peratures}

\subsubsection{Cell Preparation}

We constructed a series of four cells to study the achievable Xe polarization in our lab with different Xe pressures. These cells were roughly 2" diameter spherical cells. They were made of Pyrex glass by an experienced scientific glassblower from Princeton University.

When arriving at our lab, the four cells were attached to a glass manifold, or "string". One end of the string was connected to a bellows. The other end of the string had a glass retort attached to it, which we used to hold an alkali-metal ampoule. The string and cells are shown in Fig. 3.5. There was a $1 / 2$ " vacuum coupling radiation (VCR) connector at the end of the bellows, by which we connected the string to our gas system. We then pumped on the string, first with a mechanical pump then with a diffusion pump. When the pressure dropped to $\sim 10^{-7}$ Torr, we sprayed some helium at joint sections of the string to check if there were any leaks. After making sure there were no leaks on the system, we stopped pumping and back filled the string with clean $\mathrm{N}_{2}$ (purity: 99.999\%). We then opened the retort. A $1 \mathrm{~g}$ ampoule of $\mathrm{Rb}$ was opened 




Figure 3.5: A glass string with 4 cells.

immediately and put in the retort. The retort was then resealed. After that we pumped on the string again with the diffusion pump. We enclosed the string and cells by a big "baking oven", and kept the temperature at $\sim 150^{\circ} \mathrm{C}$ for a few days. For the two ends of the string that couldn't be placed inside the baking oven, we heated them slightly with a flame two or three times a day. This whole baking process was intended to drive off surface contaminants.

After several days of baking, we distilled Rb from the ampoule into a small dip ("Rb reservoir") on the string, hoping to leave behind contaminants from the Rb ampoule in the retort. The retort was then pulled off. The cells were filled one by one. First, we stopped pumping and chased one or two drops of Rb into each cell. Second, we sent the desired amount of Xe into the string, and submerged one of the cells into $\mathrm{LN}_{2}$. At $\mathrm{LN}_{2}$ temperature all Xe would freeze on the cell inner surface and the string pressure would drop to near zero. Third, while keeping the cell in $\mathrm{LN}_{2}$, we sent the right amount of He- $\mathrm{N}_{2}$ gas mixture into the string. The gas mixture was prepared earlier in a 1 L cylindrical volume (the "Calibrated Volume") in the gas system, and was allowed to mix for more than 1 hour. We aimed at $\sim 1000$ Torr total gas pressure at room temperature, so the string pressure with the cell cooled in $\mathrm{LN}_{2}$ was much less than an atmosphere. In the end, we applied a flame on the connection between the cell 
and the string. The connection was melted and the cell was pulled off the string while the pressure difference helped to seal the connection. Only then did we remove the $\mathrm{LN}_{2}$ and bring the cell to room temperature. The rest cells on the string were pulled off similarly.

We also measured the cell volumes during the cell filling procedure. There was a known volume in our gas system that we refer to as the "Calibrated Volume", and we can measure the gas pressure. Before introducing Xe into the string, we measured the total volume of the string and all cells as follows:

1. Rough out the string+cells.

2. Send some clean $\mathrm{N}_{2}$ into the calibrated volume $\left(\mathrm{V}_{0}\right)$ and measure its pressure, $\left(\mathrm{p}_{0}\right)$.

3. Open the calibrated volume to the string, measure the equilibrium pressure, $\left(\mathrm{p}_{1}\right)$.

Assuming the same temperature everywhere, we had $V_{1}=\frac{p_{0} V 0}{p_{1}}-V_{0}$, where $\mathrm{V}_{1}$ was the total volume of the string and cells. After pulling off a cell, we repeated the measurement, but this time the volume measured was $V_{2}=V_{1}-V_{\text {cell }}$. Therefore the cell volume was the difference between $\mathrm{V}_{1}$ and $\mathrm{V}_{2}$.

The natural isotopic abundance of ${ }^{129} \mathrm{Xe}$ is $26.4 \%$. In our experiment we used isotopically enriched Xe with $86.2 \%{ }^{129} \mathrm{Xe}$. The advantage is that with the same Xe pressure, there are more nuclei that can be polarized and contribute to the signal size. The total pressure of each cell was $\sim 1000$ Torr, with the balance of the mixture being mostly ${ }^{4} \mathrm{He}$ and some $\mathrm{N}_{2}$. The total pressure 1000 Torr provided pressure broadening of the Rb absorption line which helped to absorb photons from our spectrally-narrowed lasers.

Table. 3.2 summarizes the volumes and contents of the four cells.

\subsubsection{SEOP of the Cells}

Three Comet lasers were used for this study, and the total laser power was close to 50 W. With about 1000 Torr total gas pressure in the cells, the Rb D1 absorption lines were relatively narrow (FWHM $22.5 \mathrm{GHz})$. The FWHM of Comet lasers were about $\sim 95 \mathrm{GHz}$. It was thus important to center the laser spectra at the Rb D1 transition frequency. 


\begin{tabular}{cccccc}
\hline \hline Cell Name & Volume(cc) & Xe(Torr) & He(Torr) & $\mathrm{N}_{2}$ (Torr) & Total(Torr) \\
\hline $\mathrm{X} 1$ & 50.2 & 56.6 & 818.8 & 131.0 & 1006.4 \\
\hline $\mathrm{X} 2$ & 50.5 & 101.9 & 712.5 & 179.0 & 993.4 \\
\hline $\mathrm{X} 3$ & 53.1 & 144.1 & 640.9 & 196.0 & 981.1 \\
\hline $\mathrm{X} 4$ & 50.7 & 201.1 & 552.4 & 238.4 & 991.9 \\
\hline \hline
\end{tabular}

Table 3.2: 2" Xe Cells X1-X4 volumes and contents

The pressure was measured at room temperature.

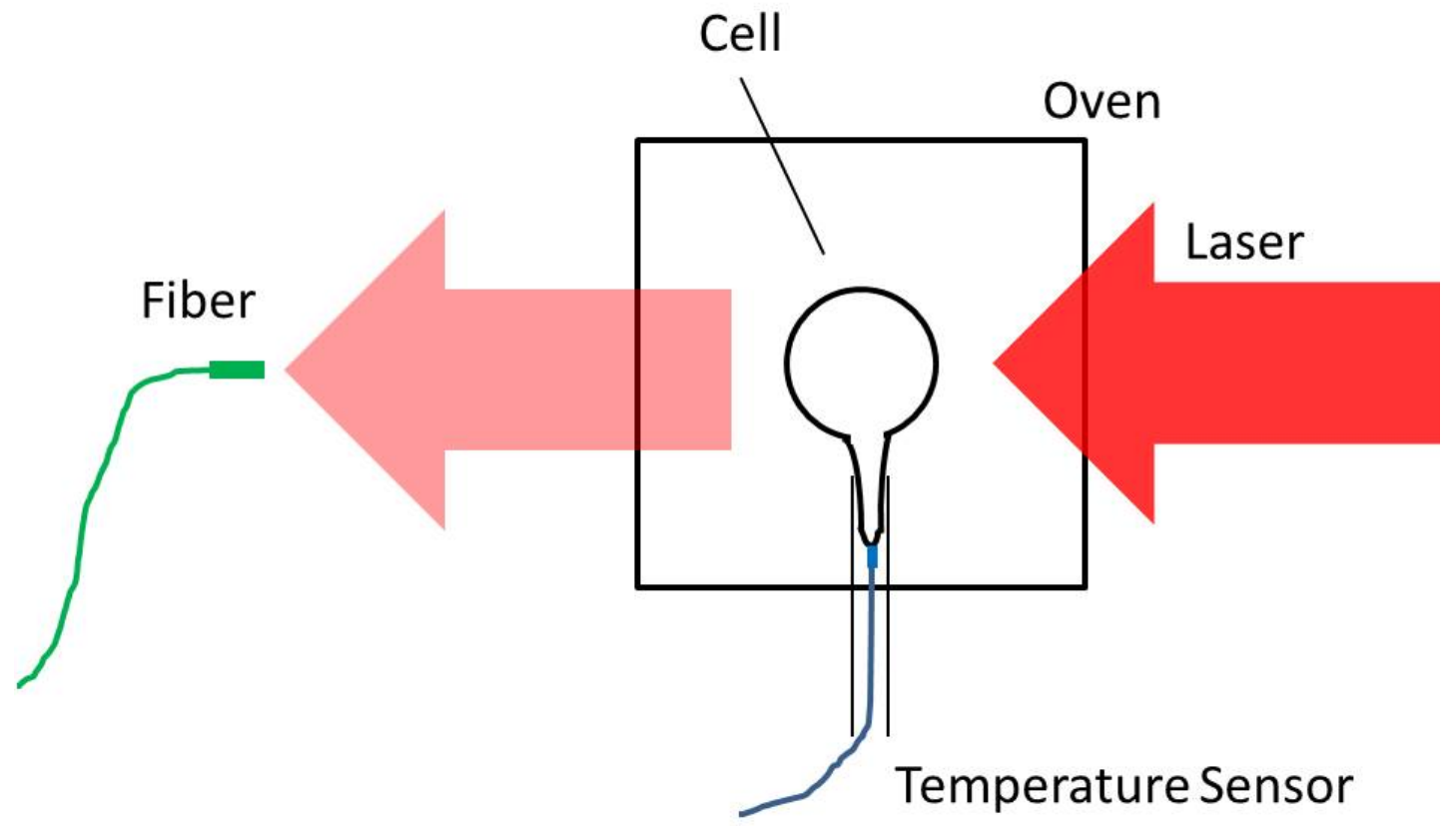

Figure 3.6: Measuring the laser absorption spectrum at the back of the heated oven.

As shown in Fig. 3.6, the cell was illuminated by circularly polarized laser light while the oven was heated to $\sim 100^{\circ} \mathrm{C}$. A temperature sensor attached to the cell pulloff was used for temperature feedback. Note that the temperature inside the cell can be higher than the temperature measured at the cell pull-off due to laser heating. A fibre was placed at the back of the cell to sample the light coming through. The fibre was connected to a spectrometer to analyze the spectrum. The lasers were adjusted one by one to make sure their spectra were centered at the Rb absorption frequency, as shown in Fig. 3.7 


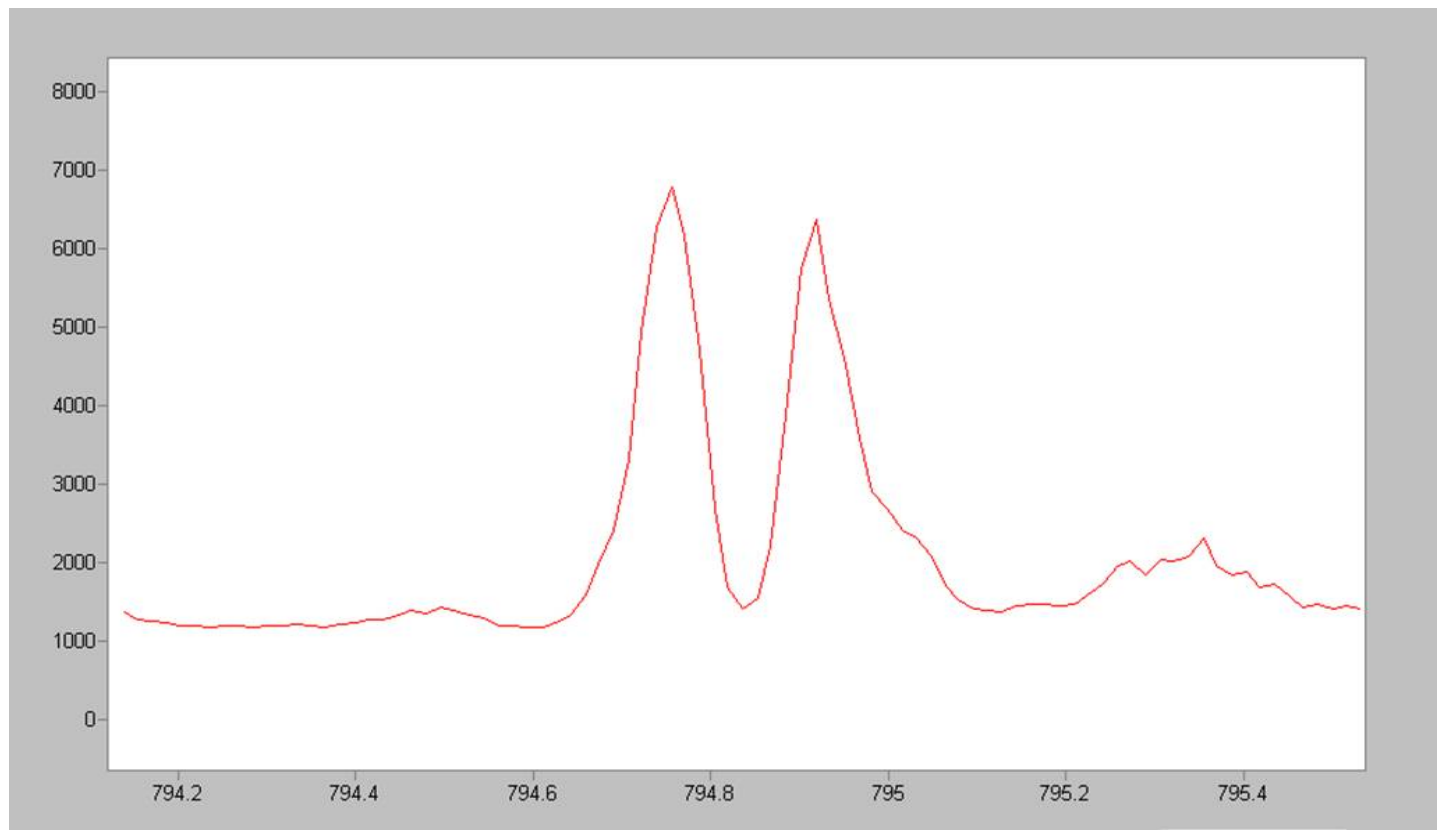

Figure 3.7: Laser spectrum measured behind cell $\mathrm{X} 4$. The $\mathrm{x}$ axis unit is $\mathrm{nm}$, the $\mathrm{y}$ axis is in some arbitrary unit. Note there is a $\sim 0.4 \mathrm{~nm}$ shift on the $\mathrm{x}$ axis.

\subsection{3 $\quad{ }^{129}$ Xe Polarimetry}

We have already discussed the physics of EPR polarimetry earlier. In this section, I will talk about technical details and present the results of our Xe polarization study.

\subsubsection{EPR Technical Details}

The most straightforward way to perform Xe polarimetry in our case would have been to measure the EPR frequency shift of a Xe cell. However, the frequency shift enhancement factor of $\mathrm{Rb}^{129} \mathrm{Xe}$ is not known very accurately. The value reported in [59] has $\mathrm{a} \sim 6.3 \%$ error, and the temperature and pressure dependence has not yet been fully established. On the other hand, $\kappa_{0}$ for $\mathrm{Rb}^{3}{ }^{3} \mathrm{He}$ has been measured accurately [55], the error is about $1.5 \%$ and the temperature dependence is well known over the range relevant to our experiment. We were therefore able to make a more accurate measurement of the Xe polarization by first measuring the polarization calibration constant (which equals polarization divided by the corresponding NMR signal size) of a ${ }^{3} \mathrm{He}$ cell, and then convert it to calibration constants for Xe cells based on the different gyromagnetic ratios, cell geometries, gas densities and system gains. These Xe cell calibration 
constants can in turn be used to calculate Xe polarizations from Xe NMR signals.

Our setup for performing EPR polarimetry and pulsed NMR are shown in Fig. 3.8 . We will focus on EPR measurements in this section and talk about pulsed NMR mea-



Figure 3.8: Our Setup for EPR polarimetry and Pulsed NMR. The EPR electronics are shown on the left and the PNMR electronics are shown on the right.

surements in Section 3.3 .3 .2

The first step in our EPR measurements was to locate the desired Zeeman transition. From Eq. 3.22 , we roughly knew what the transition frequency was, and we performed a so-called "FM sweep" in a small frequency range nearby. For example we swept the frequency from $3.55 \mathrm{MHz}$ to $3.70 \mathrm{MHz}$ to look for the ${ }^{85} \mathrm{Rb}\left(\mathrm{F}=3, \mathrm{~m}_{F}=3\right) \rightarrow(\mathrm{F}=3$, $\mathrm{m}_{F}=2$ ) transition in a $\sim 8$ Gauss holding field. Basically, RF frequency generated by the VCO was broadcasted to the cell, and we analyzed the $\mathrm{D}_{2}$ fluorescence as the VCO frequency changed. For FM sweeps, we did not send the lock-in amplifier output to the Proportional-Integral feedback box (P. I. Box), so the VCO input signal was a purely 
AC signal. The VCO output signal was:

$$
f_{v c o}(t)=f_{0}(t)+\Delta f \cos \left(f_{m} t\right)
$$

where $f_{0}$ was the VCO central frequency, $\Delta f$ was the modulation amplitude and $f_{m}$ was the modulation frequency, which we usually used $200 \mathrm{~Hz}$. The intensity of D2 fluorescence was proportional to the depolarization rate induced by the RF frequency.

Assuming a perfect Lorentzian line shape for the EPR transition, the D2 light intensity can be described by:

$$
I(f) \propto \frac{1}{\left(f-f_{0}\right)^{2}+\Gamma^{2}},
$$

where $\mathrm{f}$ is the RF frequency, $\mathrm{f}_{0}$ is the central frequency of the transition, and $\Gamma$ is the line width. Plugging Eq. 3.28 into Eq. 3.29, we have the D2 intensity during the FM sweep. Under the small modulation assumption, we can Taylor expand the D2 intensity with respect to the VCO central frequency:

$$
I\left(f_{v c o}(t)\right)=I\left(f_{0}(t)\right)+\left.\frac{\partial I}{\partial f}\right|_{f_{0}(t)} \Delta f \cos \left(f_{m} t\right)+\cdots .
$$

We used a lock-in amplifier to pick out the oscillating component at frequency $\mathrm{f}_{m}$, and the resulting signal was:

$$
\left.s(t) \propto \frac{\partial I}{\partial f}\right|_{f_{0}(t)} \cdot
$$

A plot of a Lorentzian function and its derivative is shown in Fig. 3.9. In the case of a FM sweep, the Lorentzian function can be interpreted as the D2 light intensity, and its derivative can be interpreted as the lock-in amplifier output signal (FM sweep amplitude). As shown in Fig. 3.9, the FM sweep signal amplitude goes across zero at the maximum D2 light intensity. We usually adjusted the phase of lock-in amplifier so that the FM sweep signal was inverted and the slope at resonance was positive. Fig. 3.10 shows a real FM sweep taken in our lab.

Now we consider how to lock to the resonance frequency as the effective holding field changed. First we set the VCO central frequency to the resonance frequency located by a FM sweep. Then we sent the output of the lock-in amplifier to the P. I. Box (Fig. 3.11). The VCO output would have another component that was a measure of the degree of off resonance. If the effective holding field changed, this off-resonance component would be non-zero and the VCO frequency would be adjusted to match the true resonance. 


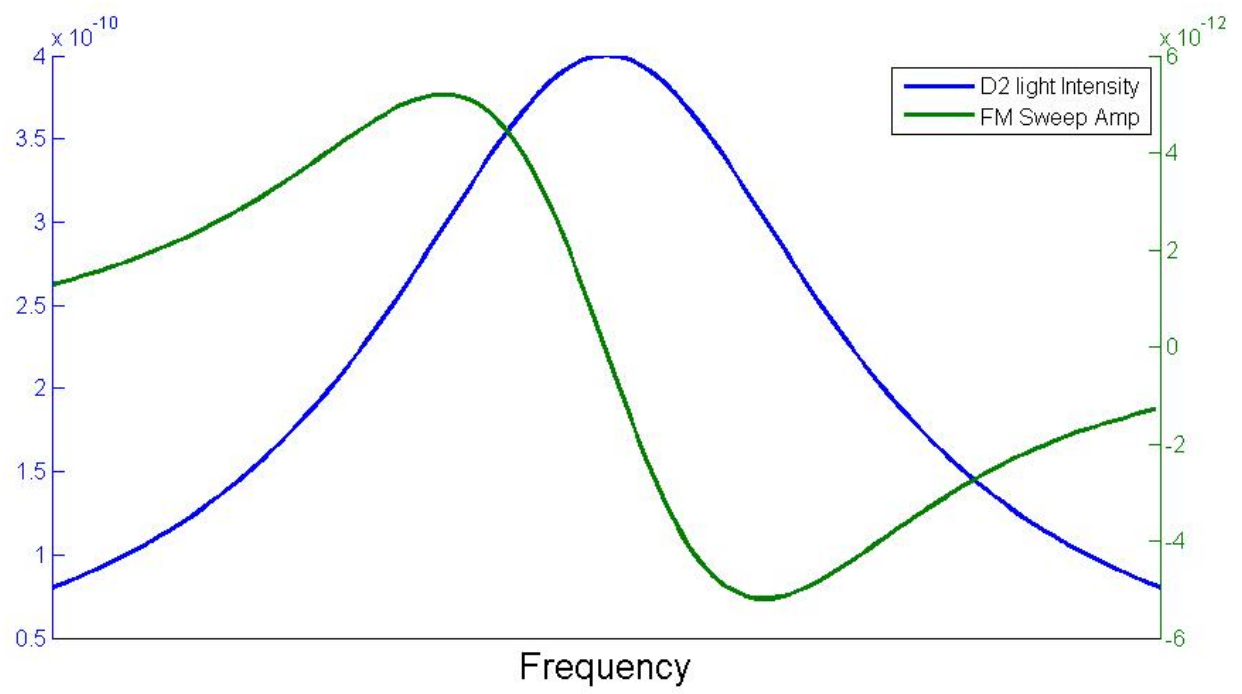

Figure 3.9: A Lorentzian function and its derivative.

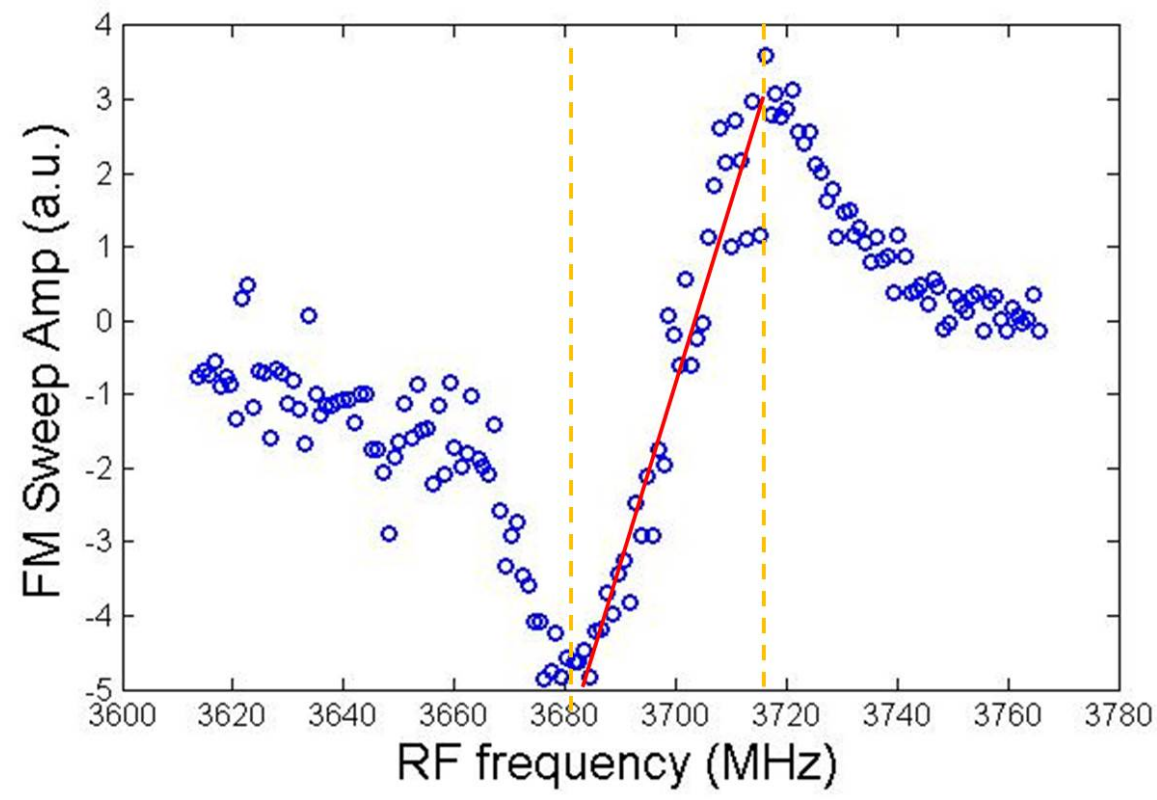

Figure 3.10: An real FM sweep taken in our lab. The cell diameter was 1", and the ${ }^{3} \mathrm{He}$ density was 2.56 amagats. It was illuminated by $\sim 20 \mathrm{~W}$ of spectrally narrowed laser power, and the oven temperature was set at $150^{\circ} \mathrm{C}$. The central linear region was fitted to a line. The EPR transition frequency is where it passes zero. 


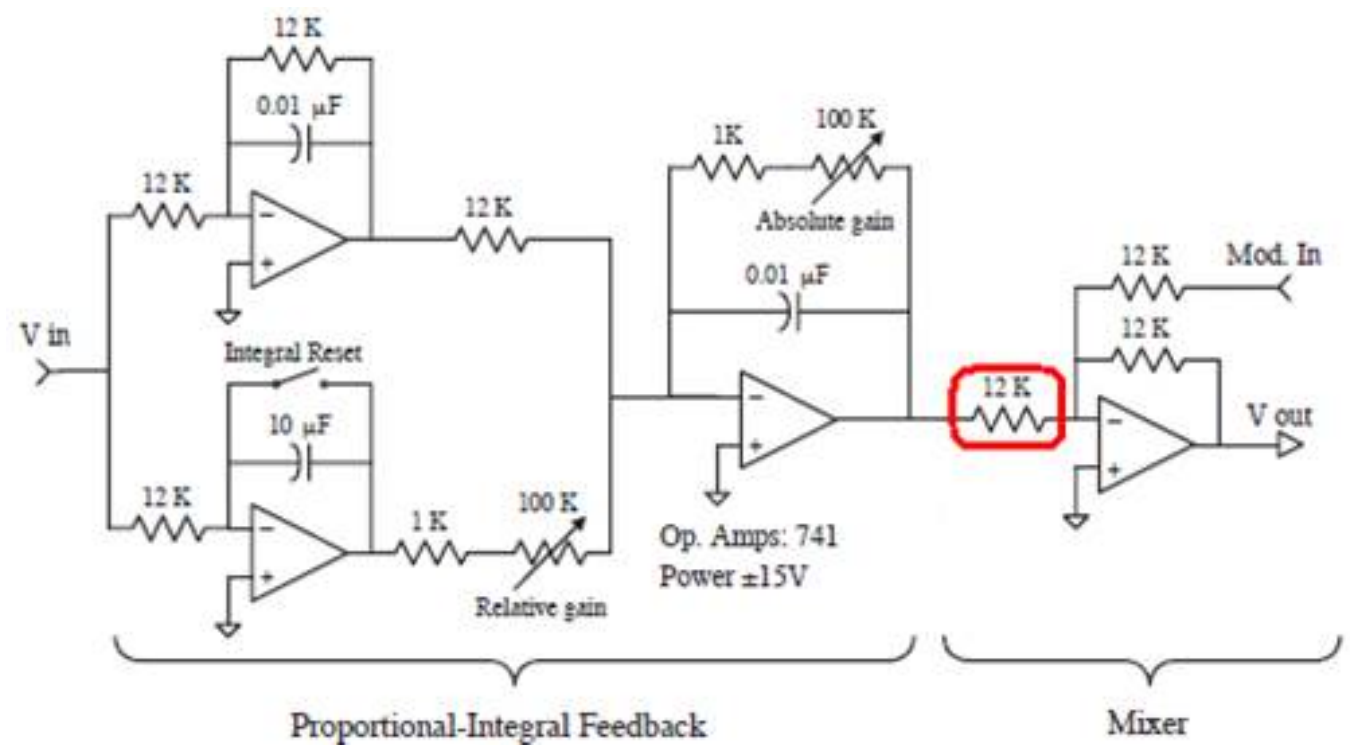

Figure 3.11: The Proportional-Integral Feedback circuit. The PI circuit used in our lab is the same one used by Romalis [60. Previous students in our lab noted that Romalis probably omitted a $12 \mathrm{~K}$ resistor when describing the this circuit. The diagram here is adapted from Dolph's thesis [19].

In EPR polarimetry, the frequency shifts were caused by the change of the magnetization of noble gas nuclei. For ${ }^{3} \mathrm{He}$, we first let the polarization build up close to saturation by SEOP, which usually took 5-10 hrs. We then locked the VCO frequency to the EPR frequency and flipped the spins by 180 degrees using AFP. We performed an RF frequency sweep rather than a holding field sweep here to keep the external magnetic field constant. The $\mathrm{Rb}$ atoms felt a different total magnetic field after the ${ }^{3}$ He spins were flipped, and the EPR transition frequency changed accordingly. The EPR frequency was tracked by the VCO frequency, which was measured by a frequency counter and recorded by a computer (Fig. 3.12). The ${ }^{3}$ He polarization was then calculated by Eq. 3.27. Higher order corrections were also included for better accuracy. Note an extra factor of 0.5 should be included since the ${ }^{3} \mathrm{He}$ magnetization was flipped by $180^{\circ}$.

Xe polarization builds up much more quickly due to faster spin exchange and other relaxation mechanisms. Fig. 3.13 shows a Xe polarization evolution curve measured under our typical SEOP conditions (the "spin-up" curve). With a spin-up time constant as short as $20 \mathrm{~s}$, we locked the VCO frequency to the EPR transition with completely 


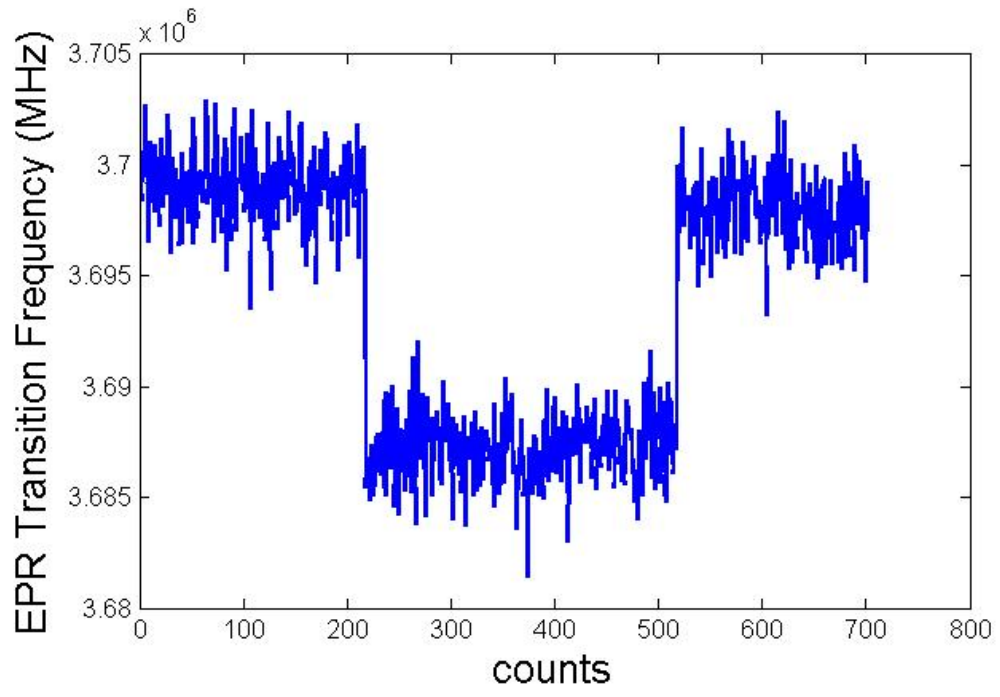

Figure 3.12: An EPR frequency shift measurement. This cell had 2.56 amagats of ${ }^{3} \mathrm{He}$. The oven temperature was at $150^{\circ} \mathrm{C}$. The frequency shifted when the ${ }^{3} \mathrm{He}$ magnetization was flipped.

unpolarized Xe, and monitored how the VCO frequency changed as the Xe nuclei become polarized. The whole process only took several minutes. The VCO frequency at the beginning (with unpolarized Xe) and the end (with maximally polarized Xe) were used to calculate Xe polarization.

\subsubsection{Pulsed NMR Technical Details}

In this section we'll talk about the pulsed NMR setup in our lab. The electronics for pulsed NMR is shown on the right side of Fig. 3.8. A gate signal was generated by a National Instrument Data Acquisition (DAQ) card equipped on a PC, with both input and output capabilities. A function generator was gated by the this signal and sent out an RF pulse with pre-set amplitude and frequency when the gate voltage was high. The RF pulse was amplified by an RF amplifier and then sent to the RF coil. The noble gas nuclei was flipped by some angle, depending on the amplitude and duration of the $\mathrm{RF}$ pulse. In general there was a transverse magnetization component, which precessed along the holding field and induced a voltage in the detection coil. Unlike some pulsed NMR studies that used the same coil for both broadcasting RF and detecting the spin 


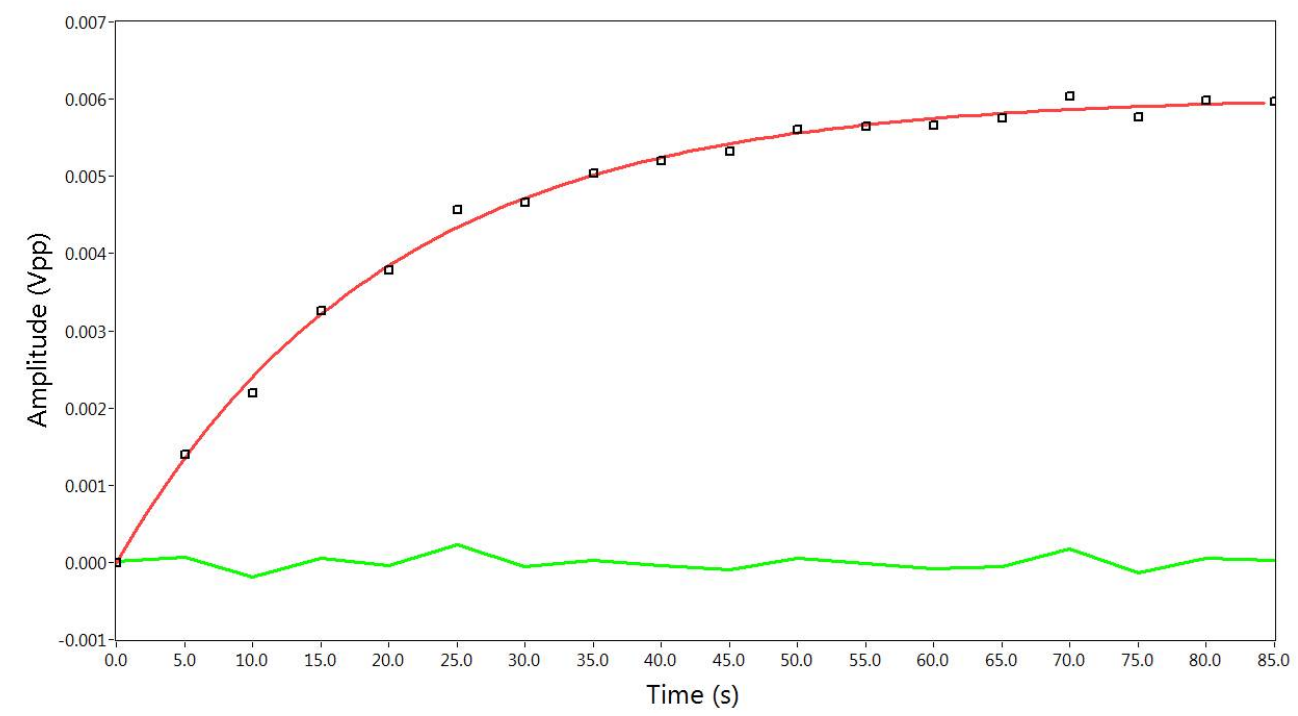

Figure 3.13: A spinup curve taken with cell $\mathrm{X} 2$ at $70^{\circ} \mathrm{C}$ with 3 spectrally narrowed lasers. This curve is fitted to a model $A\left(1-e^{-t / B}\right)+C$. The red line is the fitted function and the green line is the residual. The time constant is found to be about $20 \mathrm{~s}$.

precession signal [47], we used two separate coils for transmit and receive. The signal induced in the detection coil was first sent through a low noise pre-amplifier, and then to an isolation switch. The isolation switch was also controlled by the gate signal, and only allowed a signal to pass through when the gate voltage was low, which helped to avoid saturating downstream electronics while RF was broadcasted. The signal, which oscillated at the Larmor frequency $\left(\mathrm{f}_{1}\right)$, was then sent to a frequency mixer. Another input of the mixer was connected to a function generator that generated a frequency $\left(f_{2}\right)$ slightly different from the Larmor frequency. The output of the mixer had two frequency components $\left(f_{1}+f_{2}\right)$ and $\left(f_{1}-f_{2}\right)$. After filtering out the high frequency component and further amplifying the low frequency component by a second pre-amplifier, the signal was digitized by an input channel of the DAQ card and recorded on the PC. Fig. 3.14 shows a typical FID signal from a pulsed NMR measurement. The first $10 \mathrm{~ms}$ of data was left out from the analysis since there was an obvious signal corruption due to the detection coils ringing down and electronics saturation. We fitted the rest data to an oscillating exponential decay model $S \cos (f t+\phi) \exp \left(-\frac{t}{\tau}\right)$, where $S$ was the signal size at the beginning of the fit, $f$ was the oscillation frequency, $\phi$ was an initial phase, and 
$\tau$ was the decay time constant.



Figure 3.14: Free Induction Decay signal taken with cell $\mathrm{X} 4$ at $68^{\circ} \mathrm{C}$ with 3 spectrally narrowed lasers. The temperature was measured at the cell pull-off.

It is also important to know the tip angle at which a FID signal is measured. Although in principle tip angles can be calculated as long as we know the RF pulse width and strength (Eq. 3.15), it can also be measured conveniently. Basically, we took a series of FID measurements with the same RF pulse in a period of time short enough that the spin relaxation effects can be ignored. The signal size decay between successive measurements was thus mostly due to the loss of FID measurements. For a tip angle $\theta$, only a fraction of $\cos \theta$ of the polarization survived after each measurement. The transverse magnetization was proportional to the product of $\sin \theta$ and the current polarization. Therefore the amplitude of the $\mathrm{n}_{t h}$ FID was:

$$
S(n)=S_{0} \cos ^{n-1} \theta \sin \theta
$$

where $S_{0}$ was the signal size corresponding to the maximum transverse magnetization (i.e., if $90^{\circ}$ tip angle was applied for the first measurement). We can thus determine the tip angle and $\mathrm{S}_{0}$ by fitting the FID amplitudes to Eq. 3.32 , as shown in Fig. 3.15 . 
We can now calculated the "Total Initial Amplitude (TIA)":

$$
S_{T I A}=\frac{S(1)}{\sin \theta e^{-\frac{t_{c u t}}{\tau}}}=\frac{S_{0}}{e^{-\frac{t_{c u t}}{\tau}}},
$$

where $\tau$ was the decay time constant determined by fitting the FID signals to a damped oscillation model, and $t_{c u t}$ was the time omitted at the beginning (Fig. 3.14). $\mathrm{S}_{\text {TIA }}$ corresponded to the FID signal size immediately after the spins were flipped to the transverse plane and was thus independent of $\mathrm{T}_{2}^{*}$ and $\theta$.

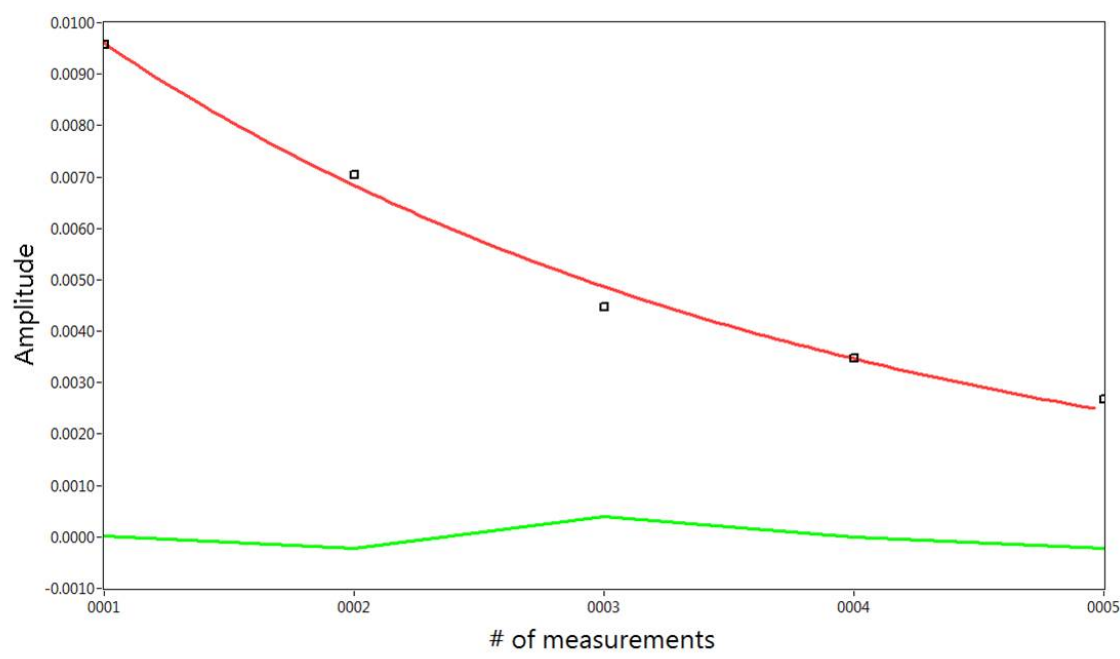

Figure 3.15: Tipangle measurements taken with cell $\mathrm{X} 4$ at $68^{\circ} \mathrm{C}$ with 3 spectrally-narrowed lasers. Five successive FID measurements were included in this fit. The red line is the fitted function $S(n)=S_{0} \cos ^{n-1} \theta \sin \theta$, and the green line is the residual. From the fitting result, we found $\theta=43.3^{\circ}$.

\subsubsection{Calibration Constants}

A polarization Calibration Constant (C.C.) is a quantity that relates nuclear polarizations to NMR signal sizes by $P=C . C . \cdot S$. As we discussed earlier, the enhancement factor $\kappa_{0}$ for $\mathrm{Rb}-{ }^{3} \mathrm{He}$ is much better understood than that of $\mathrm{Rb}^{129}{ }^{129} \mathrm{Xe}$ pairs, therefore it would be more accurate to measure the C.C. of a ${ }^{3} \mathrm{He}$ cell first, and then derive from it the C.C. for Xe cells by considering factors contributing to NMR signal sizes. The C.C. of Xe cells can then be used to determine Xe polarizations from NMR signals.

Assuming nuclear polarization $\mathrm{P}_{\mathrm{He}}$ is measured in a cell with a ${ }^{3} \mathrm{He}$ density of $\rho_{\mathrm{He}}$ and a volume of $V_{\mathrm{He}}$. The total magnetization is thus $M_{\mathrm{He}}=\left(\gamma_{\mathrm{He}} J_{\mathrm{He}}\right) \rho_{\mathrm{He}} V_{\mathrm{He}} P_{\mathrm{He}}$, 
where $\gamma_{\mathrm{He}} J_{\mathrm{He}}$ is the magnetic moment of a ${ }^{3} \mathrm{He}$ nucleus. The FID signal size from such a cell can be expressed:

$$
S_{\mathrm{He}}=g 1_{\mathrm{He}} g 2_{\mathrm{He}} f_{\mathrm{He}} M_{\mathrm{He}}=g 1_{\mathrm{He}} g 2_{\mathrm{He}}\left(\gamma_{\mathrm{He}} B_{\mathrm{He}}\right)\left(\gamma_{\mathrm{He}} J_{\mathrm{He}}\right) \rho_{\mathrm{He}} V_{\mathrm{He}} P_{\mathrm{He}}
$$

where $\mathrm{S}_{\mathrm{He}}$ is the total initial amplitude (TIA, which is defined in Sec. 3.3.3.2). In this section, I will simply refer to the total initial amplitude by "FID size". Here $g 1_{\mathrm{He}}$ is the gain from the cell and detection coil geometry, and $g 2_{H e}$ is the gain due to the detection coil circuit and electronics. Also $f_{\mathrm{He}}$ is the Larmor frequency, which is the product of the gyromagnetic ratio $\gamma_{\mathrm{He}}$ and the holding field $\mathrm{B}_{\mathrm{He}}$.

Similarly, the FID signal size of a ${ }^{129} \mathrm{Xe}$ cell is:

$$
S_{\mathrm{Xe}}=g 1_{\mathrm{Xe}} g 2_{\mathrm{Xe}}\left(\gamma_{\mathrm{Xe}} B_{\mathrm{Xe}}\right)\left(\gamma_{\mathrm{Xe}} J_{\mathrm{Xe}}\right) \rho_{\mathrm{Xe}} V_{\mathrm{Xe}} P_{\mathrm{Xe}}
$$

where we emphasize that $\rho_{\mathrm{Xe}}$ is the density of ${ }^{129} \mathrm{Xe}$ only, and other Xe isotopes are not included.

By comparing ${ }^{129} \mathrm{Xe}$ and ${ }^{3} \mathrm{He}$ FID signal sizes, Xe polarization can be calculated:

$$
\begin{aligned}
P_{\mathrm{Xe}} & =\frac{P_{\mathrm{He}}}{S_{\mathrm{He}}} \frac{\rho_{\mathrm{He}}}{\rho_{\mathrm{Xe}}} \frac{V_{\mathrm{He}}}{V_{\mathrm{Xe}}} \frac{\gamma_{\mathrm{He}}}{\gamma_{\mathrm{Xe}}}\left(\frac{\gamma_{\mathrm{He}} B_{\mathrm{He}}}{\gamma_{\mathrm{Xe}} B_{\mathrm{Xe}}}\right) \frac{g 1_{\mathrm{He}}}{g 1_{\mathrm{Xe}}} \frac{g 2_{\mathrm{He}}}{g 2_{\mathrm{Xe}}} S_{\mathrm{Xe}} \\
& =C_{\mathrm{Xe}} S_{\mathrm{Xe}}
\end{aligned}
$$

where $\mathrm{C}_{\mathrm{Xe}}$ is the calibration constant for the $\mathrm{Xe}$ cell of interest. $\mathrm{C}_{\mathrm{Xe}}$ can be separated into two parts:

$$
\begin{aligned}
C_{\mathrm{Xe}} & =C_{\mathrm{He}} C_{m i s c}, \\
\text { where } C_{\mathrm{He}} & =\frac{P_{\mathrm{He}}}{S_{\mathrm{He}}} \\
\text { and } C_{\text {misc }} & =\frac{\rho_{\mathrm{He}}}{\rho_{\mathrm{Xe}}} \frac{V_{\mathrm{He}}}{V_{\mathrm{Xe}}} \frac{\gamma_{\mathrm{He}}}{\gamma_{\mathrm{Xe}}}\left(\frac{\gamma_{\mathrm{He}} B_{\mathrm{He}}}{\gamma_{\mathrm{Xe}} B_{\mathrm{Xe}}}\right) \frac{g 1_{\mathrm{He}}}{g 1_{\mathrm{Xe}}} \frac{g 2_{\mathrm{He}}}{g 2_{\mathrm{Xe}}} .
\end{aligned}
$$

$\mathrm{C}_{\mathrm{He}}$ is the C.C. for the ${ }^{3} \mathrm{He}$ cell, and $\mathrm{C}_{\text {misc }}$ accounts for all other factors, which are different for each Xe cell.

$\mathrm{C}_{\mathrm{He}}$ can be determined by performing EPR polarimetry and pulsed NMR at the same ${ }^{3} \mathrm{He}$ polarization. Our basic procedure for measuring $\mathrm{C}_{\mathrm{He}}$ is described below:

1. Install a ${ }^{3} \mathrm{He}$ cell in the oven.

2. Polarize the ${ }^{3} \mathrm{He}$ to the saturation polarization by SEOP. 
3. Measure FID signal size.

4. Measure the ${ }^{3} \mathrm{He}$ polarization by EPR polarimetry.

5. Measure FID signal size again.

From EPR polarimetry we measured two polarizations corresponding to flipping the spins back and forth. As shown in Fig. 3.12, the two frequency shifts were a little bit different due to some polarization loss during an EPR measurement. Therefore we measured FID signals before and after EPR polarimetry. For the FID measurements taken before EPR, we were interested in the signal size related to the ${ }^{3} \mathrm{He}$ polarization immediately before EPR polarimetry. However, this signal amplitude cannot be measured directly since some polarization would be lost due to any FID measurement. Instead, we used the tip angle extracted from a series of FID measurements and extrapolated the amplitude for a "future" FID, which was not actually measured. The FID signal size after EPR polarimetry can be measured directly. We then used the average frequency shift and the average FID amplitude to calculate the ${ }^{3} \mathrm{He}$ calibration constant $C_{\mathrm{He}}=\frac{P_{\mathrm{He}}}{S_{\mathrm{He}}}$.

Now let's consider $\mathrm{C}_{m i s c}$. We know the noble gas densities (for Xe we need to use the density of isotope ${ }^{129} \mathrm{Xe}$ ) and cell volumes when filling the cells, and we also know the gyromagnetic ratios. $\gamma_{\mathrm{He}} B_{\mathrm{He}}$ and $\gamma_{\mathrm{Xe}} B_{\mathrm{Xe}}$ are the respective Larmor frequencies of ${ }^{3} \mathrm{He}$ and ${ }^{129} \mathrm{Xe}$. We chose different holding fields so that the Larmor frequencies were the same for ${ }^{3} \mathrm{He}$ and ${ }^{129} \mathrm{Xe}$. The main advantage of using the same Larmor frequency is that the circuit and electronics gains are the same. Thus we have $\frac{\gamma_{\mathrm{He}} B_{\mathrm{He}}}{\gamma_{\mathrm{Xe}} B_{\mathrm{Xe}}}=1$ and $\frac{g 2_{\mathrm{He}}}{g 2_{\mathrm{Xe}}}=1 . \mathrm{g} 1_{\mathrm{He}}$ and $\mathrm{g} 1_{\mathrm{Xe}}$ are the gains due to cell and detection coil geometry. These gains can be quite complicated in general. However, in our case we used the same detection coil for both noble gases. Moreover, for a spherical cell, the magnetic field outside the sphere is the same as that caused by a point-like dipole moment equal to the total dipole moment of the cell [57]. Therefore sizes of the spherical cells don't matter either and we have $\frac{g 1_{\mathrm{He}}}{g 1_{\mathrm{Xe}}}=1$. By combining $\mathrm{C}_{m i s c}$ with $\mathrm{C}_{\mathrm{He}}$, we can determine the calibration constants for cells X1-4 listed Tab. 3.2.

Alternatively, we can perform EPR polarimetry on a Xe cell, and then relate its calibration constant $\mathrm{C}_{\mathrm{XeCal}}$ to the C.C. of other Xe cells. Due to the fast SEOP rate, the ${ }^{129} \mathrm{Xe}$ spins were not flipped during polarimetry. Instead, EPR frequencies with zero and maximum Xe polarization were measured, and their difference was the frequency 
shift due to saturated Xe magnetization. We then measured the FID amplitude at the saturated $\mathrm{Xe}$ polarization to determine $\mathrm{C}_{\mathrm{XeCal}}$, which was in turn used to calculate $\mathrm{C}_{\mathrm{Xe}}$ for other Xe cells using equations similar to Eq. (3.38), 3.39) and (3.40).

C.C. for cells X1-4 are shown in Tab 3.3 together with their errors at the end of Sec. 3.3.3.4.

\subsubsection{Errors of the Calibration Constants}

The error of the final calibration constant $\mathrm{C}_{\mathrm{Xe}}$ needs to be analyzed carefully. I will take $\mathrm{C}_{\mathrm{Xe}}$ derived from ${ }^{3} \mathrm{He}$ polarimetry for example and discuss error analysis. Let's consider the errors in $\mathrm{C}_{\mathrm{He}}$ and $\mathrm{C}_{\text {misc }}$ separately.

$$
C_{H e}=\frac{P_{\mathrm{He}}}{S_{\mathrm{He}}}=C_{1} \frac{\Delta \nu}{\kappa_{0} \rho_{\mathrm{He}}} \frac{1}{S_{\mathrm{He}}}
$$

- $\mathrm{C}_{1}$ : A coefficient with negligible error.

- $\frac{1}{\kappa_{0}}: 1.5 \%$ systematic error, as reported in [21].

- $\Delta \nu: \sim 3 \%$ statistical error. An EPR measurement typically has the shape of a hat or a well, as shown in Fig. 3.12, We took the average of 100 points before tipping the spins $\left(f_{1}\right)$, after flipping the spins $\left(f_{2}\right)$ and after finally flipping them back $\left(f_{3}\right)$ as the three EPR transition frequencies. The average frequency shift was estimated as $\frac{f_{1}+f_{3}}{2}-f_{2}$. The errors of $f_{1}$ and $f_{3}$ were first combined for a reduced error, then the error of $\mathrm{f}_{2}$ was added in quadrature.

- $\frac{1}{S_{\mathrm{He}}}: \sim 1.1 \%$ statistical error. We measured $\mathrm{S}_{\mathrm{He}}$ before and after EPR polarimetry. The errors of the NMR signal sizes came from the fit of FID signals (Fig. 3.14) and tip angles (Fig. 3.15). We took the average of the two values and used the reduced error.

- $\frac{1}{\rho_{\mathrm{He}}}: 2 \%$ systematic error. It cancels with a factor in $\mathrm{C}_{m i s c}$ and is ignored here.

We have four sets of separate $\mathrm{C}_{\mathrm{He}}$ measurements, and the four calibration constants are $0.445(9) \% / \mathrm{mV}, 0.482(18) \% / \mathrm{mV}, 0.420(14) \% / \mathrm{mV}$ and $0.454(19) \% / \mathrm{mV}$. The errors quoted here only include statistical errors, and they cannot cover the spread of the results, which suggests there are other errors that we haven't considered. Therefore we took the average of the four calibration constants and used their standard deviation 
as the final statistical error. The small systematic error of $\kappa_{0}$ was then combined in quadrature. The final C.C. for the ${ }^{3} \mathrm{He}$ cell is $\mathrm{C}_{\mathrm{He}}=0.450(26) \% / \mathrm{mV}$.

Now let's consider $\mathrm{C}_{m i s c}$ :

$$
C_{m i s c}=\frac{\rho_{\mathrm{He}}}{\rho_{\mathrm{Xe}}} \frac{V_{\mathrm{He}}}{V_{\mathrm{Xe}}} \frac{\gamma_{\mathrm{He}}}{\gamma_{\mathrm{Xe}}}\left(\frac{\gamma_{\mathrm{He}} B_{\mathrm{He}}}{\gamma_{\mathrm{Xe}} B_{\mathrm{Xe}}}\right) \frac{g 1_{\mathrm{He}}}{g 1_{\mathrm{Xe}}} \frac{g 2_{\mathrm{He}}}{g 2_{\mathrm{Xe}}}
$$

The errors associated with $\mathrm{C}_{m i s c}$ are:

- $\rho_{\mathrm{He}}: 2 \%$ error, it cancels with the $\frac{1}{\rho_{\mathrm{He}}}$ factor in $\mathrm{C}_{\mathrm{He}}$ and is therefore ignored.

- $\rho_{\mathrm{Xe}}: 2 \%$ error.

- $\frac{V_{\mathrm{He}}}{V_{\mathrm{Xe}}}: 3 \%$ error.

- $\frac{\gamma_{\mathrm{He}}}{\gamma_{\mathrm{Xe}}}$ : negligible error.

- $\frac{\gamma_{\mathrm{He}} B_{\mathrm{He}}}{\gamma_{\mathrm{Xe}} B_{\mathrm{Xe}}}$ : small $(\sim 0.1 \%)$ error in Larmor frequencies.

- $\frac{g 1_{\mathrm{He}}}{g 1_{\mathrm{Xe}}}: 2 \%$ error. This error takes into account the fact that the cells are not perfect spheres.

- $\frac{g 2 \mathrm{He}}{g 2 \mathrm{xe}}: 1.5 \%$ error due to the frequency dependence of the gain of the receive chain. Combining the above errors in quadrature, $\mathrm{C}_{\text {misc }}$ has a relative error of $4.4 \%$.

The C.C. for a Xe cell is the product of $\mathrm{C}_{\mathrm{He}}$ and $\mathrm{C}_{m i s c}$, and its error is calculated by adding the relative error of $\mathrm{C}_{\mathrm{He}}$ and $\mathrm{C}_{m i s c}$ in quadrature.

The error of $\mathrm{C}_{\mathrm{Xe}}$ derived from ${ }^{129} \mathrm{Xe}$ EPR polarimetry can be analyzed similarly. The C.C. of Xe cells X1-4 measured by the two different methods are summarized in Table. 3.3

\begin{tabular}{c|cc|cc}
\hline \hline & \multicolumn{2}{|c|}{${ }^{3} \mathrm{He}$ EPR } & \multicolumn{2}{c}{${ }^{129} \mathrm{Xe}$ EPR } \\
Cell & C.C.HeEPR $(\% / \mathrm{mV})$ & Err $(\% / \mathrm{mV})$ & C.C.XeEPR $(\% / \mathrm{mV})$ & Err $(\% / \mathrm{mV})$ \\
\hline $\mathrm{X} 1$ & 5.69 & 0.41 & 6.70 & 0.55 \\
$\mathrm{X} 2$ & 3.14 & 0.23 & 3.70 & 0.31 \\
$\mathrm{X} 3$ & 2.11 & 0.15 & 2.49 & 0.21 \\
$\mathrm{X} 4$ & 1.59 & 0.12 & 1.87 & 0.15 \\
\hline \hline
\end{tabular}

Table 3.3: Polarization calibration constants and their errors for Xe cells X1-4 derived from either ${ }^{3} \mathrm{He}$ or ${ }^{129} \mathrm{Xe}$ EPR polarimetry. 
As shown in Tab. 3.3, the calibration constants measured using the two different methods do not agree very well. The main reason is that $\left(\kappa_{0}\right)_{\mathrm{RbXe}}$ is still not well understood, and the $\left(\kappa_{0}\right)_{\mathrm{RbXe}}$ value we used was measured by Ma et.al. under different conditions [59]. For example, their Xe pressure was only about 10 Torr, and they studied the temperature range between $140^{\circ} \mathrm{C}$ and $220^{\circ} \mathrm{C}$. They also reported in the same paper an anomalous decrease of $\left(\kappa_{0}\right)_{\mathrm{RbXe}}$ at higher Xe densities ( 100 Torr $)$ and high temperatures $>160^{\circ} \mathrm{C}$ ), and there was no plausible theoretical explanation. On the other hand, $\left(\kappa_{0}\right)_{\mathrm{RbHe}}$ has been measured accurately by several groups [55, 58] with relative errors under $2 \%$ in temperature range relevant to our experiment. Therefore C.C. derived from ${ }^{3} \mathrm{He}$ EPR polarimetry is more reliable.

\subsubsection{Results and Discussions}

We measured the FID signals for cell X1-4 over a temperature range of about $60^{\circ} \mathrm{C}$. The FID amplitudes for each cell were converted to polarizations using the corresponding C.C.HeEPR. The temperature dependence of the Xe polarizations for all four cells are plotted in Fig. 3.16 .

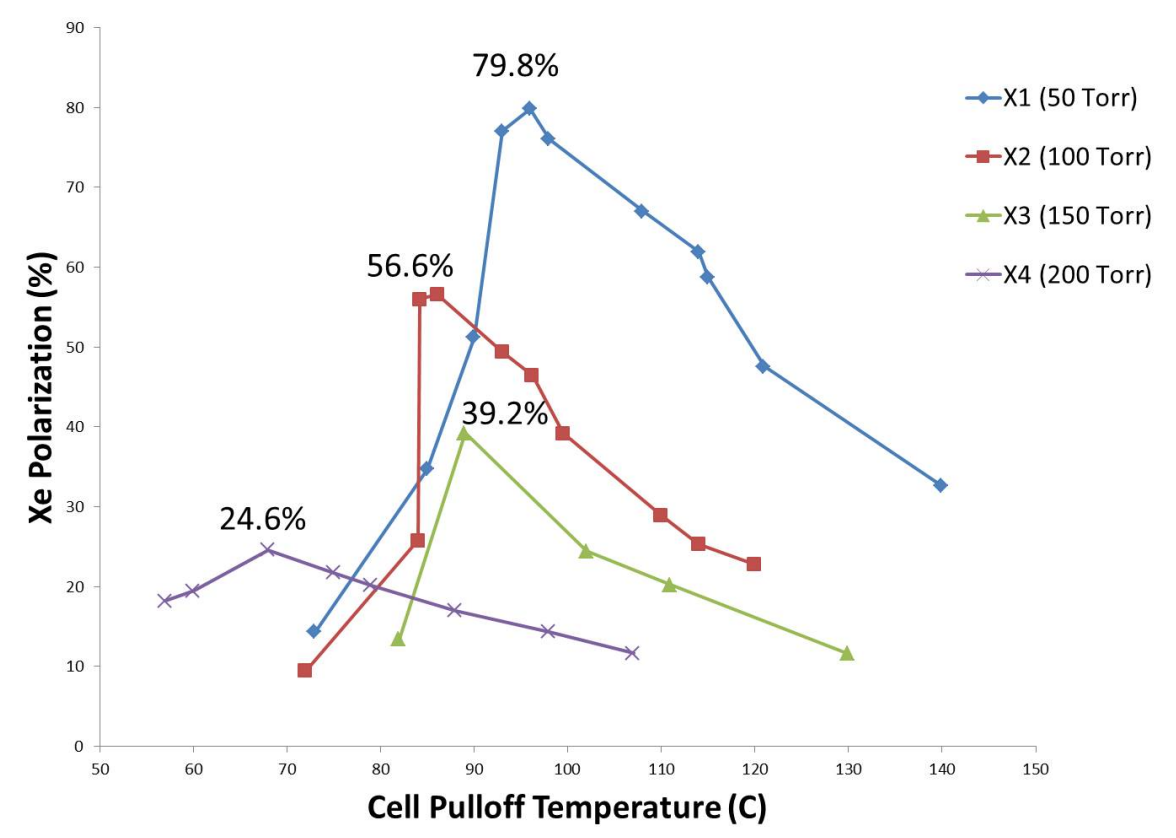

Figure 3.16: Xe polarization as a function of temperature for cells X1-4. We used $\sim 50 \mathrm{~W}$ of spectrally narrowed laser power for all the measurements. 
While one would assume the temperature dependence of polarization to be smoother (especially for cell X2), we note that the temperatures were measured at the cell pull-offs (Fig. 3.6), and they were presumably different from the temperature inside the cells which determined $\mathrm{Rb}$ density. The true cell temperature could have a complicated dependence on the oven temperature due to laser heating.

There are two interesting features in Fig. 3.16 .

1. The optimum temperature increases as the Xe pressure drops. Although in the graph cell X3 has a slightly higher optimum temperature than X2, we think it is related to how the temperature sensors were attached to the cell pull-offs and the fact that cell pull-off temperatures didn't always reflect the true cell temperature. As shown in Eq. 2.46, a high Xe polarization can be achieved only if the $\mathrm{Rb}$ polarization is high. However, $\mathrm{Xe}$ is very depolarizing to $\mathrm{Rb}$ (Sec. 2.2.6), and it becomes more difficult to maintain a high $\mathrm{Rb}$ polarization as the Xe pressure increases. Rb vapor with low polarization in turn absorbs more laser power (Eq.2.14). As the cell temperature increases, the Rb density increases and photon consumption becomes higher. Above a certain temperature there are not enough photons left to polarize the back of the cell. As a result, the overall $\mathrm{Rb}$ polarization begins to drop, and so is the Xe polarization.

2. The maximum Xe polarization is higher for cells with lower Xe pressure. There are several reasons for it. First, the Rb spin-destruction rate is smaller for cells with less Xe, which results in a higher Rb polarization. Second, since the optimum temperature increases as the Xe pressure drops, the Rb density at optimum temperature is higher for cells with less Xe. Therefore the Rb-Xe spin exchange rate becomes higher (Eq. 2.47 and 2.51). Third, the spin exchange coefficient due to van der Waals molecules also increases as the Xe (and $\mathrm{N}_{2}$ ) fraction in the gas mixture decreases (Eq. 2.51). For example, the vdW spin exchange constant in $\mathrm{X} 1$ is roughly twice of that in cell $\mathrm{X} 4$.

To sum up, we polarized a series of cells with different Xe pressures using spectrally narrowed lasers, and studied the ${ }^{129} \mathrm{Xe}$ polarization as a function of temperature for each cell. The optimum cell temperature and the maximum Xe polarization increases as the Xe pressure decreases. We measured a highest polarization of about $80 \%$ in a cell with 50 Torr of Xe at around $100^{\circ} \mathrm{C}$. This study provided some guidance on how much 
polarization to expect and how to achieve higher polarizations in the Xe cells used for the imaging studies in Ch. 4 and 5 . Our results can also provide useful references for future development of Xe polarizers. 


\section{4 \\ Low Field MRI of HP Gases}

We describe a homebuilt MRI system for imaging laser-polarized noble gases at a very low holding field of $2 \mathrm{mT}^{1}$. A unique feature of this system was the use of Maxwell coils ${ }^{2}$ oriented at so-called "magic angles" to generate the transverse magnetic field gradients, which provided a simple alternative to Golay coils? We imaged laser-polarized ${ }^{3} \mathrm{He}$ and ${ }^{129} \mathrm{Xe}$ phantom: ${ }^{4}$ using this system. ${ }^{3} \mathrm{He}$ phantom was imaged using a conventional gradient-echo pulse sequence, and the ${ }^{129} \mathrm{Xe}$ phantom was imaged with both a gradientecho and a fully phase-encoded pulse sequence. All data for the ${ }^{3} \mathrm{He}$ image was acquired with one SEOP cycle. On the other hand, xenon was repolarized many times during the imaging procedure due to its much smaller magnetization. By comparing the two Xe images collected with different sequences, we demonstrated that low-field HP gas image quality can be dramatically improved by combining a fully phase-encoded $k$-space acquisition with auxiliary measurements of system imperfections including $B_{0}$ field drift and gradient infidelity. Implications for in-vivo lung imaging using this system are also discussed.

\footnotetext{
${ }^{1}$ We will follow the MRI unit convention and use Tesla/Millitesla as the unit for magnetic field in this chapter.

${ }^{2}$ A Maxwell pair consists of two identical coils on the same axis but with opposite current directions.

${ }^{3}$ Golay coils are saddle shaped coils commonly used in MRI scanners to generate transverse gradients.

${ }^{4}$ Phantoms are specially designed objects that are imaged to evaluate, analyze, and tune the performance of various imaging devices.
} 


\subsection{Basics of MRI}

The signal of a Free Induction Decay (FID) can be expressed by Eq. 3.18.

$$
s(t)=C \omega_{0} \sin \alpha e^{i\left(\omega_{0} t+\phi\right)} e^{-t / T_{2}^{*}}
$$

where $\omega_{0}$ is the Larmor frequency, $\alpha$ is the flip angle, $\phi$ is the starting phase, $\mathrm{T}_{2}^{*}$ is the decay time constant and $\mathrm{C}$ is a constant accounting for the total magnetization, detection coil efficiency and the system gain.

Let's ignore the exponential decay for now. For 1D continuously distributed spin density, the total FID size is:

$$
s(t)=C^{\prime} \int s(x) e^{i 2 \pi f_{0} t} d x
$$

where we absorbed $\omega_{0} \sin \alpha e^{i \phi}$ into the new constant C' and converted the unit of the frequency from radian to hertz. $\mathrm{s}(\mathrm{x})$ is the spin density.

Now let's consider applying a gradient $\mathrm{G}_{x}$ after the spins are flipped. $\mathrm{G}_{x}$ is a small field along the holding field direction but varies linearly in the transverse direction $\mathrm{x}$. Therefore, spins at different x positions experience a different additional field $G_{x} x$ and develop different additional phases according to $\gamma G_{x} x t$, where $\gamma$ is the gyromagnetic ratio of the nuclear species. As a result, Eq. 4.1 becomes:

$$
s(t)=C^{\prime} \int s(x) e^{i 2 \pi\left(f_{0} t+\gamma G_{x} x t\right)} d x
$$

In MRI we typically sample the demodulated signal. Using a reciprocal vector defined as $\vec{k}=\gamma \vec{G} t$, Eq. 4.2 can be rewritten as:

$$
s\left(k_{x}\right)=C^{\prime} \int s(x) e^{i 2 \pi k_{x} x} d x
$$

The vector $\vec{k}$ defines the so called "k space", and $\mathrm{s}(\mathrm{k})$ is often referred to as the "k-space data". Obviously, $\mathrm{s}\left(\mathrm{k}_{x}\right)$ and $\mathrm{s}(\mathrm{x})$ are a Fourier transform pair. The spin density $\mathrm{s}(\mathrm{x})$ can be determined by performing Fourier transform on $\mathrm{k}$-space data $\mathrm{s}\left(\mathrm{k}_{x}\right)$, which is called image reconstruction.

Eq. 4.3 can be easily extended to 2D:

$$
s\left(k_{x}, k_{y}\right)=C^{\prime} \int s(x, y) e^{i 2 \pi\left(k_{x} x+k_{y} y\right)} d x d y
$$


Similarly, the 2D spin density $\mathrm{s}(\mathrm{x}, \mathrm{y})$ can be reconstructed from $\mathrm{s}\left(\mathrm{k}_{x}, \mathrm{k}_{y}\right)$. In practice we can only sample discrete $\mathrm{k}$ space data. For $1 \mathrm{D}$ imaging we collect a vector and for the $2 \mathrm{D}$ case we sample a matrix. The image can then be reconstructed using discrete Fourier transform:

$$
s(m \Delta x, n \Delta y)=\frac{1}{N_{x} N_{y}} \sum_{p=0}^{N_{x}-1} \sum_{q=0}^{N_{y}-1} s\left(p \Delta k_{x}, q \Delta k_{y}\right) e^{2 \pi i \frac{m p}{N x}} e^{2 \pi i \frac{n q}{N y}},
$$

where $\mathrm{N}_{x}$ and $\mathrm{N}_{y}$ are the number of points sampled along the $\mathrm{k}_{x}$ or $\mathrm{k}_{y}$ directions. $\Delta x$ and $\Delta y$ are the spatial resolutions of the reconstructed image, which are determined by the range of $\mathrm{k}$ space covered by $\Delta x=\frac{1}{N_{x} \Delta k_{x}}, \Delta y=\frac{1}{N_{y} \Delta k_{y}}$.

From the discussions above, we can see that the key of MRI is to apply appropriate gradients and sample $\mathrm{k}$ space. The $\mathrm{k}$ space can be covered in many different ways. In our low field HP gas imaging experiment, we acquired $\mathrm{k}$ space data in either a line by line or point by point fashion, as described later in Section 4.4.1.

\subsection{Introduction of Low Field HP Gas MRI}

Magnetic resonance imaging of inhaled laser-polarized noble gases is a powerful technique for studying the ventilated gas space of the lung [4, 61, 62]. Historically, the lung has been a challenging target for MRI because of low tissue density as well as short T2* due to magnetic susceptibility differences at the countless air-tissue interfaces throughout the lung parenchyma. Using laser-based techniques, however, the spin- $1 / 2$ noble gases ${ }^{3} \mathrm{He}$ and ${ }^{129} \mathrm{Xe}$ can routinely be polarized to levels that are $10^{5}$ times higher than the thermal-equilibrium polarization at conventional MRI field strengths [63]. Laserpolarized noble gases are thus often said to be hyperpolarized. It is thereby possible to image the void spaces of the lung with spatial resolution on the order of $10 \mathrm{~mm}^{3}$ 61, 62. In addition, diffusion-weighted NMR of laser-polarized noble gases can be used to probe length scales much smaller than an imaging voxel, allowing one to characterize the size and geometry of the microscopic air spaces inside the lung and other porous structures [64, 65, 66, 67, 68].

Unlike conventional MRI, which strongly benefits from a high magnetic field, the signal-to-noise ratio (SNR) of MRI using laser-polarized noble gases is far less sensitive to the size of $B_{0}\left[69,70,71,72\right.$. Indeed, MRI of both hyperpolarized ${ }^{3} \mathrm{He}$ and ${ }^{129} \mathrm{Xe}$ has been reported at field strengths between 15 and $200 \mathrm{mT}$ using modified commercial 
scanners [73, 74, 75, 76]. Using largely homebuilt systems, hyperpolarized noble gas MRI has been demonstrated at field strengths as low as $2 \mathrm{mT}$ for ${ }^{3} \mathrm{He}$ [69, 177, 78, 79, 80, 81] and $34 \mathrm{mT}$ for ${ }^{129} \mathrm{Xe}$ [82].

There are several potential advantages to performing MRI at a low magnetic field [77. First, a low-field MRI system would be much less expensive and massive than a conventional high-field scanner, and could even be portable. It is also the case that local field variations due to magnetic susceptibility differences are greatly reduced at low field. Finally, low-field MRI is less sensitive to the presence of conducting materials such as metallic implants since the skin depth of radiofrequency (RF) electromagnetic waves is greater at lower frequencies [77]. Low-field noble-gas MRI thus has the potential to address specific niche applications where conventional high-field scanners would be unnecessary, impractical, or unfavorable.

Of the two widely used noble gases for hyperpolarized gas MRI, ${ }^{3} \mathrm{He}$ in general gives better image quality since it has a higher gyromagnetic ratio and is much easier to polarize. The availability of ${ }^{3} \mathrm{He}$, however, which in the United States comes largely from the decay of tritium associated with nuclear weapons, is severely limited. A recent increase in the demand for ${ }^{3} \mathrm{He}$ (mainly for use in neutron detectors) has resulted in sharp price increases [83]. On the other hand, ${ }^{129} \mathrm{Xe}$ is naturally abundant and can be extracted from the atmosphere. Also unlike ${ }^{3} \mathrm{He}$, xenon readily dissolves into tissue and blood, which makes studies of non-void spaces possible [84, 85, 86] and provides the opportunity to generate image contrast based on gas exchange in the lung [75, 87, 88, 89. In light of these factors, low-field imaging of laser-polarized ${ }^{129} \mathrm{Xe}$ is of particular interest going forward.

In this chapter, we describe a completely homebuilt apparatus for performing MRI of ${ }^{3} \mathrm{He}$ or ${ }^{129} \mathrm{Xe}$ at a very low magnetic field strength of $2 \mathrm{mT}$. To our knowledge, this is the lowest reported field strength for hyperpolarized ${ }^{129} \mathrm{Xe}$ MRI. The performance of our system is demonstrated by imaging laser-polarized phantoms containing either ${ }^{3} \mathrm{He}$ or ${ }^{129} \mathrm{Xe}$. In addition to conventional gradient-echo imaging, we also utilized a fully phase-encoded pulse sequence, which minimizes the undesirable effects of gas diffusion on image acquisition while making maximum use of the long $T_{2}^{*}$ decay time at very low fields. We also demonstrate the use of auxiliary measurements to further improve image quality, by characterizing and correcting for imperfections associated 
with our homebuilt MRI system. Finally, we discuss implications for in-vivo imaging of hyperpolarized noble gases using these techniques.

\subsection{Low Field HP Gas MRI Setup}

A schematic representation of our low-field MRI system is shown in Fig. 4.1. The system was controlled by an ordinary personal computer equipped with an input/output PCI card, which allowed flexible pulse sequences to be programmed and synchronized with data acquisition. A large Helmholtz coil provided a holding field, $\mathrm{B}_{0}$, of $2 \mathrm{mT}$, an intermediate-sized Helmholtz coil provided an excitation field, $\mathrm{B}_{1}$, at the ${ }^{129} \mathrm{Xe}$ resonant frequency, and two small detection coils were used to detect the precessing nuclear spins. Magnetic field gradients were generated by three pairs of Maxwell coils, which are omitted from Fig. 4.1 for clarity. All magnet coils were comprised of copper wire wrapped around rigid circular frames and were powered by off-the-shelf electronic components. The signal from the NMR pickup coils was amplified and processed using off-the-shelf analog hardware and then digitally sampled by the PCI card.

Our low field MRI scanner also incorporated a Spin-Exchange Optical Pumping (SEOP) setup, and the phantoms can be polarized and imaged without being physically moved. The phantoms were sealed glass cells containing laser polarized ${ }^{3} \mathrm{He}$ or

${ }^{129}$ Xe. A ceramic forced hot air oven (not shown in Fig. 4.1, described later) was used to maintain the phantoms at elevated temperatures needed to perform spin-exchange optical pumping. We note that many parts of our setup are also used for the development of polarized targets used in electron-scattering experiments [90]. Thus although some of the electronic components were over-specified for their purposes here, our MRI system was pieced together at minimal marginal cost.

\subsubsection{The Holding Field Coil, RF Coil, and Detection Coils}

In our MRI system the holding field $B_{0}$ and excitation field $B_{1}$ were both generated by Helmholtz coils. A Helmholtz coil consists of two identical circular loops of wire, both having the same number of turns and wound in series so that the same current flows in the same direction around each loop. The two loops are oriented parallel to one another along a common axis, and in the ideal case are separated by a distance $d$ equal to the radius $R$ of the circular loops. In this configuration, the spatial dependence of 


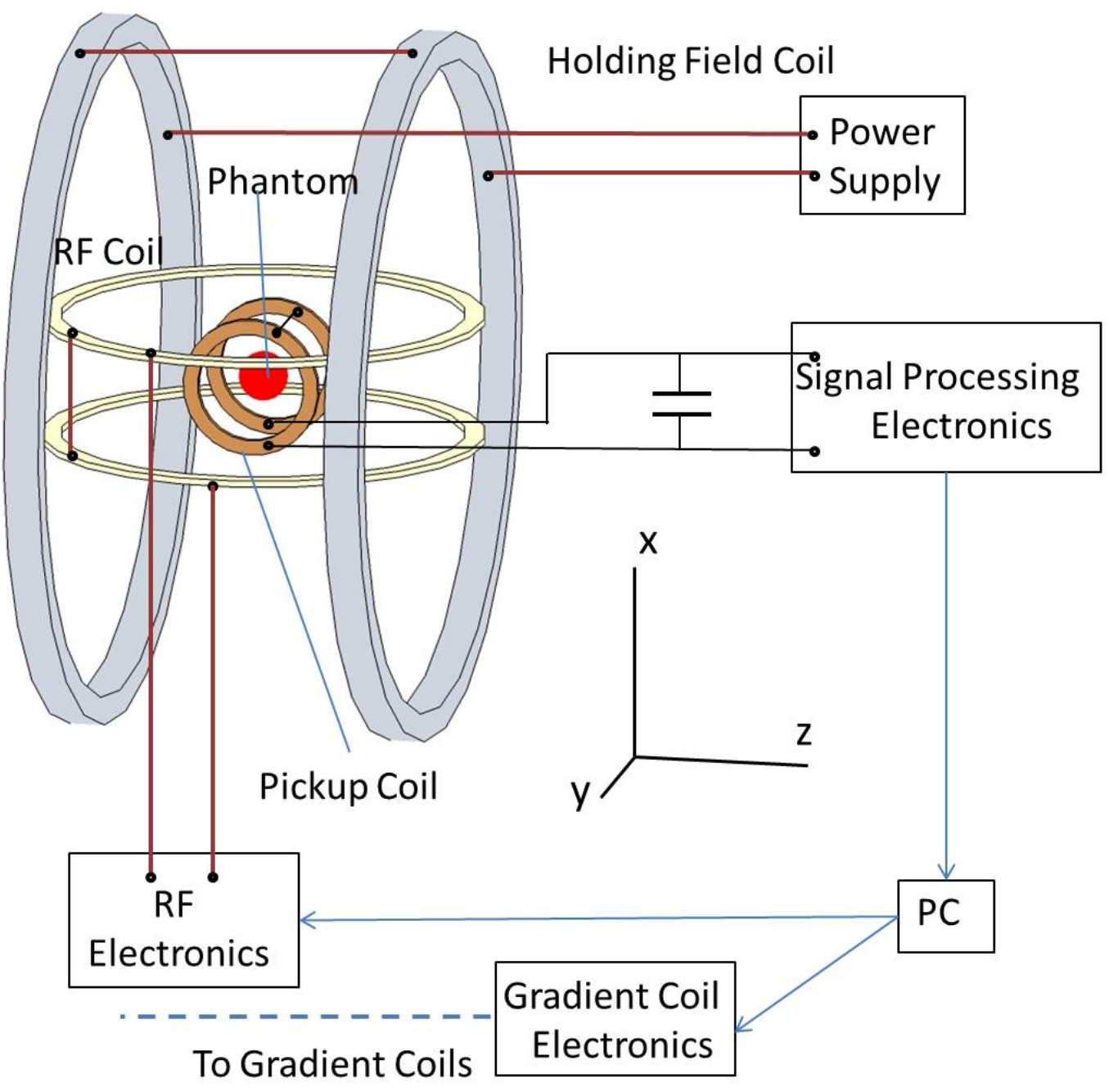

Figure 4.1: An overview of our low-field MRI apparatus. For clarity, the sizes of the $\mathrm{B}_{1}$ and pickup coils are exaggerated, and the gradient coils are omitted from this figure. The gradient coils are shown later in Fig. 4.2. A forced-air ceramic oven, also not shown here, encloses the pickup coils and phantom.

the magnetic field is zero through third order when expanded as a Taylor series in $z / R$, where $z$ is the distance from the midpoint, resulting in a highly uniform magnetic field near the center of the Helmholtz coil.

As shown in Fig. 4.1, the axis of the coil providing $B_{0}$ was horizontal and the axis of the coil providing $B_{1}$ was vertical. Both pairs of loops were placed symmetrically about the location of the phantom, represented by the red sphere in Fig. 4.1. Each loop 
of the $B_{0}$ coil consisted of 240 turns of gauge 6 square magnet wire, with $\mathrm{d}=\mathrm{R}=66 \mathrm{~cm}$. Direct current (6.8 A for a $2 \mathrm{mT}$ magnetic field) was provided by a current-regulated DC power supply (HA-1365-3SS, Walker LDJ Scientific, Auburn Hills, MI).

In most MRI systems the $B_{0}$ field is provided by a solenoid-style electromagnet, which delivers relatively high magnetic field uniformity and power efficiency in a compact geometry. By comparison, a Helmholtz coil must be significantly larger and requires higher current to achieve similar levels of spatial uniformity and field strength. A large Helmholtz coil was well suited for our system, however, despite the lower power efficiency, since high field strength is not a priority and the open geometry provided plenty of space to accommodate our Maxwell-style transverse gradient coils while also allowing easy access to the imaged subject. In our setup, the calculated $B_{0}$ field (taking into account the finite thickness of the wire bundle forming each loop) varied less than 7 parts-per-million (ppm) over the central $5 \mathrm{~cm} \times 5 \mathrm{~cm} \times 5 \mathrm{~cm}$ volume, which constituted a "sweet spot" of suitable size to accommodate our phantoms or rodent lungs.

Each loop of our $\mathrm{B}_{1}$ coil consisted of 13 turns of gauge 16 magnet wire wrapped around $24.1 \mathrm{~cm}$ radius circular frames, with a center-to-center separation $d=24.6 \mathrm{~cm}$ along the vertical coil axis. The sinusoidal RF drive signal (65 kHz or $26 \mathrm{kHz}$, depending on the nucleus being studied) was provided by a function generator (33250A, Agilent Technologies, Santa Clara, CA) and amplified by a low-frequency RF power amplifier (ENI 1040L, Electronic Navigation Industries, Rochester, NY).

The NMR signal was detected by a pair of much smaller circular coils placed on either side of the imaged subject. These coils were oriented perpendicular to the $B_{0}$ and $B_{1}$ coils, as shown in Fig. 4.1. Each pick-up coil consisted of 90 turns of gauge 27 magnet wire, wound in series around circular frames of radius $1.5 \mathrm{~cm}$ and separated by $4.3 \mathrm{~cm}$. The resonant frequency of the detection circuit was tuned to the Larmor frequency of either ${ }^{3} \mathrm{He}$ or ${ }^{129} \mathrm{Xe}$ by placing an interchangeable capacitor across the inputs of the pre-amplfier. Further details of the transmit/receive RF chain are provided in Section 4.3 .3

\subsubsection{The Gradient Coils}

The simplest way to generate a magnetic field gradient along the same direction as the $B_{0}$ holding field is to use a so-called Maxwell coil, which consists of two oppositely wound but otherwise identical circular loops of wire separated by a distance $d$ along a 
common axis. This arrangement is similar to the Helmholtz configuration, except the currents in the two loops flow in opposite directions and the optimum separation is given by $d=\sqrt{3} R$, which is larger than the optimum separation for a Helmholtz coil. At this distance, the leading nonlinear term in the Taylor expansion of the resulting magnetic field is fifth order in $z / R$, leaving a highly uniform longitudinal gradient near the center of the Maxwell coil. Previously described low-field systems have used either this exact arrangement [69] or the same basic arrangement but with additional coaxial loops to further expand the region of uniformity [81].

Generating a magnetic field gradient along a direction perpendicular to the $B_{0}$ holding field is more complicated, as the gradient coil must produce a $B_{z}$ component that varies in strength along the perpendicular direction. In both low- and high-field scanners, the magnetic field gradients along the transverse directions are typically produced using some variant of Golay coils, which in their simplest form are saddle shaped and can be wound on the surface of a cylinder. This geometry is convenient for fitting inside a cylindrical $B_{0}$ magnet [91]. In our system, which did not have the same geometric constraints due to the use of large Helmholtz coils for the $B_{0}$ and $B_{1}$ fields, we not only used a Maxwell coil to provide the longitudinal gradient $G_{z}$, but also generated transverse gradients $G_{x}$ and $G_{y}$ by using two additional Maxwell coils oriented at so-called "magic angles", as shown in (Fig. 4.2). The idea of using such an arrangement was discussed by Cates et al. for a different application in [41]. However, to our knowledge this is the first time that transverse imaging gradients have been provided by this type of coil.

The proper orientation of the transverse-gradient Maxwell coils can be derived from the gradient tensor as follows. The $x-y$ - $z$ coordinate system is defined such that the $z$ axis points in the longitudinal direction (the same direction as the $B_{0}$ field) and the $x$ and $y$ axes lie in the transverse plane. Within this coordinate system, the direction of the coil axis is described by the polar coordinates $\theta$ and $\phi$, where $\theta$ is the angle with respect to the $\mathrm{z}$ axis, and $\phi$ is the azimuthal angle made by the projection of the coil axis onto the $x-y$ plane, as shown in Fig. 4.2(a). The components of the gradient tensor that are most important for imaging are $\partial B_{z} / \partial x, \partial B_{z} / \partial y$, and $\partial B_{z} / \partial z$, corresponding to the $x, y$, and $z$ gradients of the $z$-component of the holding field $\left(B_{z}\right)$, which are often referred to as $G_{x}, G_{y}$, and $G_{z}$ respectively. Here we ignore the effect of $B_{x}$ and $B_{y}$ field gradients, although they can in principle cause small distortions of the image. At the center of a Maxwell coil, these components of the gradient tensor are given by 


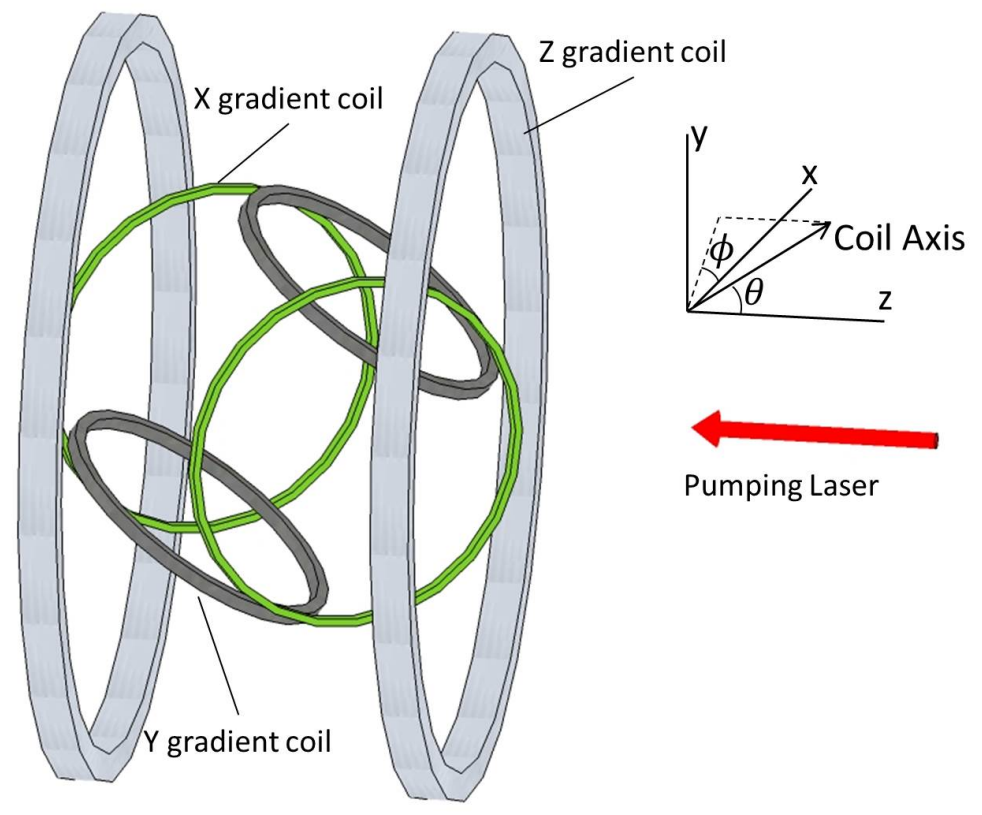

(a) Overview of the gradient coils
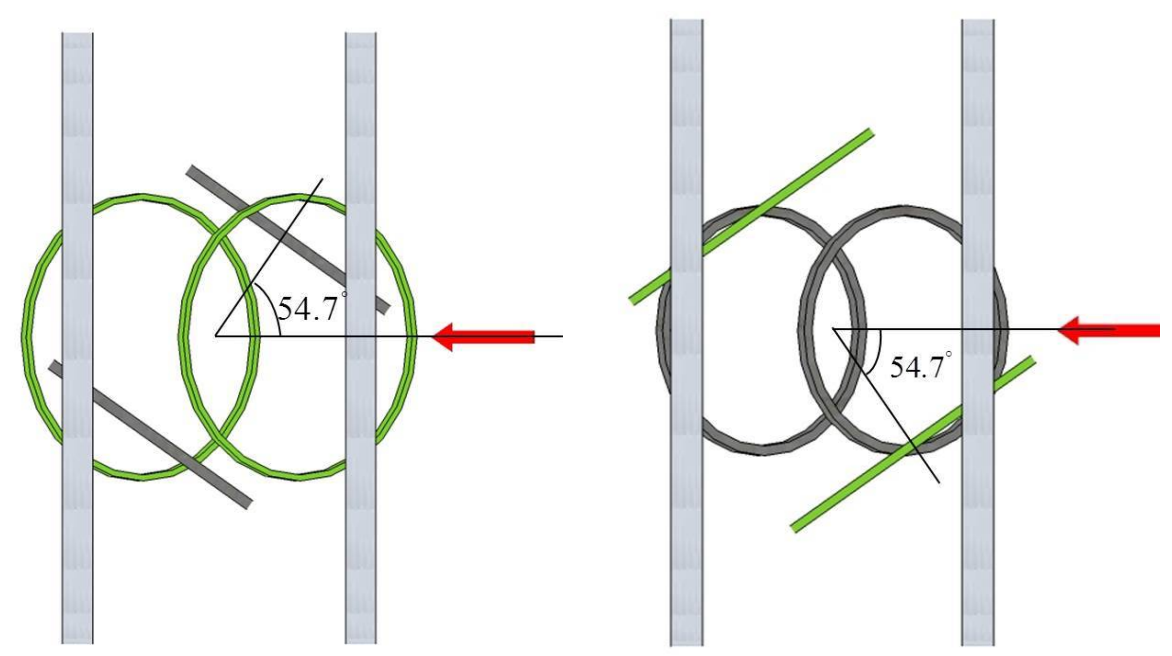

(b) Left view (along $\mathrm{x}$ direction) of the (c) Top view (along $\mathrm{y}$ direction) of the gragradient coils dient coils

Figure 4.2: Observing the Gradient coils from different angles.

[41]:

$$
\nabla \mathbf{B}(\theta, \phi)=\left(\begin{array}{lll}
\partial B_{x} / \partial x & \partial B_{y} / \partial x & \partial B_{z} / \partial x \\
\partial B_{x} / \partial y & \partial B_{y} / \partial y & \partial B_{z} / \partial y \\
\partial B_{x} / \partial z & \partial B_{y} / \partial z & \partial B_{z} / \partial z
\end{array}\right)
$$




$$
=3 \kappa I\left(\begin{array}{ccc}
\sin ^{2}(\theta) \cos ^{2}(\phi)-1 / 3 & \sin ^{2}(\theta) \sin (\phi) \cos (\phi) & \sin (\theta) \cos (\theta) \cos (\phi) \\
\sin ^{2}(\theta) \sin (\phi) \cos (\phi) & \sin ^{2}(\theta) \sin ^{2}(\phi)-1 / 3 & \sin (\theta) \cos (\theta) \sin (\phi) \\
\sin (\theta) \cos (\theta) \cos (\phi) & \sin (\theta) \cos (\theta) \sin (\phi) & \cos ^{2}(\theta)-1 / 3
\end{array}\right)
$$

where

$$
\kappa=\frac{3 \pi n R^{2} d}{10\left(d^{2} / 4+R^{2}\right)^{5 / 2}}\left[\mathrm{G}(\mathrm{cm} \mathrm{A})^{-1}\right]
$$

$n$ is the number of turns and $I$ is the current.

The "magic angle" $\theta_{m}$ is the angle for which $\partial B_{z} / \partial z=0$. At this angle, given by $\theta_{m}=\arccos (1 / \sqrt{3})=54.7^{\circ}$, the contribution to $G_{z}$ disappears, and the coil generates a purely transverse $B_{z}$ gradient. The azimuthal angle $\phi$ can then be chosen to make either $\partial B_{z} / \partial x$ or $\partial B_{z} / \partial y$ zero.

If $\theta=\theta_{m}$ and $\phi=0$,

$$
\nabla B\left(\theta_{m}, 0\right)=\kappa I\left(\begin{array}{ccc}
1 & 0 & \sqrt{2} \\
0 & -1 & 0 \\
\sqrt{2} & 0 & 0
\end{array}\right)
$$

In this case we have $\partial B_{z} / \partial x=\sqrt{2} \kappa I$, and $\partial B_{z} / \partial y=\partial B_{z} / \partial z=0$. We will refer to $\partial B_{z} / \partial x$ as the $\mathrm{x}$ gradient $\left(G_{x}\right)$.

Similarly, if $\theta=\theta_{m}$ and $\phi=\pi / 2$,

$$
\nabla B\left(\theta_{m}, \pi / 2\right)=\kappa I\left(\begin{array}{ccc}
-1 & 0 & 0 \\
0 & 1 & \sqrt{2} \\
0 & \sqrt{2} & 0
\end{array}\right)
$$

and we have $\partial B_{z} / \partial y=\sqrt{2} \kappa I$ and $\partial B_{z} / \partial x=\partial B_{z} / \partial z=0$. We will refer to $\partial B_{z} / \partial y$ as the y gradient $\left(G_{y}\right)$.

The more commonly used longitudinal Maxwell pair has $\theta=0$ and $\phi=0$. Its gradient tensor is:

$$
\nabla B(0,0)=\kappa I\left(\begin{array}{ccc}
-1 & 0 & 0 \\
0 & -1 & 0 \\
0 & 0 & 2
\end{array}\right)
$$

Both $\mathrm{x}$ and $\mathrm{y}$ gradients are zero. The only important nonzero term is $\partial B_{z} / \partial z=2 \kappa I$, which we will call the z gradient $\left(G_{z}\right)$.

Thus by using three Maxwell coils in such an arrangement we can independently control the $\mathrm{x}, \mathrm{y}$, and $\mathrm{z}$ gradients, which is highly desirable for implementing an MRI pulse sequence. 
In our system, the $x, y$, and $z$ gradient coils had 42, 100 and 8 turns of wire, respectively. The corresponding radii were $33 \mathrm{~cm}, 28 \mathrm{~cm}$ and $66 \mathrm{~cm}$, and the separations between the two loops of each Maxwell pair were $64 \mathrm{~cm}, 56 \mathrm{~cm}$ and $66 \mathrm{~cm}$. Note that they were not placed in the optimum separation $d=\sqrt{3} R$ due to spatial constraints. Fig. 4.3 shows the theoretical performance of our $y$ gradient coil. These plots were generated numerically in MATLAB (MathWorks, Natick, MA) by adding the magnetic field generated by two current loops with specified radius and separation [57]. These results thus take into account the non-ideal geometry of the $y$-gradient coil. Fig. 4.3(a) shows the $z$ component of the magnetic field $\left(B_{z}\right)$ due to this coil, computed over the central region of the $x-y$ plane. $B_{z}$ appears to vary linearly with $y$, as desired. The slope of this curve along the $y$ direction, which is equal to the $y$ gradient $G_{y}$, is shown in Fig. 4.3(b), The gradient nonlinearity is less than $0.4 \%$ over the central $5 \mathrm{~cm}$ by $5 \mathrm{~cm}$ region of the transverse plane. If the optimum loop separation were used, the theoretical nonlinearity would be better than $0.03 \%$ over this same region.

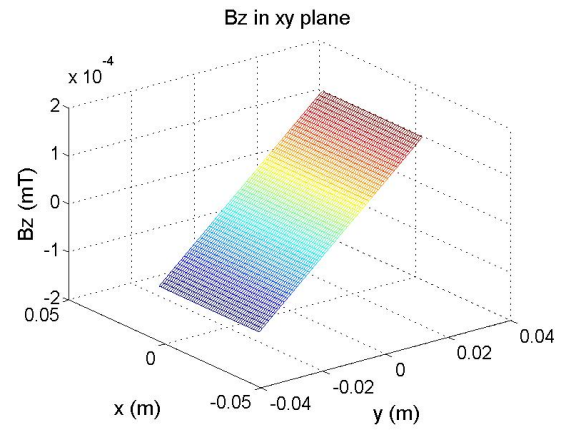

(a) $B_{z}$ in XY plane.

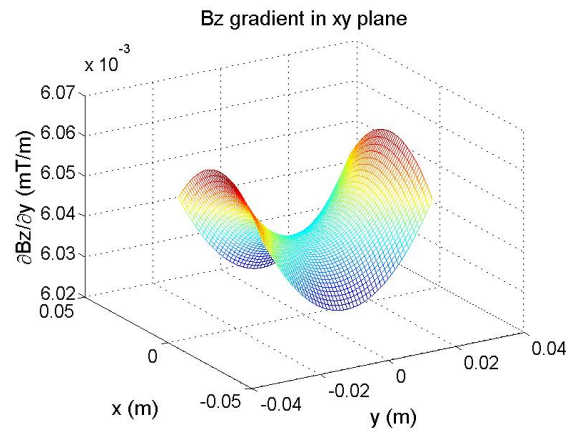

(b) $B_{z}$ gradient in XY plane.

Figure 4.3: $B_{z}$ and its gradient generated by the actual y gradient coils per Ampere per turn in the x-y plane.

The $x$ and $y$ gradient coils were powered by high speed, bipolar operational power amplifiers (BOP 72-6M and BOP 36-12M, Kepco, Flushing, NY), which provided maximum currents of $12 \mathrm{~A}$ and $6 \mathrm{~A}$, respectively. At these currents, the achievable gradient strength were limited to $\sim 3.9 \mathrm{mT} / \mathrm{m}$ in the $x$ direction and $\sim 3.6 \mathrm{mT} / \mathrm{m}$ in the $y$ direction. The $z$ gradient coil was powered by one output channel of a triple-output DC power supply (1760 DC power supply, BK precision, Yorba Linda, CA). For the imaging studies presented here, only the $x$ and $y$ gradient coils were used for spatial 
encoding. However, all three gradient coils were used to shim the $B_{0}$ holding field, as described later in Section 4.3.5,

\subsubsection{Control and Data Acquisition Electronics}

Fig. 4.4 illustrates the architecture of the electronics used to control our MRI system. The heart of the electronic control system was an ordinary personal computer equipped with a multifunction data acquisition card (PCI-6259, National Instruments Corporation, Austin, TX). Input/output functionality was programmed in LabVIEW (National Instruments Corporation, Austin, TX), which allowed flexible pulse sequences to be executed. Two of the analog outputs provided control voltages for the $x$ and $y$ gradient power supplies, a third analog output was used to generate a digital gating pulse for synchronizing RF transmit and data acquisition, and two analog input channels were used to digitize and record the real and imaginary parts of the NMR signal from the RF receive chain. The digital gating pulse was sent to the RF function generator and determined the start time and duration of the transmit RF pulse. The signal from the detection circuit was first band-pass filtered and amplified by an SR560 low-noise preamplifier, and then sent to an isolation switch (ZYSWA-2-50DR, MiniCircuits, Brooklyn, NY) which was also controlled by the digital gating pulse. This switch isolated the detection coil circuit from the downstream RF electronics during RF transmit. When RF was not transmitting, the signal passed through to the next stage, where it was split in two and mixed with two different reference signals, each produced by a dedicated function generator (33250A, Agilent Technologies, Santa Clara, CA). Both reference signals were set to the same frequency (equal to the nominal Larmor frequency of the nucleus being imaged) but differed by a $90^{\circ}$ phase shift, to generate the real and imaginary parts of the NMR signal. Alternatively, the reference signals can be generated by a single function generator and a $90^{\circ}$ phase shifter. Finally, the outputs of the mixer circuits were low-pass filtered and further amplified by two additional SR560 preamplifiers, and the resulting demodulated NMR signals were digitized by 16-bit analog-to-digital converters on the data acquisition card.

\subsubsection{Laser-Polarized Phantoms}

We imaged two phantoms. They were filled with $2.8 \mathrm{~atm}$ of either ${ }^{3} \mathrm{He}$ or isotopically enriched Xe $\left(86.2 \%{ }^{129} \mathrm{Xe}\right)$, a small amount of $\mathrm{N}_{2}(\sim 200$ Torr $)$ and several droplets of 


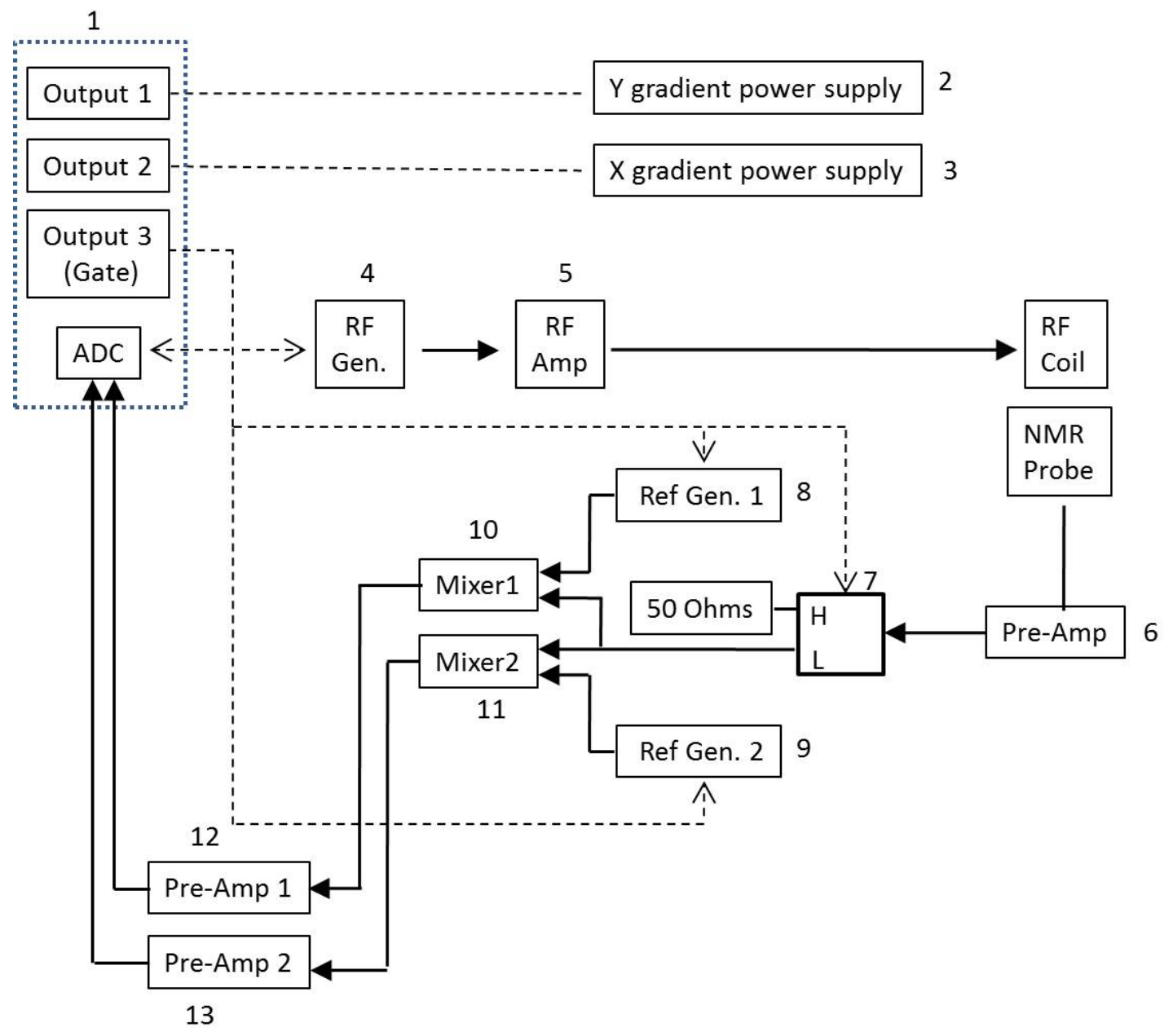

Figure 4.4: Low field imaging system schematic. The part numbers for each component are as follows: 1, PC with NI DAQ 6259 card; 2 and 3, Kepco BOP 72-6M and 36-12M power supplies; 4, 8 and 9, Agilent 33250A Function Generators; 5, ENI Model 1040L power amplifier; 6, 12 and 13, SR560 low noise preamplifier ; 7, Mini-Circuits ZYSWA-2-50DR Isolation Switch; 10 and 11, Mini-Circuits ZAD-8 frequency mixers.

Rb. The phantom containing ${ }^{3} \mathrm{He}$ was spherical in shape as shown in Fig. 4.5(a), The phantom containing ${ }^{129} \mathrm{Xe}$ was shaped like the letter " $\mathrm{X}$ ", and is shown in Fig. 4.5(b). The phantoms were polarized using the technique of Spin-Exchange Optical Pumping (SEOP). The phantoms were illuminated by about $30 \mathrm{~W}$ of spectrally-narrowed laser power with a line width of $0.2 \mathrm{~nm}$ FWHM. For our ${ }^{3} \mathrm{He}$ phantom, SEOP was performed at $175^{\circ} \mathrm{C}$. In the case of our ${ }^{129} \mathrm{Xe}$ phantom, the optimum oven temperature was about 

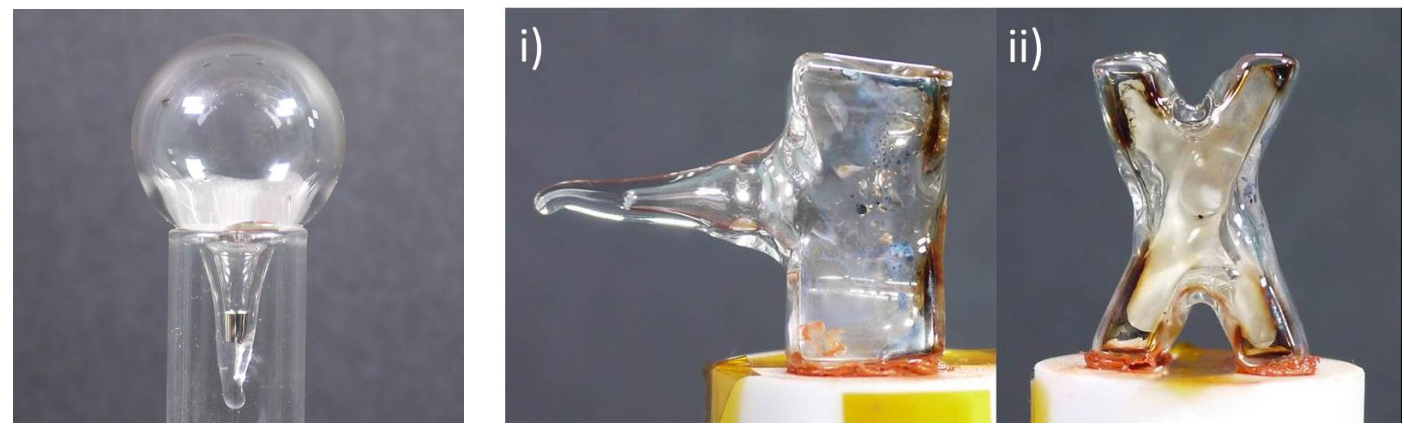

(a) The spherical ${ }^{3} \mathrm{He}$ phantom. (b) Side and front view of the Xenon phantom. The dimension of The diameter is about 1". the phantom is $1.5 \mathrm{~cm}$ by $2.5 \mathrm{~cm}$.

Figure 4.5: Phantoms that we imaged in our low field scanner.

$85^{\circ} \mathrm{C}$. Note that these temperatures were measured in the heated oven but not inside the phantoms. The phantom temperatures could be tens of degrees higher due to laser heating.

Under the aforementioned conditions, the ${ }^{3} \mathrm{He}$ polarization built up with a time constant of 6 hours and the saturation polarization was about $40 \%$. The ${ }^{129}$ Xe polarization build-up time constant was about $45 \mathrm{~s}$ and the polarization saturated at only

$6 \%$. Although our Xe polarization was fairly low, much higher ${ }^{129}$ Xe polarizations can be achieved in the flowing-xenon polarizers used for medical imaging [11, 92, 93]. Therefore the ${ }^{129} \mathrm{Xe}$ polarization available for in-vivo applications of our MRI system can be much higher than we achieved in our sealed xenon phantom.

\subsubsection{Field Shimming and Transmitter Calibration}

All three gradient coils were used to shim the $B_{0}$ holding field. Thus in principle, the linear component of any field inhomogeneities at the isocenter of our MRI system could be completely canceled. Shimming was performed empirically, by manually adjusting the current in each of the three gradient coils to maximize the duration of the free induction decay (FID) following a nonselective RF excitation of the laser-polarized phantom. The required shim currents were then implemented as constant offsets on the output of the gradient power supplies.

Fig. 4.6 shows a representative FID signal from the ${ }^{129} \mathrm{Xe}$ phantom at $\sim 2 \mathrm{mT}$. The decay time constant $\left(T_{2}^{*}\right)$ is $3.2 \mathrm{~s}$. Such a long lasting FID is one of the special 
characteristics of low field operation. By contrast, the $T_{2}^{*}$ observed in laser-polarized ${ }^{3}$ He phantoms at high field $(1.5 \mathrm{~T})$ has been measured to be less than $100 \mathrm{~ms}$ in a spherical phantom in a well-shimmed field [94].

Transmitter flip-angle calibration was performed by taking a series of identical FID measurements in a time period that was short compared to spin exchange and all other relaxation rates. The flip angle $\theta$ of the $\mathrm{RF}$ pulse was determined by fitting the resulting signal amplitudes $S_{n}$ to the sequence $S_{n}=S_{0}(\cos \theta)^{(n-1)} \sin \theta$.

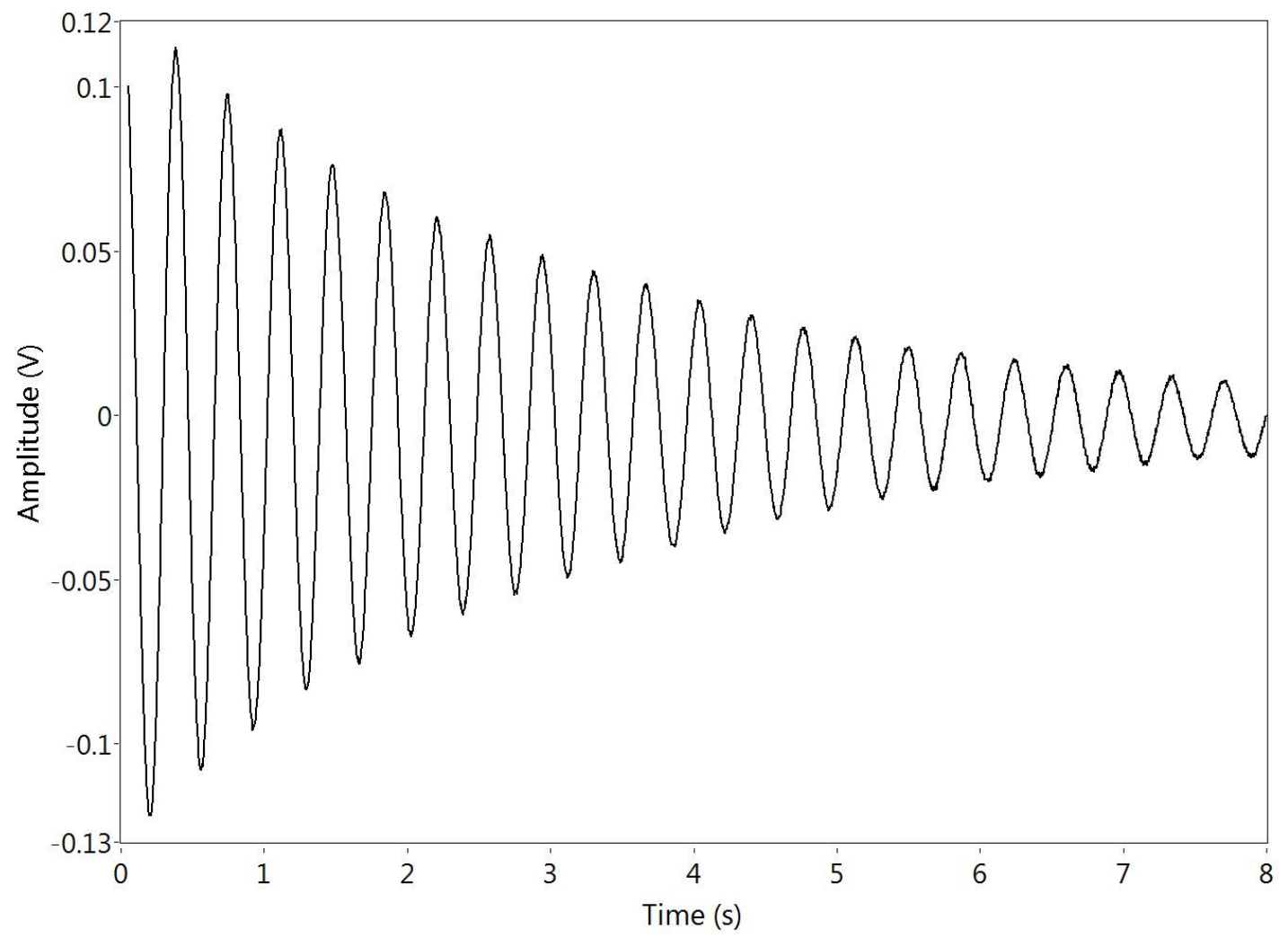

Figure 4.6: A FID with the shimmed holding field. The decay time constant $\left(T_{2}^{*}\right)$ is about $3.2 \mathrm{~s}$. In comparison, $T_{2}^{*}$ is less than $200 \mathrm{~ms}$ without the shimming gradients.

\subsection{Imaging Methods}

\subsubsection{Pulse Sequences}

In the work presented here, we used several different pulse sequence strategies depending on the particular study. For imaging of the ${ }^{3} \mathrm{He}$ phantom, which has a magnetic moment 
$\sim 3$ times larger than that of ${ }^{129} \mathrm{Xe}$ and is easier to polarize, we used a small tip angle gradient echo pulse sequence (Fig. 4.7), which is similar to a Fast Low Angle SHot (FLASH) sequence except that no spoiling is used. Instead, we waited long enough after each excitation for the transverse magnetization to decay away before sending the next pulse. An image of the phantom was acquired after a single "cycle" of laser polarization, i.e., the ${ }^{3} \mathrm{He}$ gas was polarized using SEOP, and all imaging information was acquired using the available magnetization without ever re-polarizing the gas. In the case of ${ }^{129} \mathrm{Xe}$, the NMR signal size was much smaller, which made it difficult to collect a high quality image with one cycle of SEOP. However, ${ }^{129}$ Xe polarization built up relatively fast (time constant $\sim 45 \mathrm{~s}$ under our SEOP conditions), and we were able to acquire imaging information over multiple laser-polarization cycles. In one set of studies we used the gradient echo pulse sequence, and the ${ }^{129} \mathrm{Xe}$ gas was repolarized after acquiring a single line of $\mathrm{k}$ space. In another set of studies, we applied a fully phase encoded pulse sequence, and Xe was repolarized after acquiring each point in $\mathrm{k}$ space. For both ${ }^{3} \mathrm{He}$ and ${ }^{129} \mathrm{Xe}$, a nonselective excitation RF pulse of $400 \mu$ s duration was applied, yielding a projection image in the $x-y$ plane.

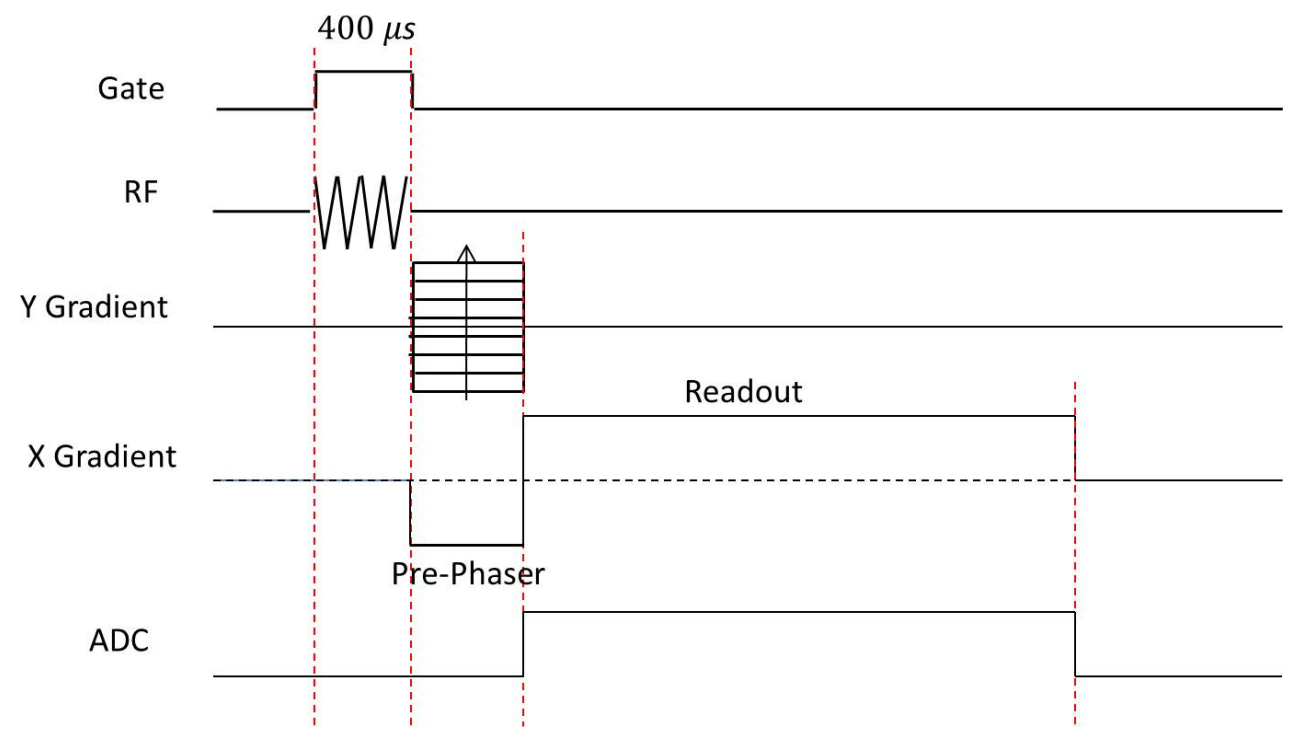

Figure 4.7: A Typical gradient echo pulse sequence. For the ${ }^{3} \mathrm{He}$ phantom, y gradient and the pre-phasing lobe was applied for $7 \mathrm{~ms}$, and the readout gradient and $\mathrm{ADC}$ was on for $36 \mathrm{~ms}$. In the case of our xenon phantom, these two durations were $20 \mathrm{~ms}$ and $70 \mathrm{~ms}$ respectively. 
The gradient-echo acquisitions were performed in a conventional manner, in which a single line of the two-dimensional Cartesian $k$ space was acquired following each excitation RF pulse. Frequency encoding was applied in the physical $x$ direction using a gradient amplitude of $G_{x}=0.85 \mathrm{mT} / \mathrm{m}$, and phase encoding was applied in the physical $y$ direction by varying the amplitude of $G_{y}$ (Fig. 4.7). Thirty-two lines of $k$ space were acquired in sequential order, with the seventeenth line corresponding to $k_{y}=0$. The field of view for both noble gases was $32 \mathrm{~mm}$ and the in-plane resolution was $1 \mathrm{~mm}$. For ${ }^{3} \mathrm{He}$, we used a tip angle of $\sim 14^{\circ}$ and a repetition time of $2 \mathrm{~s}$, much shorter than the spin-up time constant. The total time required for imaging was thus about $1 \mathrm{~min}$. On the other hand, the saturation magnetization was much lower in the Xe phantom. We used a large flip angle $\left(90^{\circ}\right)$ and long repetition time $(\mathrm{TR}=1$ min, which was $\sim 1.4$ polarization time constants), which allowed the hyperpolarized xenon magnetization to largely regrow between excitation RF pulses. Therefore the time needed to acquire all information for an image was about $0.5 \mathrm{hrs}$, even though the actual data acquisition time was only $3 \mathrm{~s}$. The pulse sequence was then repeated and a total of 9 Xe images were acquired.

In our gradient echo pulse sequences, readout was performed asymmetrically with respect to $k_{x}=0$. For ${ }^{3} \mathrm{He}$, the duration of the phase-encoding gradient and readout pre-phaser were both $7 \mathrm{~ms}$, and the duration of the readout gradient was $25 \mathrm{~ms}$. For ${ }^{129} \mathrm{Xe}$ these two durations were $20 \mathrm{~ms}$ and $70 \mathrm{~ms}$ respectively. Asymmetric readout allowed the echo time to be reduced and therefore suppressing diffusion-induced attenuation of the frequency-encoded signal. Taking Xe MRI for example, the echo time was reduced by the asymmetric acquisition from $100 \mathrm{~ms}$ to $40 \mathrm{~ms}$, which lowered the corresponding $b$ value (which relates the diffusion attenuation to diffusion coefficient by $S=S_{0} e^{-b D}$ ) from $33 \mathrm{~s} / \mathrm{cm}^{2}$ to $2 \mathrm{~s} / \mathrm{cm}^{2}$ while maintaining a maximum $k_{x}$ value corresponding to $1 \mathrm{~mm}$ spatial resolution.

In the fully phase-encoded acquisition, data were acquired on a symmetric $64 \times 64$ $k$-space matrix corresponding to $32 \mathrm{~mm}$ imaging field of view and $0.5 \mathrm{~mm}$ in-plane spatial resolution. A single point of the full $k$-space matrix was sampled following each excitation RF pulse, by opening up the readout ADC immediately following the application of phase-encoding gradient pulses along both $x$ and $y$ axes, as shown in Fig. 4.8. Because only one point in $k$ space was acquired after each RF excitation, 4096 excitations were required to sample the entire $64 \times 64$ matrix. We chose a compromise 
between the tip angle and $T_{R}$ to get a reasonable signal size while keeping the whole MRI procedure short enough to be practical. Our tip angle was $35^{\circ}$ and $T_{R}$ was $18 \mathrm{~s}$. As a result, it took $\sim 20$ hours to collect all the data, and the ADC was open for $\sim 7 \mathrm{hrs}$ in total.

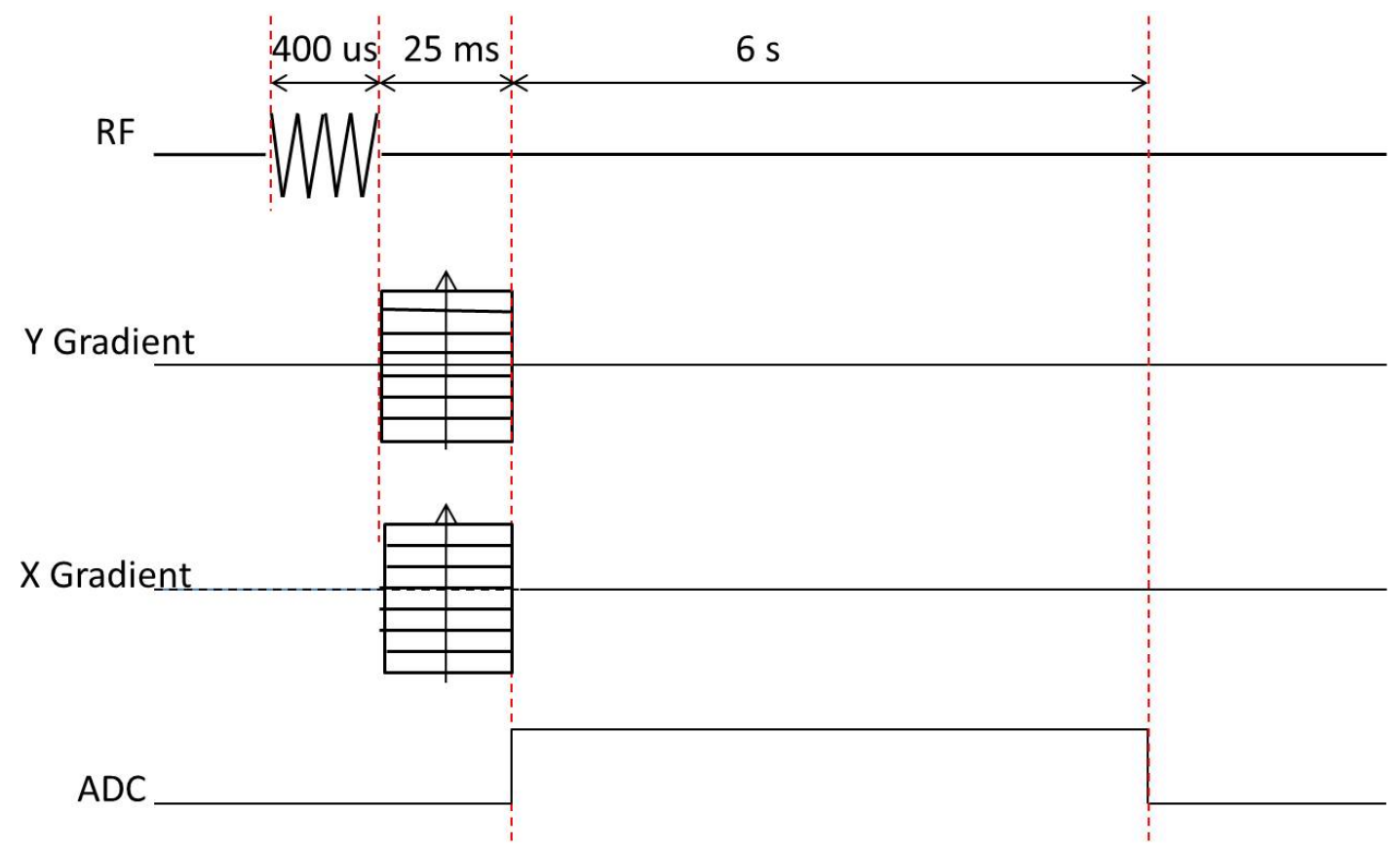

Figure 4.8: Fully phase encoded pulse sequence. The time axis is not to scale. The phase-encoding gradient waveforms are depicted as square here, but actually have the shape depicted in Fig. 4.10 .

\subsubsection{Auxiliary Measurements}

To better understand the imperfections of our homebuilt MRI system, and to investigate the possibility of improving low-field image quality by correcting for these imperfections during image reconstruction, we performed several auxiliary measurements of system performance in conjunction with the imaging pulse sequences described in Section 4.4.1.

Our $B_{0}$ Helmholtz coil was not magnetically shielded, nor was the magnetic field itself actively regulated. In such a system, it is common for the magnetic field to change over time due to various factors such as power supply output drift, the movement of nearby ferromagnetic objects, or stray magnetic fields generated by other sources. In their low-field imaging studies of laser-polarized ${ }^{3} \mathrm{He}$, Wong et al. observed $B_{0}$ field 


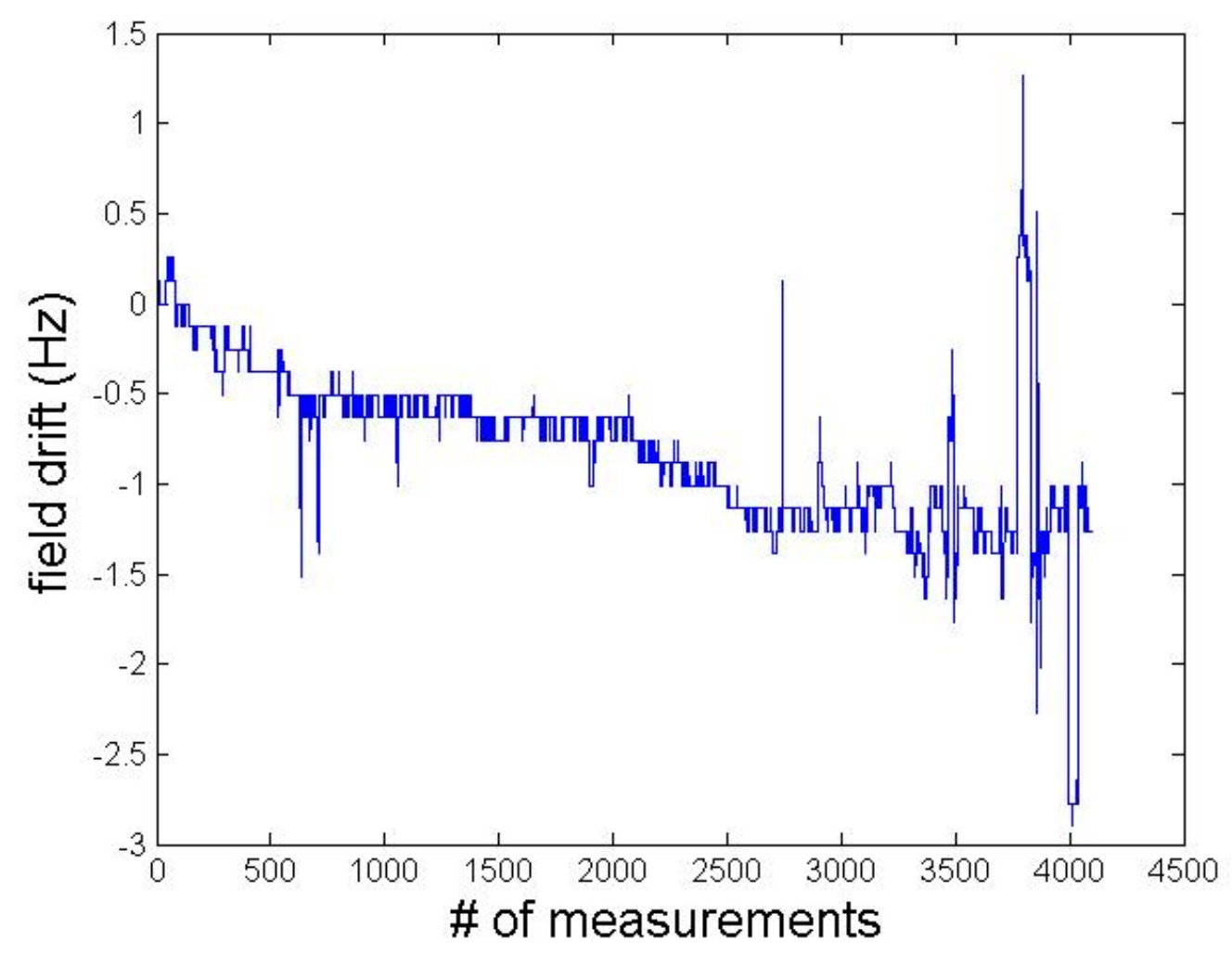

Figure 4.9: Holding field drift in ${ }^{129}$ Xe precession frequency during a fully phase encoded imaging procedure.

changes on the order of $100 \mathrm{ppm}$ over several hours [69]. It was concluded that this level of field drift was inconsequential for their purposes, since the changes were slow compared with the time required for image acquisition. A similar level of field drift could be relevant to our imaging studies, however, since our images were acquired over much longer periods of time.

Therefore we endeavored to measure $B_{0}$ field changes directly at the location of the phantom, by periodically acquiring un-encoded free-induction decays following a small-tip-angle excitation, to monitor the resonant frequency of the ${ }^{129} \mathrm{Xe}$ spins. The extremely long $T_{2}^{*}$ allowed us to resolve ten-ppm level magnetic field changes using this method. For the point-by-point acquisition, the acquisition of each phase encoded $k$-space point was immediately preceded by an NMR measurement of the resonant frequency using a $5^{\circ}$ flip angle. Fig. 4.9 shows the measured field drift throughout the 
entire acquisition. We suspect that the sudden changes were caused by magnetic fields generated by equipments nearby.

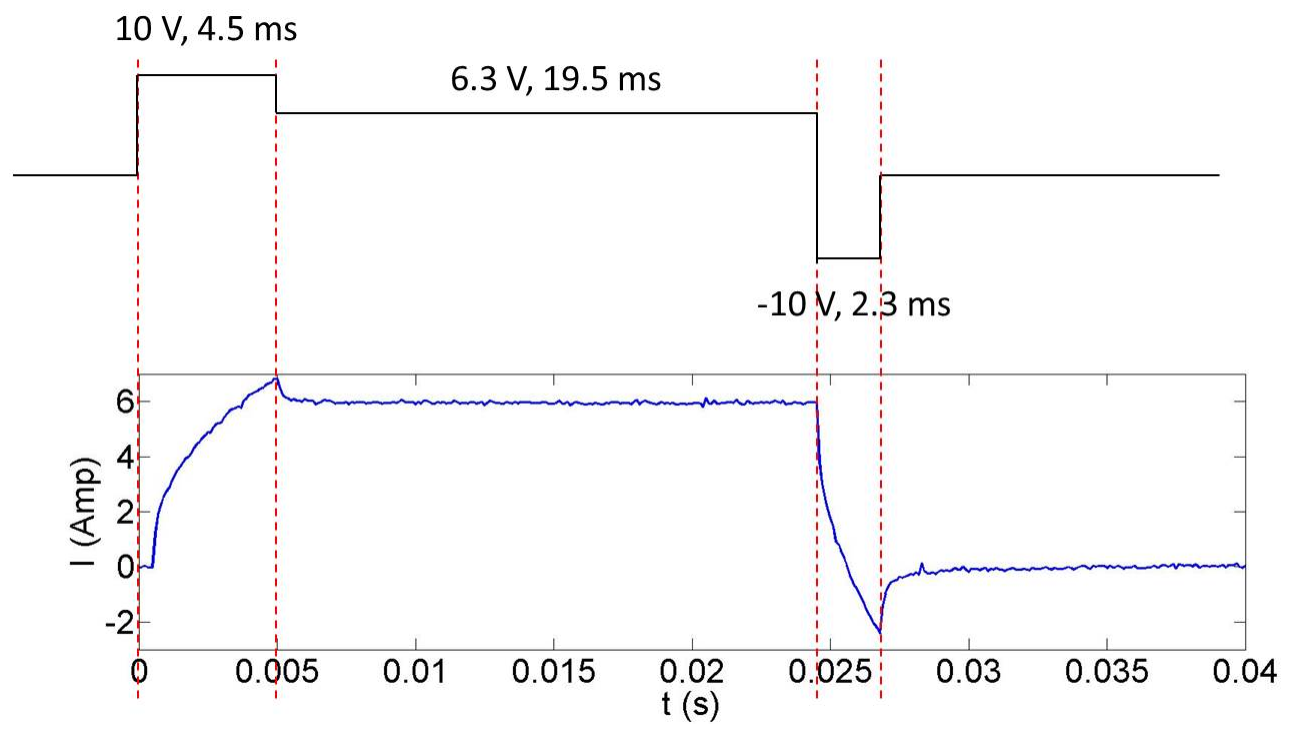

Figure 4.10: Control voltage and the current response of our $y$ gradient coil for the maximum phase-encoding gradient. The pre- and post- emphasis pulses help the current to reach an equilibrium state faster.

Another potential source of error is gradient infidelity. We assessed gradient fidelity by connecting a small shunt resistor (Voltmeter Current Shunt CS-10, Isotek Corporation, Swansea, MA) in series with the $y$ gradient coil leads and recording the voltage across it while the imaging gradients were applied, using one of the spare analog inputs on the DAQ card. The coil current (and thus the gradient amplitude) was directly proportional to this voltage. The first thing we noticed was a long gradient rise time despite square-wave input. To compensate for this, we added pre- and post-emphasis pulses to our gradient coil control voltages as shown in Fig. 4.10, to accelerate the effective ramp times.

We also used the shunt resistor to measure the phase-encoding gradient moments, by integrating the measured coil current. The $k$-space coordinates of the sampled $k$ space locations were proportional to these moments. For the fully phase-encoded imaging sequence, there was noticeable nonlinearity of the $k_{y}$ trajectory (Fig. 4.11), especially near $k_{y}=0$. We speculate that this peculiarity originated from the bipolar nature of the gradient power supply, which abruptly switches transistor banks when the output 
voltage swings from positive to negative. We also measured the $k_{x}$ trajectory, but found no obvious deviation from linearity.

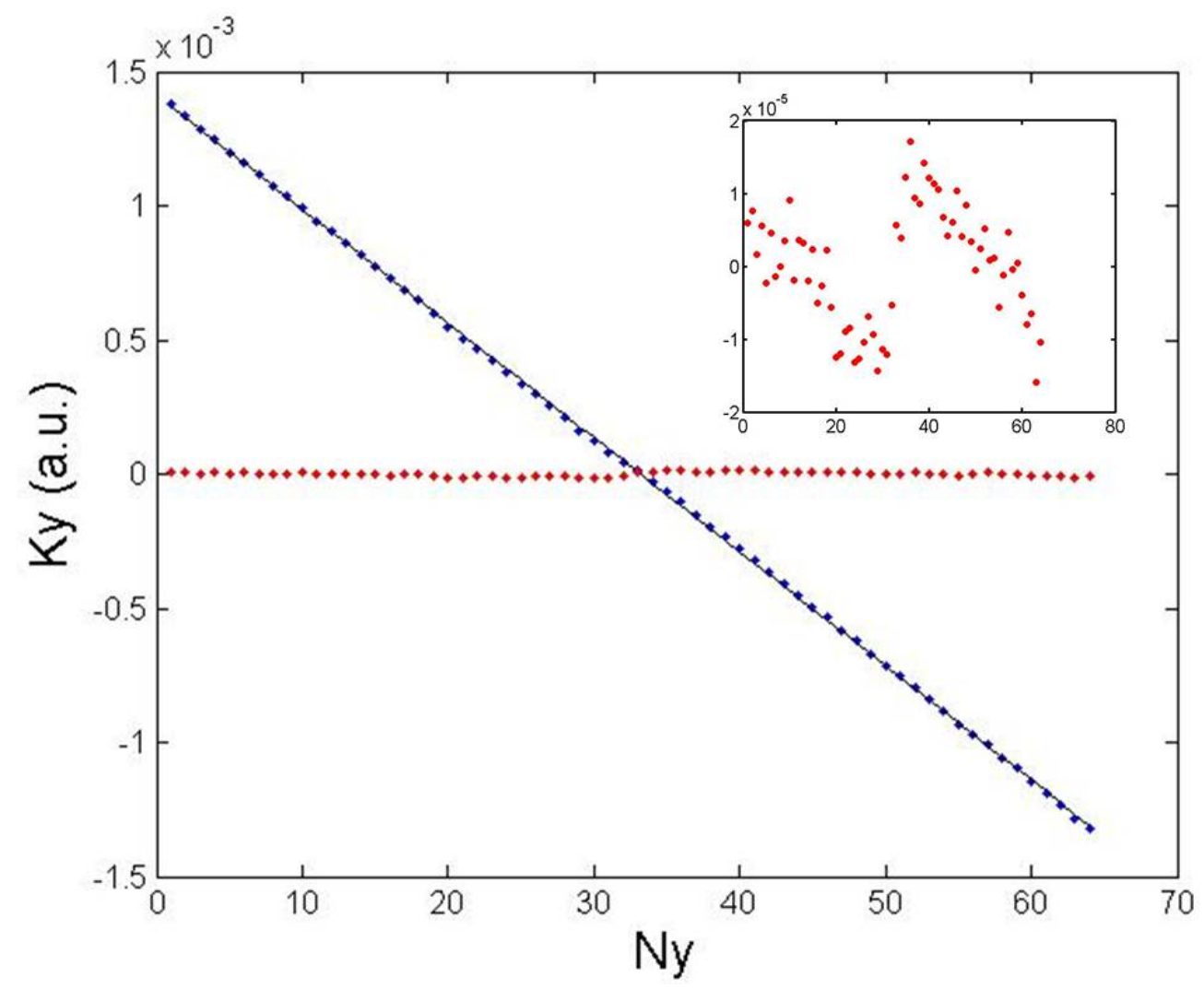

Figure 4.11: Measured $\mathbf{k}_{y}$ values with $\mathbf{k}_{x}=0$, its linear fit and residuals. The inset zooms in on the residuals and clearly shows that there is a particular pattern and not just random noise.

\subsubsection{Image Reconstruction}

In our gradient-echo acquisitions, $k$ space was sampled asymmetrically about $k_{x}=0$ in order to reduce diffusion attenuation of the NMR signal caused by the readout gradient. In addition, the data acquired during the first $6 \mathrm{~ms}$ of the readout gradient showed obvious irregularities for both ${ }^{3} \mathrm{He}$ and ${ }^{129} \mathrm{Xe}$ and were therefore discarded, which we believe was caused by gradients still approaching equilibrium values and/or the rining down of the receive chain after RF transmit. Thus the final $k$-space matrices were highly asymmetric in the $x$ direction. We also noticed a small DC offset in the 
raw k-space data. This offset was estimated for each channel (real and imaginary) by averaging the $\mathrm{k}$-space data measured at large $\mathrm{k}_{x}$ values, and the estimated offset was subtracted from all the raw k-space data. Magnitude images were then reconstructed by two-dimensional Fourier transform. For the ${ }^{3} \mathrm{He}$ phantom, we only reconstructed a single image. While for the Xe phantom, multiple averages were combined by first reconstructing each of the 9 image acquisitions separately and then coherently adding the complex pixel values.

In the fully phase-encoded acquisition, $k$ space was sampled symmetrically in both $x$ and $y$ directions. For each point in $k$ space, we had a long-lasting FID whose phase and amplitude contained the spatial frequency information. Since we also had a separate measurement of the resonant frequency for each $k$-space point, we corrected the FID phase by multiplying each ADC sample by the factor $e^{-i 2 \pi \Delta f t}$, where $\Delta f$ was the difference between the measured resonant frequency and the nominal Larmor frequency due to $B_{0}$ field drift (Fig. 4.9). We then Fourier-transformed each phase-corrected FID signal, yielding a frequency spectrum for each $k$-space point. Because of the phase correction, the desired spectral peak occurred at the same location for each FID. A narrow window centered on this location was chosen (width $\sim 0.5 \mathrm{~Hz}$ ) and the sum of the complex spectral values within this window was taken as the measured $k$-space value. A small DC offset was taken off using the same method as for the gradient echo images. We then corrected for gradient infidelity using $k$-space gridding reconstruction. As mentioned in the previous section, the actual $k_{y}$ coordinates deviated from the prescribed trajectory, whereas the $k_{x}$ trajectory was much more faithful. Using the measured $k_{y}$ coordinates, and assuming a perfectly linear $k_{x}$ trajectory, the measured $\mathrm{k}$ space values were resampled onto a rectilinear matrix by convolution with a finite function (Kaiser-Bessel gridding kernel) [95]. The final image was then reconstructed by taking the two-dimensional Fourier transform of the resampled $k$ space matrix and dividing by the Fourier transform of the gridding kernel.

\subsection{Results and Discussion}

\subsubsection{MR Images}

Images acquired using the gradient-echo pulse sequences are presented in Fig. 4.12 , Fig. $4.12(\mathrm{a})$ shows the image of the ${ }^{3} \mathrm{He}$ phantom. The in-plane spatial resolution is 1 


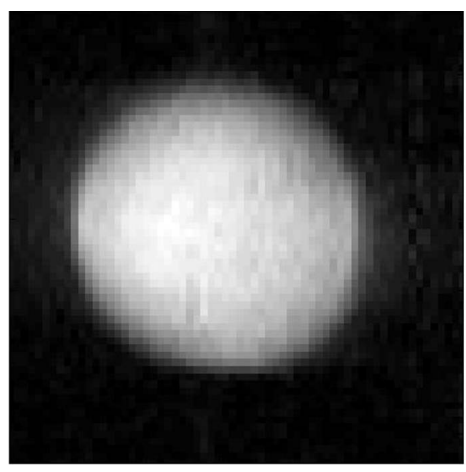

(a) Image of the ${ }^{3} \mathrm{He}$ phantom.

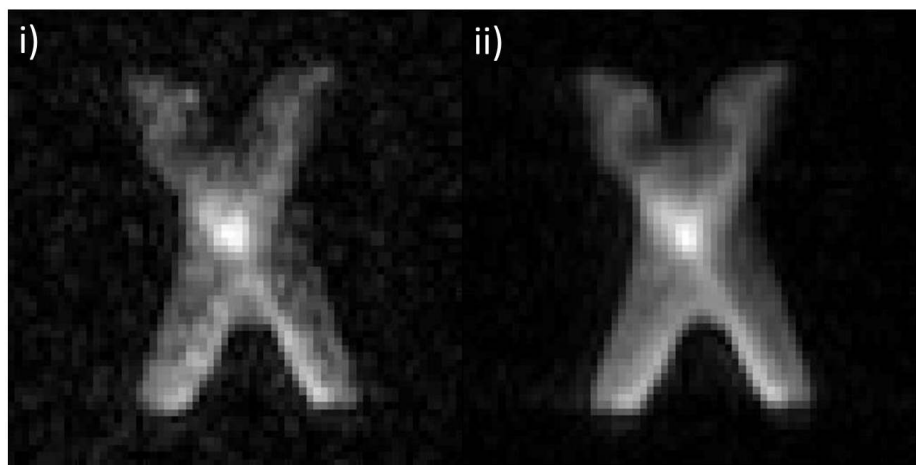

(b) Image of the ${ }^{129}$ Xe phantom, with 1 and 9 averages.

Figure 4.12: Images acquired by gradient echo acquisitions. The spatial resolution is interpolated from $1 \mathrm{~mm}$ to $0.5 \mathrm{~mm}$ for display here.

$\mathrm{mm}$. The phantom pull-off is not visible in the image since the amount of gas there was small and it was out of the sensitive range of the detection coils (Fig. 4.5(a). Images of the Xe phantom are shown in Fig. 4.12(b). Fig. 4.12(b)(i) shows the first of the nine identically acquired images and Fig. 4.12(b)(ii) shows the coherent average of all nine acquisitions. The basic structure of the X-shaped phantom is clear in both images, including the central bright spot at the location of the cell pull-off. We note, however, there appears to be some spatial distortion in the upper half of the phantom and artifactual signal enhancement along several of the edges.

Although the total time for each ${ }^{129} \mathrm{Xe}$ gradient-echo image acquisition was about half an hour, we emphasize that the vast majority of this time was spent waiting for the ${ }^{129} \mathrm{Xe}$ to re-polarize following each $90^{\circ} \mathrm{RF}$ pulse. That is, the long acquisition time was necessitated by the low ${ }^{129}$ Xe polarization achievable in our sealed cell rather than any fundamental limitations of low-field operation. With $50 \%$-polarized ${ }^{129} \mathrm{Xe}$ at atmospheric pressure, similar image SNR could be obtained in less than 4 seconds with the same pulse sequence by using the minimum possible repetition time $\left(T_{R} \sim 100 \mathrm{~ms}\right)$ and optimum constant flip angle $\left(\sim 14^{\circ}\right)$.

Images acquired using the fully phase-encoded pulse sequence are presented in Fig. 4.13. The first image (Fig. 4.13a) was reconstructed directly from the raw FID data without applying any corrections; the second image (Fig. $4.13 \mathrm{~b}$ ) was reconstructed after correcting for the measured $B_{0}$ field drift; and the third image (Fig. 4.13;) was corrected for both $B_{0}$ and $k$-space trajectory errors. There is a noticeable background 
"haze" surrounding the phantom in (a) that is greatly suppressed by the $B_{0}$ corrections applied in (b) and (c), and there are "double-edge" artifacts along the $y$ direction in (a) and (b) that are almost completely eliminated by the gridding reconstruction used in (c).

The nominal spatial resolution was $1 \mathrm{~mm}$ for our gradient-echo ${ }^{129} \mathrm{Xe}$ image and $0.5 \mathrm{~mm}$ for the fully phase-encoded image. It is worth noting that although our maximum gradient strength (less than $4 \mathrm{mT} / \mathrm{m}$ ) was much lower than that of commercial high-field scanners, the long $T_{2}^{*}$ at very low fields allowed us to achieve sub-millimeter spatial resolution by applying the gradients for a much longer time compared with the high field case to obtain the required gradient moment. For example, in the fully phase-encoded acquisition, the maximum gradient needed for $0.5 \mathrm{~mm}$ resolution was achieved by turning on the gradients at $\sim 3.3 \mathrm{mT} / \mathrm{m}$ for $25 \mathrm{~ms}$.

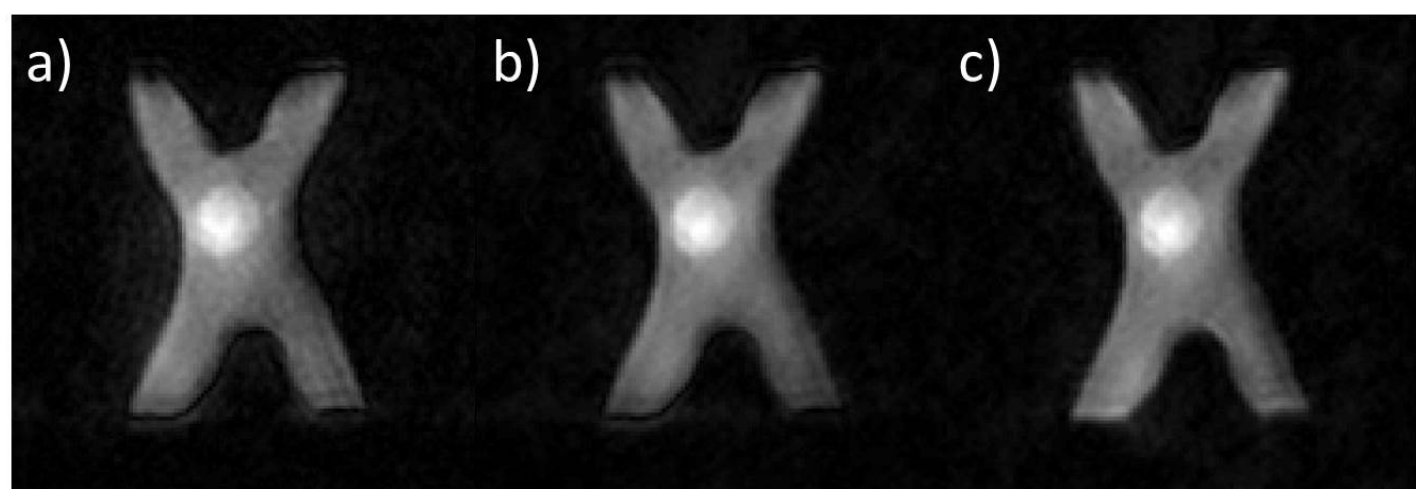

Figure 4.13: Fully phase encoded images with different levels of postprocessing. (a): image reconstructed from original data. (b): the holding field drift is corrected for. (c): In addition to (b), gridding reconstruction is also applied to account for the $k_{y}$ nonlinearity. The central bright area in these images are the phantom pull-off, as shown in Fig. 4.5(b)(a). The pixel size was interpolated from $0.5 \mathrm{~mm}$ to $0.25 \mathrm{~mm}$ for display here.

\subsubsection{Advantages of a Fully Phase-Encoded Image Acquisition}

One of the most attractive and potentially useful features of low-field operation is the long $T_{2}^{*}$ achievable in a well-shimmed holding field (Fig.4.6). Several groups have taken advantage of this feature to enable single-shot in-vivo imaging of ${ }^{3} \mathrm{He}$ at moderately low fields using long spin-echo trains [74, 76, 78]. However, since the imaged medium 
is a gas, diffusion sets a limit on how long we can acquire data in the presence of a frequency-encoding gradient. Movement of the noble-gas nuclei during the gradient will not only compromise the precision of the spatial encoding, but more importantly will attenuate the magnitude of the NMR signal.

We estimated that at the temperature and pressure of our phantom during imaging, the ${ }^{129} \mathrm{Xe}$ diffusivity was $0.023 \mathrm{~cm}^{2} / \mathrm{s}$, and the one-dimensional diffusion length in $70 \mathrm{~ms}$ was $\sim 0.6 \mathrm{~mm}$, which was of the same order of the pixel resolution of our gradient-echo acquisition. However as mentioned in Section 4.4.1, the $b$ value of a symmetric frequency-encoding gradient waveform would have been $33 \mathrm{~s} / \mathrm{cm}^{2}$, leading to diffusion-induced signal attenuation greater than $50 \%$ at the central $k$-space sample. This consideration led us to use a highly asymmetric readout to achieve the desired level of spatial resolution. The main point here is that xenon self-diffusion prevented us from taking full advantage of the long $T_{2}^{*}$ at very low field by using a frequency-encoded image acquisition.

On the other hand, a fully phase-encoded image acquisition is more immune to the effects of gas diffusion since the spatial-encoding gradients are only applied for a short time. Once the phase-encoding gradient pulses finish, the relative phase of the spins are determined. As a result, diffusion becomes largely irrelevant and we can acquire the NMR signal for as long as possible without suffering further diffusioninduced signal attenuation. Thus a fully phase-encoded acquisition provides a way to improve SNR by making use of the long $\mathrm{T}_{2}^{*}$ at low field. Furthermore, a fully phaseencoded image acquisition is a variation of Constant Time Imaging (CTI), which is naturally immune to distortions due to $\mathrm{B}_{0}$ field inhomogeneity, magnetic susceptibility variation and chemical shift [96]. Although high SNR resulting from our very long signal acquisition time is partially responsible for the dramatically better image quality of the fully phase-encoded acquisition in Fig. 4.13 , it is also clear from the gradientecho images in Fig. 4.12(b) that no amount of signal averaging using this gradient-echo acquisition would make these images look as good as our fully phase-encoded image, since the main imperfections associated with these images are unrelated to SNR.

Our fully phase encoded pulse sequence is essentially identical to a chemical shift imaging (CSI) acquisition. CSI pulse sequences are typically used to acquire spatially resolved NMR frequency spectra [97]. Although in our phantom there was only one frequency compartment (gas phase ${ }^{129} \mathrm{Xe}$ ), it is natural to consider whether it would be 
possible to perform simultaneous gas phase and dissolved phase in-vivo ${ }^{129} \mathrm{Xe}$ imaging at low field using this technique. The chemical shift between the two phases of ${ }^{129} \mathrm{Xe}$ is about $200 \mathrm{ppm}$ [98. Therefore at $2 \mathrm{mT}$ the frequency difference is about $5 \mathrm{~Hz}$. If the low-field $T_{2}^{*}$ is long enough to allow a frequency resolution better than $5 \mathrm{~Hz}$, then it might be possible to separate these two frequency compartments.

The $T_{2}^{*}$ of inhaled hyperpolarized gases will be much shorter than in our phantoms due to magnetic susceptibility variations in the lung. Wong et al. reported $T_{2}^{*}$ values a little over $100 \mathrm{~ms}$ for laser-polarized ${ }^{3} \mathrm{He}$ at $2 \mathrm{mT}$ in excised rat lungs [69]. Scaling by the gyromagnetic ratio, we estimate that in-vivo gas phase ${ }^{129} \mathrm{Xe} T_{2}^{*}$ could be $\sim 300 \mathrm{~ms}$ at low fields. This implies a spectral linewidth of $1 /\left(\pi T_{2}^{*}\right) \sim 1 \mathrm{~Hz}$ for the gas-phase peak, which is substantially smaller than the $5 \mathrm{~Hz}$ difference between peaks. However, the dissolved-phase $T_{2}^{*}$ is likely to be shorter than in the gas phase. Mugler et al. measured the $T_{2}^{*}$ of gas-phase and dissolved-phase ${ }^{129} \mathrm{Xe}$ to be $43 \mathrm{~ms}$ and $2 \mathrm{~ms}$, respectively, at $1.5 \mathrm{~T}$ in human lungs [99]. The corresponding values have not yet been reported at low fields. If the dissolved phase $T_{2}^{*}$ is much shorter than $300 \mathrm{~ms}$, separating the two compartments will be quite challenging using low-field CSI.

\subsubsection{In Vivo MRI at Low Fields}

An important extension of the work presented here would be low-field imaging of inhaled

${ }^{129} \mathrm{Xe}$ in animal or human subjects. In-vivo ${ }^{3} \mathrm{He}$ MRI has been performed in human lungs at field strengths around $6 \mathrm{mT}$ using homebuilt systems [79, 80, 100]. Our lowfield system, which has very good field uniformity over a $5 \times 5 \times 5 \mathrm{~cm}$ volume, would be quite suitable for imaging rodent lungs. In addition, the open geometry of the Helmholtz coils allows easy access to the imaged subject, which would be useful for accommodating the necessary ventilation and gas-delivery systems for in-vivo imaging.

On the pulse sequence side, there are several important differences between in-vivo lung MRI and in-vitro MRI that must be considered. As discussed in the previous section, $T_{2}^{*}$ will be much shorter in vivo. A reasonable estimate for $T_{2}^{*}$ of ${ }^{129} \mathrm{Xe}$ in rat lungs is $\sim 300 \mathrm{~ms}$ at $2 \mathrm{mT}$, which is an order of magnitude smaller than in our phantom. Second, gas diffusion is generally slower in lung airspaces due to the restrictions imposed by the alveolar microstructure. The apparent diffusion coefficients of ${ }^{3} \mathrm{He}$ and ${ }^{129} \mathrm{Xe}$ were measured to be $\sim 0.2 \mathrm{~cm}^{2} / \mathrm{s}$ [101] and $0.027 \mathrm{~cm}^{2} / \mathrm{s}$ in rat lungs [102 respectively, which are much smaller than the free diffusivity of ${ }^{3} \mathrm{He}$ or ${ }^{129} \mathrm{Xe}$ measured with air at 
atmospheric pressure $\left(0.86 \mathrm{~cm}^{2} / \mathrm{s}\right.$ and $\left.0.1 \mathrm{~cm}^{2} / \mathrm{s}\right)$. Our phantoms have $\sim 2.8$ atm of either ${ }^{3} \mathrm{He}$ or ${ }^{129} \mathrm{Xe}$. Under the actual imaging conditions, the diffusion coefficient of ${ }^{3} \mathrm{He}$ is about $0.87 \mathrm{~cm}^{2} / \mathrm{s}$, and that of Xe is about $0.023 \mathrm{~cm}^{2} / \mathrm{s}$.

For ${ }^{3} \mathrm{He}$ in-vivo MRI, our gradient echo pulse sequence can be applied with minimal modifications. Although we used a repetition time (TR) of $2 \mathrm{~s}$, we note that in principle the choice of TR is not important as long as it is much shorter than the ${ }^{3} \mathrm{He}$ SEOP time constant, which in our case is several hours. In practice, however, TR should be short enough for in-vivo lung MRI so that all data can be taken within a single breath hold. In our case, TR can be set shorter than $35 \mathrm{~ms}$, thus all 32 lines can be acquired in about $1 \mathrm{~s}$. The ${ }^{3} \mathrm{He}$ density will be lower in rat lungs than in our high pressure phantom by at least two thirds, but the smaller diffusion attenuation will partly compensate the signal loss.

In-vivo Xe MRI is very different. Unlike in the phantom, we cannot repolarize Xe inside rat lungs. Also, the animal must breathe during the long imaging process. Therefore a reservoir of hyperpolarized Xe and a rodent ventilation machine need to be involved. Hyperpolarized-gas images of mechanically ventilated rodents are often obtained by segmenting the acquisition so that it can be performed over many short breath holds. Nouls et.al. reported a ventilation machine with a cycle of $600 \mathrm{~ms}$, consisting of a $150 \mathrm{~ms}$ inhalation of an air/hyperpolarized gas mixture, a $300 \mathrm{~ms}$ breath hold, and a $150 \mathrm{~ms}$ exhalation [103]. The minimum possible TR of the gradient-echo pulse sequence demonstrated here is about $100 \mathrm{~ms}$, so up to three $k$-space lines could be acquired per breath hold, and a 32-line image could be acquired in less than 10 seconds. As mentioned earlier, xenon polarization above $50 \%$ can be obtained from the type of polarizers used for medical imaging, which is almost ten times larger than we can achieve in our high-pressure phantom. Assuming three-fold lower xenon density in vivo, and further simply assuming that the signal in each inhaled bolus is split evenly among the three line acquisitions, then with a ready supply of highly polarized ${ }^{129} \mathrm{Xe}$ gas we can expect to achieve roughly similar SNR in-vivo as we achieved in our singleaverage gradient-echo image shown in Fig. 4.12(b)(i). If we instead acquire only one $k$-space line per bolus, naively our signal will be three times larger and we can expect to achieve SNR similar to that of the 9-average phantom image in Fig. 4.12(b)(ii).

Since the expected Xe $T_{2}^{*}$ in vivo is similar to a typical breath-hold duration for rodent imaging, we could make optimum use of a fully phase-encoded acquisition with 
FID readout, by acquiring one $k$-space point per breath hold. Although the particular acquisition demonstrated here, which sampled a full $64 \times 64$ matrix, would be prohibitively long for general use in vivo, a $32 \times 32$ matrix could be sampled in 10 minutes. Furthermore, since a randomly undersampled point-by-point acquisition naturally lends itself to compressed sensing reconstruction, the required number of $k$-space points (and thus the total imaging time) could be significantly reduced, without sacrificing spatial resolution or imaging field-of-view, by incorporating such a strategy. 


\section{5}

\section{Polarized Nuclear Imaging (PNI) and Polarized Nuclear Detection (PND)}

In this chapter I'll describe a novel imaging modality called Polarized Nuclear Imaging (PNI) and a more general platform technique called Polarized Nuclear Detection (PND). These new platform techniques combine principles related to NMR with radiation detection. Basically, some radioactive nucleus shows a radiation emission asymmetry when polarized. We manipulate the polarized nuclear spins using NMR techniques as before, but detect the spin motion by gamma rays rather than radio-frequency electromagnetic waves.

PND detects MR properties of spins using radiation detection. PNI is actually an marriage of MRI and PND. This new technique keeps the great flexibility of MRI while also taking advantage of the high sensitivity of gamma detection, which might be quite suitable for some niche applications where it is difficult to introduce a large amount of contrast nuclei.

We also developed an imaging pulse sequence that is specially suited for PNI. The sequence, which we refer to as the Cates-Miller-Zheng or CMZ sequence, is a fully phase encoded pulse sequence and has several special features. First, it reads a DC signal instead of an oscillation signal, which we will describe more shortly. Second, the readout is most efficient along the holding field direction where there is no signal for conventional MRI. Third, the limit of sampling time is set by longitudinal relaxation instead 
of transverse relaxation. The transverse relaxation of nuclei with complicated spin structures can be quite fast, but we can still achieve a much longer than conventional readout time using the $\mathrm{CMZ}$ sequence.

\subsection{Radiation Asymmetry from Aligned Nucleus}

It has long been known that certain radioactive nuclei, when aligned, show a spatial anisotropy in radiation emission. The angular dependence of gamma emission from polarized nuclei can be described as the combination of a series of even Legendre polynomials $P_{k}(\cos \theta)$ [12, 104]:

$$
W(\theta)=1+A_{2} P_{2}(\cos \theta)+A_{4} P_{4}(\cos \theta)+\cdots
$$

where $\mathrm{W}$ is the differential probability of a photon being emitted at an angle $\theta$ from the polarization axis. The coefficients $\mathrm{A}_{k}$ can be expressed as:

$$
A_{k}=\rho_{k} F_{k}
$$

where $\rho_{k}$ is the orientation parameter and $\mathrm{F}_{k}$ depends on the spin sequence and multipolarity of the decay [105]. The orientation parameters can be calculated by [12]:

$$
\rho_{k}=(2 J+1)^{1 / 2} \sum_{m}(-1)^{J-m} \times C(J J k ; m-m 0) a_{m},
$$

where $\mathrm{C}$ is the Clebsch-Gordan coefficient, $\mathrm{J}$ is the spin of the nucleus, and $a_{m}$ is the normalized population of the $\mathrm{m}$ state.

The radioactive nucleus of special interest to us is ${ }^{131 \mathrm{~m}} \mathrm{Xe}$. The ${ }^{131 \mathrm{~m}} \mathrm{Xe}$ nucleus decays from the $\frac{11}{2}^{-}$metastable state to the $\frac{3}{2}^{+}$ground state by emitting a $164 \mathrm{keV}$ gamma ray. The $\mathrm{F}_{k}$ parameters are determined by the spin levels of of a certain decay. For the ${ }^{131 \mathrm{~m}} \mathrm{Xe} 164 \mathrm{keV}$ decay branch, the nonzero $\mathrm{F}_{k}$ parameters are $\mathrm{F}_{0}=1$, $\mathrm{F}_{2}=-0.8890, \mathrm{~F}_{4}=0.4434, \mathrm{~F}_{6}=0.0320$ and $\mathrm{F}_{8}=-0.2624$ [12]. Higher order $\mathrm{F}_{k}$ terms are zero. Thus Eq. 5.1 terminates at $\mathrm{k}=8$. Expanding the Legendre polynomials and simplifying the trigonometric expressions, Eq. 5.1 can be rewritten as:

$$
W(\theta)=a_{0}+a_{2} \cos (2 \theta)+a_{4} \cos (4 \theta)+a_{6} \cos (6 \theta)+a_{8} \cos (8 \theta),
$$

where $a_{k}$ are the coefficients of the Fourier components, and they depend on the polarization of the ${ }^{131 \mathrm{~m}}$ Xe nucleus. The $a_{k}$ parameters are plotted as a function of polarization in Fig. 5.1. When ${ }^{131 \mathrm{~m}}$ Xe nucleus is completely unpolarized, the only nonzero 
Fourier coefficient is $a_{0}$, which indicates an isotropic gamma distribution as one would expect. As the polarization increases, the magnitude of the parameter $a_{2}$ becomes bigger, which means the gamma distribution will show some anisotropy. For polarization greater than $70 \%, a_{4}$ also starts to increase, which makes the angular distribution even more complicated. Luckily, $a_{6}$ and $a_{8}$ remain small for all polarizations and can usually be ignored.

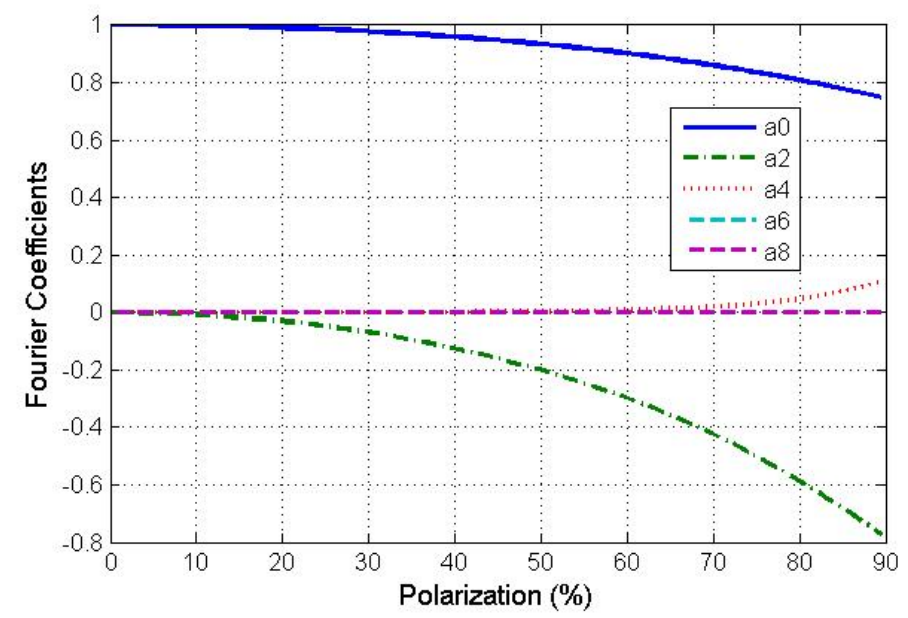

Figure 5.1: The Fourier coefficients of the ${ }^{131 \mathrm{~m}} \mathrm{Xe} 164 \mathrm{keV}$ gamma emissions as a function of polarization.

One special feature of the ${ }^{131 \mathrm{~m}} \mathrm{Xe} 164 \mathrm{keV}$ decay anisotropy is that when the radioactive xenon nucleus is $100 \%$ polarized, the gamma ray spatial asymmetry is quite high and no photons are emitted along the polarization direction, as shown in Fig. 5.2 .

\subsection{PND and PNI}

\subsubsection{Polarized Nuclear Detection (PND)}

In 1985, Calaprice et. al. aligned the nuclear spin of some radioactive noble gases using the technique of SEOP and studied their magnetic moment by measuring the gamma emission asymmetry [12]. Soon after that more experiments were conducted using the same technique [49, 106]. These experiments located NMR lines of various 


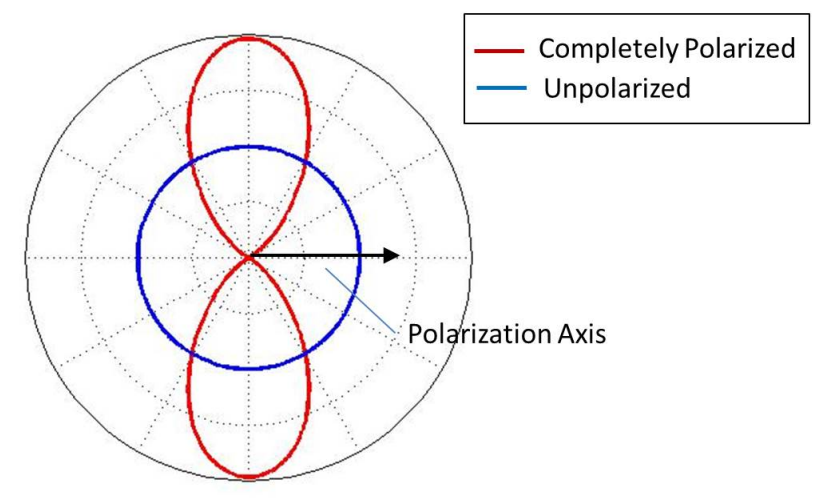

Figure 5.2: Angular distribution of ${ }^{131 \mathrm{~m}} \mathrm{Xe} 164 \mathrm{keV}$ gamma rays with $0 \%$ and $100 \%$ polarization.

radioactive nuclei by monitoring the change of photon count rates while disturbing the nuclear polarization by broadcasting an RF frequency. However, the count rates were measured when the nuclear spins reached some "equilibrium state", i.e. they didn't measure transient spin evolution in the magnetic field such as spin precession.

Actually, just like ${ }^{3} \mathrm{He}$ and ${ }^{129} \mathrm{Xe}$, the radioactive noble gas spins will precess along the external holding field once they are tipped away from the longitudinal direction. If a detector is placed in the transverse plane, the angle $\theta$ between the spin direction and the detector depends on both the polar angle between the spin and holding field, and the azimuthal angle that oscillates at the Larmor frequency $\mathrm{f}_{0}$. In a time period that is short compared with $\mathrm{T}_{1}$, the polar angle stays the same and the angle $\theta$ also oscillates at $\mathrm{f}_{0}$, which leads to a periodic change of the gamma ray count rate (Fig. 5.3). However, it is worth noting that the count rate doesn't oscillate at $\mathrm{f}_{0}$. From Eq. 5.4, it is clear that for ${ }^{131 \mathrm{~m}} \mathrm{Xe}$ there are components corresponding to different oscillating frequencies. The $\mathrm{a}_{0}$ term corresponds to a DC offset in the count rate, and the $\mathrm{a}_{2}$, $\mathrm{a}_{4}, \mathrm{a}_{6}$ and $\mathrm{a}_{8}$ terms correspond to count rates oscillating at $2 \mathrm{f}_{0}, 4 \mathrm{f}_{0}, 6 \mathrm{f}_{0}$ and $8 \mathrm{f}_{0}$. An important feature of PND is that although there is no count rate oscillation for spins completely out of phase in the transverse plane, the count rate still shows an anisotropy that depends on the polar angle made by the detector and the holding field.

Since spins of radioactive nuclei have to be polarized to show a radiation asymme- 


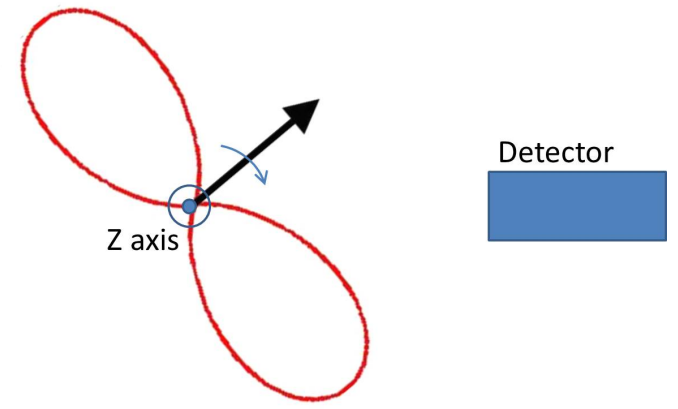

Figure 5.3: Count rate asymmetry from precessing ${ }^{131 \mathrm{~m}}$ Xe nuclei. The spins precess around a holding field that points out of the paper. The detector senses a change in count rate.

try, we call the technique of measuring spin behavior by radiation detection Polarized Nuclear Detection (PND).

\subsubsection{Basics of Polarized Nuclear Imaging (PNI)}

The technique of MRI is based on measuring the "k-space" (in spatial frequency domain) data by applying appropriate gradients to vary the phase of spins. The MRI signal is always detected by placing a coil near the sample, in which an emf will be induced by the change of magnetic flux. However, if the phantom contains polarized radioactive nuclei with an emission anisotropy, the spatial frequency signal can be collected by measuring the count rate rather than by E\&M induction. We call this new imaging technique, which combines spatial encoding and radiation detection, Polarized Nuclear Imaging (PNI).

Assume the spins are tipped to the transverse plane and then imaging gradients are applied. The phase of spins at position $\vec{r}$ evolves as $\Phi(t, \vec{r})=2 \pi\left(f_{0} t+\gamma \vec{G} \cdot \vec{r} t\right)$. The the count rate measured by a detector placed at the transverse plane is:

$$
\begin{aligned}
R(t) & =C \int \rho(\vec{r}) W(\Phi(t, \vec{r})) d \vec{r} \\
& =C \int \rho(\vec{r}) \sum_{m=0,2,4, \cdots} a_{m} \cos (m \Phi(t, \vec{r})) d \vec{r}
\end{aligned}
$$


Where $\mathrm{C}$ depends on the source activity, detector efficiency and the geometries. We ignored here the weak spatial dependence of C. For the special case of ${ }^{131 \mathrm{~m}}$ Xe with less than $70 \%$ polarization (which is the case in our experiment), the parameters $a_{4}, a_{6}$ and $a_{8}$ are very small and can be ignored (Fig. 5.1). With this assumption, Eq. 5.5 can be simplified to:

$$
\begin{aligned}
R(t) & =C \int \rho(\vec{r})\left[a_{0}+a_{2} \cos (2 \Phi(t, \vec{r}))\right] d \vec{r} \\
& =C \int \rho(\vec{r})\left[a_{0}+a_{2} \cos \left(2 \pi\left(2 f_{0} t+2 \gamma \vec{G} \cdot \vec{r} t\right)\right)\right] d \vec{r}
\end{aligned}
$$

The $a_{0}$ term corresponds to a DC offset in the count rate, which must be subtracted before further data analysis. The oscillating $a_{2}$ term has a carrier frequency of $2 \mathrm{f}_{0}$, which in this case equals twice the Larmor frequency. The spatial frequency information, which is contained in $2 \gamma \vec{G} \cdot \vec{r} t$, can be recovered by demodulation of the oscillating count rate. With the definition that $\vec{k}=2 \gamma \vec{G} t$ (note that there is an extra factor of 2 compared with the definition of $\mathrm{k}$ in conventional MRI), Eq. 5.6 becomes:

$$
R(\vec{k})=C \cdot \operatorname{Re}\left(\int \rho(\vec{r}) a_{2} e^{2 \pi i \vec{k} \cdot \vec{r}} d \vec{r}\right)
$$

The imaginary part of the $\mathrm{k}$ space data $R^{\prime}(\vec{k})$ can also be measured using a slightly different pulse sequence strategy, which will be discussed later. The spatial distribution of the spins $\rho(\vec{r})$ can be reconstructed by Fourier transforming $s(\vec{k})=R(\vec{k})+i R^{\prime}(\vec{k})$ as shown in Eq4.5.

PNI provides a new modality for imaging and it has several advantages over conventional nuclear imaging techniques and MRI:

- A conventional gamma camera has a collimator in front of a crystal/PMT array. The collimator is usually a thick sheet of lead with thousands of adjacent holes through it, which usually allows between $0.1 \%$ and $1 \%$ of incident photons to reach the crystals. Using PNI, an image can be produced using a single detector with no collimator, which has the potential of dramatically enhancing the sensitivity over conventional nuclear imaging.

- MRI detects the change of magnetic flux generated by protons in water molecules on a macroscopic level, while PNI is much more sensitive and can detect a single gamma ray. If a 32 by $32 \mathrm{k}$ space matrix is covered using PNI, and for each 
point on the matrix we acquired $10^{4}$ counts to achieve a statistical error less than $1 \%$ (including a DC offset from the $\mathrm{a}_{0}$ term), the total number of photons involved is only $10^{7}$. The actual number of radioactive nuclei needed can be several orders of magnitude bigger depending on the radiation anisotropy, the branch ratio of the useful decay, the efficiency of the detector, the geometries of the detector and the source, and the fraction of radioactive nuclei decayed during the imaging procedure. However, if we include another factor of $10^{4}$ to account for all these non-idealities, the amount of the radioactive gas needed is still only $\sim 4 \times 10^{-9} \mathrm{~atm} \cdot$ c.c. .

- PNI is naturally suited for operating at low field. The signal size of conventional proton MRI strongly depends on the holding field $\left(\propto B_{0}^{2}\right)$, because both the proton polarization and precession frequency are proportional to the $\mathrm{B}_{0}$ field. In hyperpolarized gas MRI, the noble gas polarization is determined by the polarization process, thus the signal size only depends on $\mathrm{B}_{0}$ linearly. In PNI, the radioactive noble gas is also hyperpolarized, thus its polarization is independent of the holding field. Furthermore, the signal size doesn't depend on the precession frequency, since the signal detection is based on the arrival of photons, not the rate of the magnetic flux change. Therefore, the signal size of PNI is completely independent of the $\mathrm{B}_{0}$ field. Actually it is even advantageous to operate at low field, since the slower precession rate allows a more accurate measurement of the spin precession given a certain temporal resolution of the signal processing electronics.

\subsubsection{PNI with a CMZ Pulse Sequence}

In principle, a lot of conventional MRI pulse sequences can be used for PNI. We first considered using the fully phase encoded pulse sequence as described in Chapter 4 (Fig. 4.8). However, as will be discussed later, ${ }^{131 \mathrm{~m}} \mathrm{Xe}$ has a rather fast transverse decay rate $\left(T_{2} \sim 150 \mathrm{~ms}\right.$, as shown in Fig. 5.16) due to quadrupole and higher order interactions, which makes it impossible to take data for long even in a very uniform holding field. The short data taking time means a small number of counts and therefore big statistical noise. To solve this problem, we developed a new imaging pulse sequence that we call the "Cates-Miller-Zheng" (CMZ) sequence, which is specially suited for 
PNI. The CMZ pulse sequence is also fully phase encoded, and the spatial encoding is performed by applying appropriate gradients after the spins are flipped to the transverse plane. However, we then apply another $90^{\circ} \mathrm{RF}$ pulse to flip the spins again before data taking(Fig. 5.4). ${ }^{131 \mathrm{~m}} \mathrm{Xe}$ is under continuous SEOP during an imaging procedure, and we take the next point in $\mathrm{k}$ space after it is repolarized.

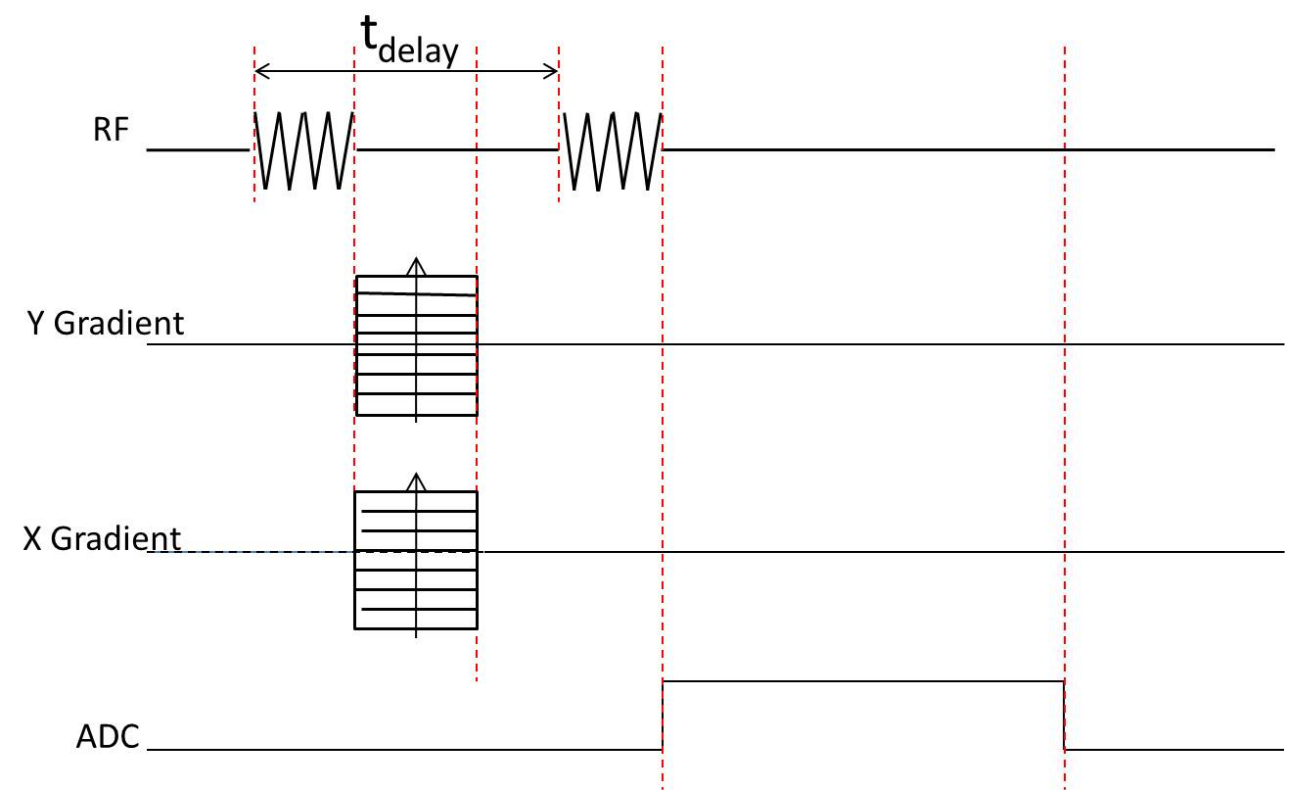

Figure 5.4: The CMZ pulse sequence. Counts of photons are acquired after the second RF pulse. Proper delays between the first and second RF pulse need to be chosen, as discussed later in this section.

I'll explain the CMZ pulse sequence in detail by tracking the classical motion of spins, as shown in Fig. 5.5. $\mathrm{x}-\mathrm{y}-\mathrm{z}$ is the lab frame and the holding field points along the $\mathrm{z}$ direction. The oscillating $\mathrm{RF}$ field is applied along the $\mathrm{x}$ direction. After the radioactive xenon is polarized, we broadcast a RF pulse to tip the spins to the transverse plane (along y axis for an instantaneous RF pulse). In the absence of gradients, the spins precess along $\mathrm{z}$ axis and the angle made by the spins and the lab frame y axis increases by $\Phi_{0}=f_{0} t$, where $f_{0}$ is the Larmor frequency and $\mathrm{t}$ is the time since the application of RF pulse. Now let's consider the spin motion with gradients applied immediately after the spins are tipped to the transverse plane. The effect of the gradients is that spins at position $\vec{r}$ feels an additional field that is well approximated by $\vec{G} \cdot \vec{r}$. Thus there is an extra precession angle $\Phi_{1}$. At some time $t_{0}, \Phi_{0}$ is $90^{\circ}$ and $\Phi_{1}$ can be calculated by 


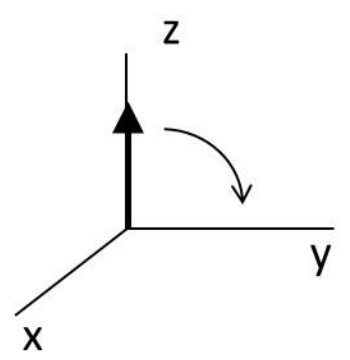

(a)

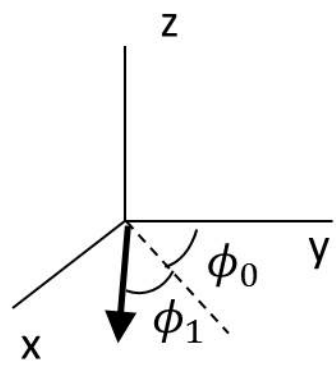

(d)

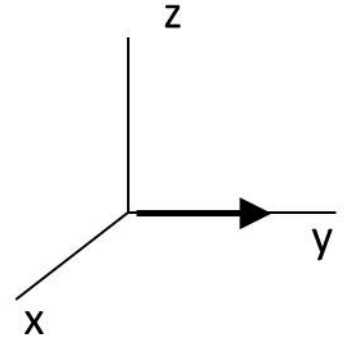

(b)



(e)

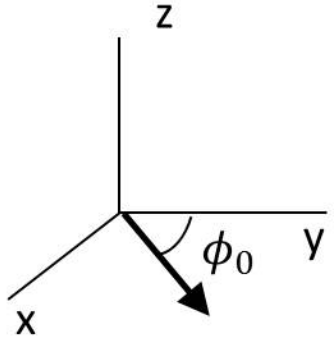

(c)

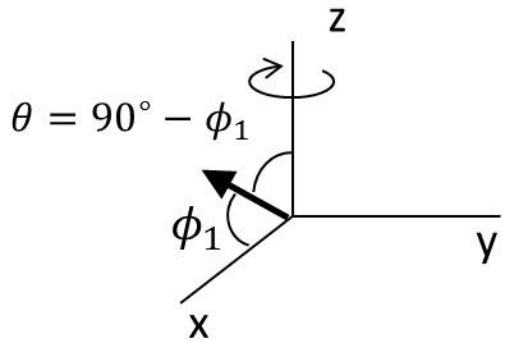

(f)

Figure 5.5: Spin motion during a CMZ sequence. $B_{0}$ is along the $z$ axis. (a) and (b) Apply a RF pulse to flip the spins to the transverse plane. (c) The angle made by the spin direction and y axis $\left(\Phi_{0}\right)$ increases with time. (d) With imaging gradients, there is an additional angle $\Phi_{1}$. (e) Apply a second $90^{\circ} \mathrm{RF}$ to tip spins on to the x-z plane. (f) The angle between the spins and the $\mathrm{z}$ axis depends on $\Phi_{1}$ and doesn't change with precession.

$2 \pi \gamma \vec{G} \cdot \vec{r} t_{0}$. A second $90^{\circ} \mathrm{RF}$ pulse is applied at this moment along x direction and the spins are flipped to the $\mathrm{x}-\mathrm{z}$ plane. As shown in Fig. 5.5 (f), the angle made by the $\mathrm{z}$ axis and the spins is $\theta=90^{\circ}-\Phi_{1}$, which won't change as the spin precesses along the $\mathrm{z}$ axis in a time much shorter than the spin-relaxation time constant.

Let's consider the count rate of a detector placed along the $\mathrm{z}$ axis after the second $90^{\circ}$ pulse from a continuous spin distribution. Using the gamma emission anisotropy defined in Eq. 5.4, we have:

$$
\begin{aligned}
R(t) & =C \int \rho(\vec{r}) W(\theta) d \vec{r} \\
& =C \int \rho(\vec{r})\left(a_{0}+a_{2} \cos (2 \theta)+a_{4} \cos (4 \theta)+a_{6} \cos (6 \theta)+a_{8} \cos (8 \theta)\right) d \vec{r}
\end{aligned}
$$




$$
\begin{aligned}
= & C \int \rho(\vec{r})\left(a_{0}-a_{2} \cos \left(2 \Phi_{1}\right)+a_{4} \cos \left(4 \Phi_{1}\right)-a_{6} \cos \left(6 \Phi_{1}\right)+a_{8} \cos \left(8 \Phi_{1}\right)\right) d \vec{r} \\
= & C \int \rho(\vec{r})\left(a_{0}-a_{2} \cos (2 \pi(\vec{k} \cdot \vec{r}))+a_{4} \cos (2 \pi(2 \vec{k} \cdot \vec{r}))\right. \\
& -a_{6} \cos (2 \pi(3 \vec{k} \cdot \vec{r}))+a_{8} \cos (2 \pi(4 \vec{k} \cdot \vec{r})) d \vec{r}
\end{aligned}
$$

where again we ignored the weak position dependence of the parameter C. $\vec{k} \equiv 2 \gamma \vec{G} t_{0}$ is the spatial frequency vector. Note the extra factor of 2 in the definition of $\vec{k}$ here.

If we apply the second $90^{\circ}$ pulse $1 / 8$ of a precession period later, $\Phi_{0}=135^{\circ}$ and $\theta^{\prime}=90^{\circ}-\left(\Phi_{1}+45^{\circ}\right)=45^{\circ}-\Phi_{1}$. Here we assume that the short extra delay time doesn't result in a change in $\Phi_{1}$, which is a good assumption in our case. Alternatively, the new $\theta^{\prime}$ can be achieved by applying $B_{1}$ with a $45^{\circ}$ different starting phase. The corresponding longitudinal count rate turns out to be:

$$
\begin{aligned}
R^{\prime}= & C \int \rho(\vec{r}) W\left(\theta^{\prime}\right) d \vec{r} \\
= & C \int \rho(\vec{r})\left[a_{0}+a_{2} \sin \left(2 \Phi_{1}\right)-a_{4} \cos \left(4 \Phi_{1}\right)-a_{6} \sin \left(6 \Phi_{1}\right)+a_{8} \cos \left(8 \Phi_{1}\right)\right] d \vec{r} \\
= & C \int \rho(\vec{r})\left[a_{0}+a_{2} \sin (2 \pi(\vec{k} \cdot \vec{r}))-a_{4} \cos (2 \pi(2 \vec{k} \cdot \vec{r}))\right. \\
& \left.-a_{6} \sin (2 \pi(3 \vec{k} \cdot \vec{r}))+a_{8} \cos (2 \pi(4 \vec{k} \cdot \vec{r}))\right] d \vec{r}
\end{aligned}
$$

Constructing a complex number $\mathrm{s}=\mathrm{R}+\mathrm{iR}$ ', we have

$$
\begin{aligned}
s(k)= & C \int \rho(\vec{r})\left\{a_{0}(1+i)-a_{2}[\cos (2 \pi(\vec{k} \cdot \vec{r}))-i \sin (2 \pi(\vec{k} \cdot \vec{r}))]\right. \\
& +a_{4}(1-i) \cos (2 \pi(2 \vec{k} \cdot \vec{r}))-a_{6}[\cos (2 \pi(3 \vec{k} \cdot \vec{r}))+i \sin (2 \pi(3 \vec{k} \cdot \vec{r}))] \\
& \left.+a_{8}(1+i) \cos (2 \pi(4 \vec{k} \cdot \vec{r}))\right\} d \vec{r} \\
& =-C \int \rho(\vec{r})\left[a_{2} e^{-i 2 \pi(\vec{k} \cdot \vec{r})}+\cdots\right] d \vec{r} .
\end{aligned}
$$

All but the $\mathrm{a}_{2}$ term are ignored in Eq. 5.10 . It is now clear that $s(\vec{k})$ and $\rho(\vec{r})$ are a Fourier transform pair. Therefore if we acquire a matrix of $s(\vec{k})$ point by point using the CMZ pulse sequence, an image of the spin spatial distribution can be reconstructed.

Although in the derivation above we assumed $\Phi_{0}=90^{\circ}$ and $135^{\circ}$ respectively for the count rate $\mathrm{R}$ and $\mathrm{R}$ ', we emphasize here that $\Phi_{0}$ can be any angles as long as the two $\Phi_{0}$ differ by $45^{\circ}$.

In Eq. 5.10, we only kept the $a_{2}$ term and omitted other $\left(a_{0}, a_{4}, a_{6}\right.$ and $\left.a_{8}\right)$ terms. These terms actually cause artifacts in the reconstructed image. The $a_{0}$ term is the 
same for all points and results in a DC offset in k space. This offset needs to be taken off before performing Fourier transform, otherwise the center of image space will have an artificial peak. The $a_{4}, a_{6}$ and $a_{8}$ terms lead to artifacts of various types like images on different scales and quadrature ghosts, which cannot be cleaned up easily. In principle, more measurements can be taken with different $\Phi_{0}$ angles, and the count rate due to different orders of anisotropy can be extracted from their linear combinations, i.e. the $a_{2}, a_{4}, a_{6}$ and $a_{8}$ terms can be separated. While in practice the $a_{6}$ and $a_{8}$ terms of ${ }^{131 \mathrm{~m}} \mathrm{Xe}$ are always very small, and the $a_{4}$ term can also be ignored for polarizations below $70 \%$ (Fig. 5.1).
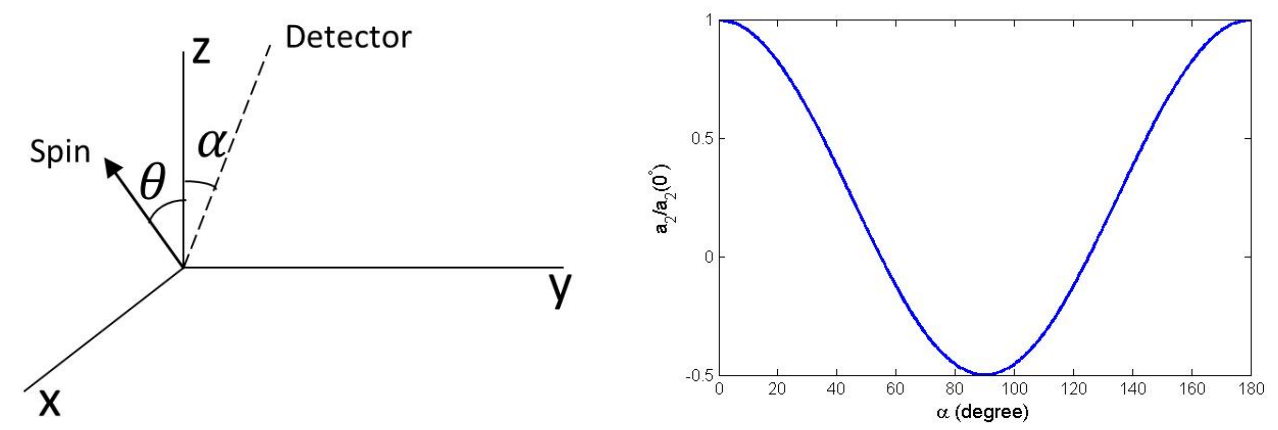

(a) Detector placed at a non-longitudinal di- (b) Angular dependence of the $a_{2}$ term on the derection. tector orientation $\alpha$.

Figure 5.6: ${ }^{131 \mathrm{~m}} \mathrm{Xe} 164 \mathrm{keV}$ gamma emission anisotropy as a function of the detector polar angle.

In the previous discussion we only considered measuring the count rate using the longitudinal detector. However, we emphasize here that the detector can be placed at almost any direction, and the longitudinal direction is just where it measures the biggest gamma asymmetry. For a detector placed at an angle $\alpha$ with respect to the longitudinal direction (Fig. 5.6(a) , the count rate has both a DC and a oscillation component due to spin precession. Although in theory both components can be used for imaging, the ${ }^{131 \mathrm{~m}}$ Xe nuclei dephase quickly ( $\left.150 \mathrm{~ms}\right)$, which limits the sampling time for the oscillating component and results in big statistical errors. On the other hand, the DC count rate only depends on the angle $\theta$ between the spins and the $\mathrm{z}$ direction and stays constant within a time short compared to the spin relaxation time 
constant. However, the anisotropy is diluted for detectors placed at non-longitudinal directions. For a detector placed at an angle $\alpha$ from the z direction, the coefficient of the $\cos (2 \theta)$ term can be approximated by $\frac{1+3 \cos (2 \alpha)}{4} a_{2}\left(0^{\circ}\right)$, ignoring contributions from higher order terms. The parameter $\mathrm{a}_{2}$ is plotted as a function of the detector angle $\alpha$ in Fig. 5.6(b), In general, we want $\mathrm{a}_{2}$ to be as big as possible for PNI. One special feature is that at the magic angle $\theta=54.7^{\circ}$ ( or $180^{\circ}-54.7^{\circ}$ ), the $\mathrm{DC}$ count rate doesn't depend on the spin direction polar angle $\theta$ and doesn't carry the spatial frequency information. Therefore this orientation should be avoided. At the transverse direction, we have $a_{2}\left(90^{\circ}\right)=-\frac{1}{2} a_{2}\left(0^{\circ}\right)$. Although the asymmetry is smaller and has a different sign, useful information can still be collected using transverse detectors.

We finish this section by comparing conventional MRI sequences with the CMZ sequence:

- Conventional pulse sequences measure oscillation signals while the CMZ sequence measures a DC signal whose magnitude encodes spatial information.

- There is a maximum signal in the transverse direction and no signal along the longitudinal direction for conventional pulse sequences. While for the CMZ pulse sequence, it is most efficient to take data in the longitudinal direction, though useful data can also be collected in the transverse plane.

- The readout time is limited by transverse relaxation for conventional pulse sequences. However, for a CMZ sequence this limit is set by longitudinal relaxation.

\subsection{PNI Setup}

\subsubsection{Overview of Our PNI System}

A large part of our setup for PNI is the same as the setup we used for low field hyperpolarized gas imaging (Section 4.3), such as the holding field coil, RF coil, gradient coils, gradient control system, SEOP lasers and optics, etc. However, the signal detection and processing systems are completely different. The detection coils are replaced by three gamma-ray detectors, one along the longitudinal direction and two in the transverse plane, as shown in Fig. 5.7. Signal processing electronics for voltages are replaced by those for gamma rays. 


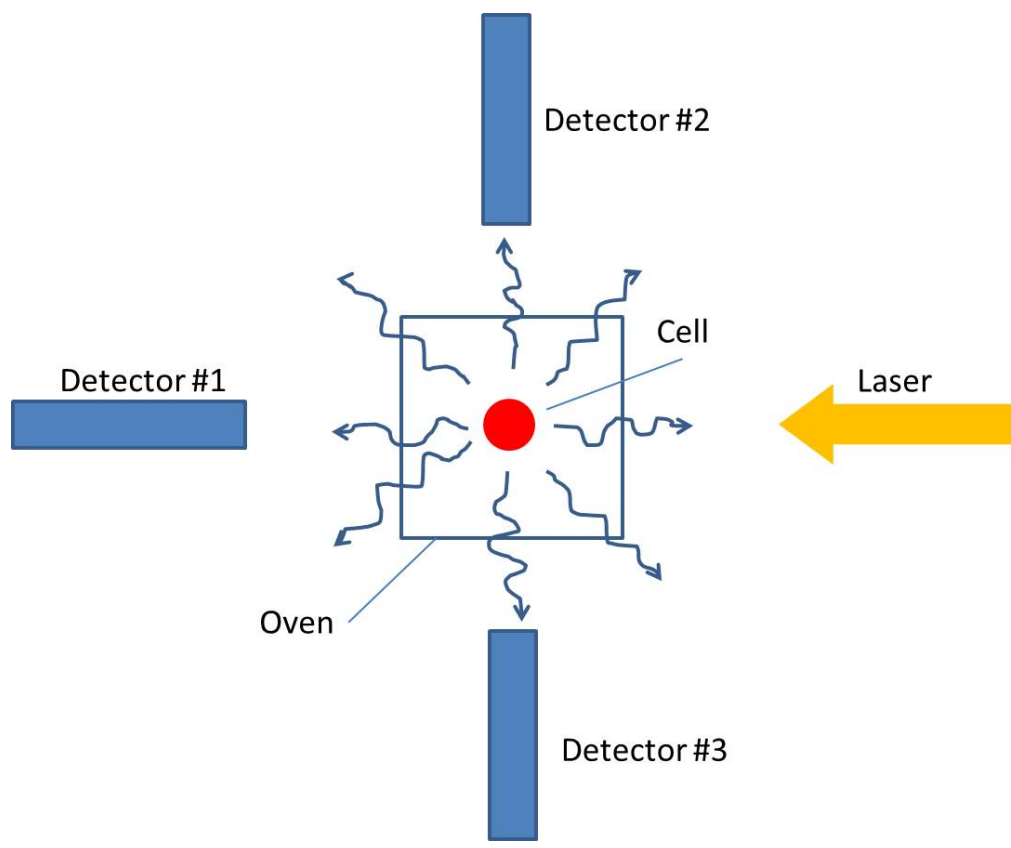

Figure 5.7: Detectors set along the longitudinal and transverse directions.

\subsubsection{Detectors and Electronics}

We used NaI crystals to detect the arrival of $164 \mathrm{keV}$ gamma rays from ${ }^{131 \mathrm{~m}}$ Xe decays. In lots of applications a Photo Multiplier Tube (PMT) is directly coupled to the back of the NaI crystal. PMTs are delicate vacuum tubes that can multiply the current produced by small amount of incident light by typically $10^{5}-10^{7}$ times. PMTs consist of a number of electrodes called dynodes. Each dynode is held at a more positive voltage than the previous one. Primary electrons are produced by the incoming photons hitting the photocathode by photoelectric effect. These electrons are then accelerated by the electric field and move towards the first dynode. When the accelerated electrons strike the first dynode, more free electrons are produced which move towards the second dynode. Obviously the motion of electrons in a PMT should not be disturbed by external electrical or magnetic fields, and that is why PMTs are often shielded.

In our experiment, the phantom is placed in a 6 to 20 Gauss holding magnetic field for SEOP and imaging, and the NaI crystal needs to be placed not too far away from the phantom to cover a reasonable fraction of the solid angle. We typically put the crystal about $13 \mathrm{~cm}$ away from the phantom. Considering the size of our holding Helmholtz coil (radius $66 \mathrm{~cm}$ ), the PMT will be well inside the Helmholtz coil if coupled directly to 


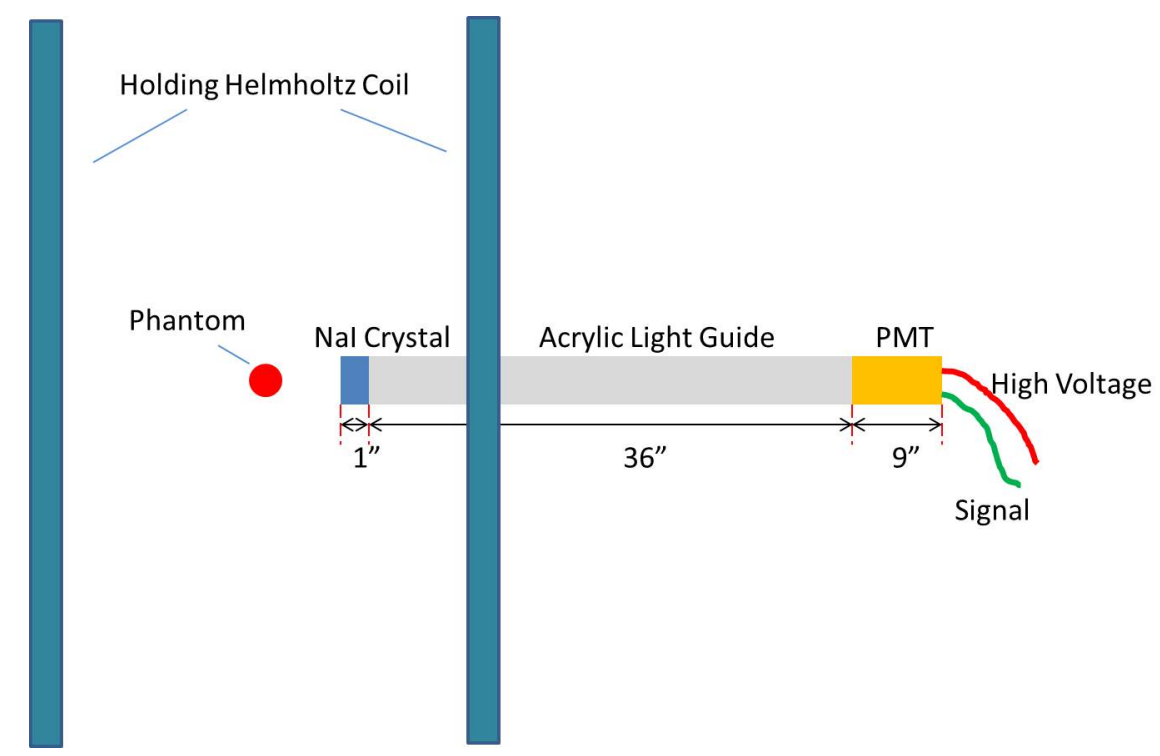

Figure 5.8: A NaI detector with a lightguide. The NaI crystal is close to the phantom, while the PMT is far away from it.

the NaI crystal. Not only will the PMT performance be compromised, the holding field that the phantom feels will also be disturbed, which is unfavorable for both SEOP and imaging. Therefore we added a long lightguide between the crystal and the PMT, which allowed the PMT to be far away from the isocenter of the system while keeping the NaI crystal close to the phantom, as shown in Fig. 5.8. The lightguide we used is simply an off-the-shelf polished acrylic rod. The disadvantage of having a long lightguide is that some photons will be lost while propagating in it, resulting in a worse energy resolution. However this is not a big problem in our experiment. First, the spectrum of ${ }^{131 \mathrm{~m}} \mathrm{Xe}$ decay is quite clean. There are only some low energy ( $\sim 30 \mathrm{keV}) \mathrm{X}$-rays besides the 164 $\mathrm{keV}$ gamma rays. Second, we are more interested in the timing information rather than knowing the energy accurately. Spectra of polarized and unpolarized ${ }^{131 \mathrm{~m}}$ Xe taken with our home-made detector are shown in Fig. 5.9.

The schematic of the signal processing and acquisition electronics is shown in Fig. 5.10. The output signal from our home-made detector was small and noisy. It was amplified and cleaned up by a shaping amplifier $(\tau=0.25 \mu \mathrm{s})$. The output of the shaping amplifier was sent to a single channel analyzer with the window set for the $164 \mathrm{keV}$ peak. When a $164 \mathrm{keV}$ photon arrives, a TTL pulse was generated, which was then sent to a gate/delay generator to trigger another TTL pulse with adjustable 


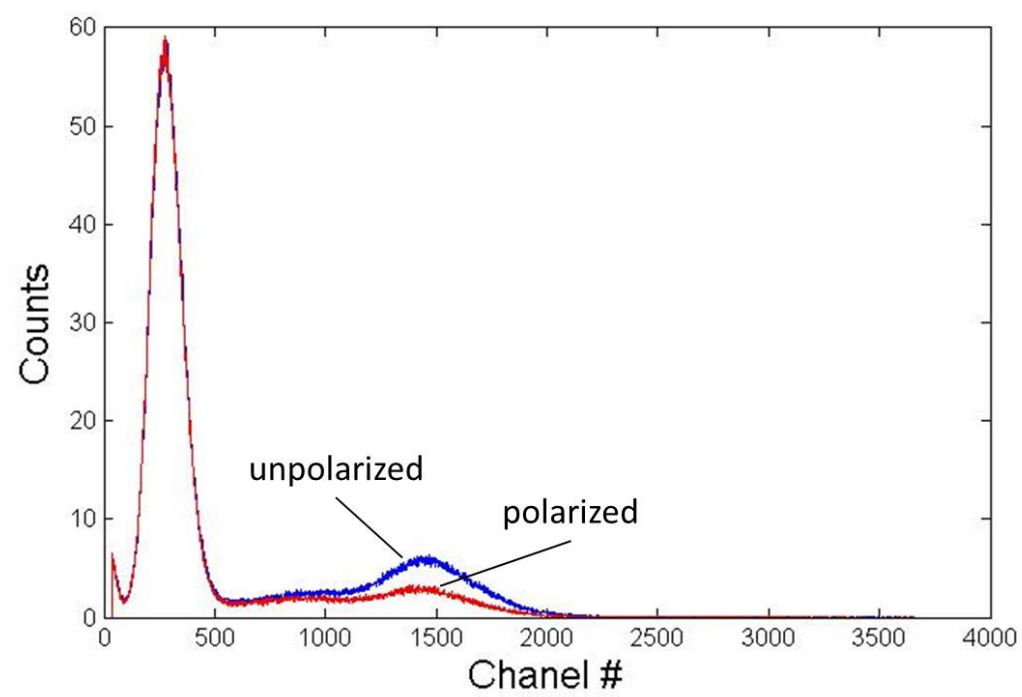

Figure 5.9: Polarized and unpolarized ${ }^{131 \mathrm{~m}}$ Xe decay spectra measured by the longitudinal detector. The peak on the left is from the $\sim 30 \mathrm{keV}$ X-rays. Peaks on the right are from the $164 \mathrm{keV}$ decays.

width for data acquisition. The typical pulse duration we use was $3.3 \mu \mathrm{s}$. A National Instrument data acquisition card recorded the output voltage of the gate/delay generator as a function of time. The data sampling frequency was set high enough that no pulses were missed. For example, we used a sampling frequency of $333 \mathrm{kHz}$ for $3.3 \mu \mathrm{s}$ wide pulses. Under our typical operating conditions, the photon arrival rate was very low compared with the data acquisition rate, and the voltage string was mostly zero. A new data string was then generated by picking out the rising slopes of the original voltage string, indicating arrivals of photons.

\subsubsection{Phantom Preparation}

We used ${ }^{131 \mathrm{~m}}$ Xe for our preliminary PNI studies because it has the following features:

1. It has a clean spectrum. As shown in Fig. 5.9, there are only two well separated peaks on a ${ }^{131 \mathrm{~m}} \mathrm{Xe}$ spectrum acquired by a NaI detector.

2. It is a noble gas and can be polarized by the technique of SEOP.

3. It has a decay branch $(164 \mathrm{keV})$ with a big gamma asymmetry (Fig 5.2$)$. 


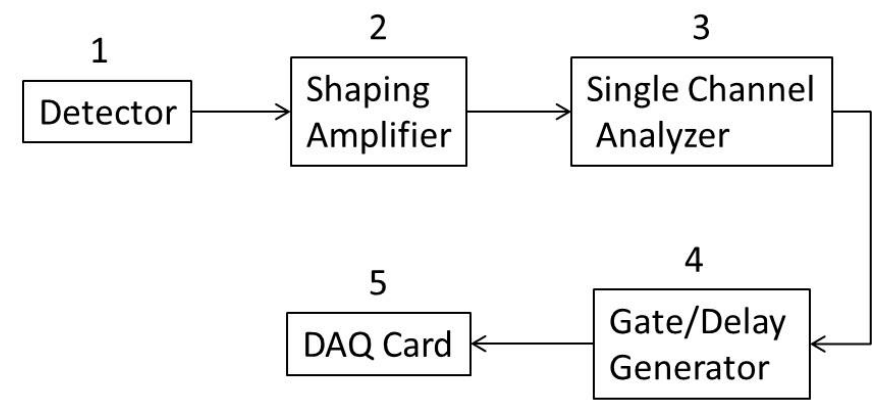

Figure 5.10: PNI signal processing schematic. The components are as follows: 1, Home-made NaI detector; 2, Canberra Spectroscopy Amplifier Model 2020; 3, Ortec Timing Single Channel Analyzer Model 420; 4, Philips Scientific Quad Gate/Delay Generator Model 794; 5, National Instrument Data Acquisition Card Model PCI-6259.

4. It is the decay product of the widely used nuclear medicine ${ }^{131} \mathrm{I}$ and is available to us.

The disadvantage of ${ }^{131 \mathrm{~m}} \mathrm{Xe}$, though, is that the branch ratio of the $164 \mathrm{keV}$ decay is $2 \%$, i.e. only a small fraction of the ${ }^{131 \mathrm{~m}} \mathrm{Xe}$ decays generate $164 \mathrm{keV}$ photons.

The typical dose of ${ }^{131 \mathrm{~m}} \mathrm{Xe}$ that we work with is between $500 \mu \mathrm{Ci}$ and $1 \mathrm{mCi}$, which is a very small amount. Our supply of ${ }^{131 \mathrm{~m}}$ Xe comes in a gas mixture $(\sim 10$ c.c. total volume) that is "dirty" (with water vapor and oxygen) for SEOP. Therefore we need to clean it up before filling a phantom.

The "dirty" gases were removed by sending the gas mixture into a "temporary" cell pre-filled with Rb, as shown in Fig. 5.11. The "temporary" cell (Cell 1, 1.25" I.D.) was a valved cell that was connected to the plumbing system by a removable chemthread connection. The whole system was first flushed with high purity $\mathrm{N}_{2}$ for several times with both valves open, and then left with one atmosphere of $\mathrm{N}_{2}$ in a state that the ballast bag was fully collapsed but not under vacuum. The valves V1 and V2 were then closed. The gas mixture was injected into the plumbing system by a hypodermic needle though a septum, and the ballast bag would inflate. After that, Cell 1 was submerged in $\mathrm{LN}_{2}$, and valve $\mathrm{V} 2$ was opened to draw the gas into the cell. We then close V2 and removed Cell 1 from the plumbing system.

After allowing a few hours for the impurities in the gas mixture to react with $\mathrm{Rb}$ in Cell 1, the radioactive gas was transferred to Cell 2 (1.25" I.D.). Cell 2 was also pre-filled with $\mathrm{Rb}$, and it was the phantom that would be used for imaging studies. As 


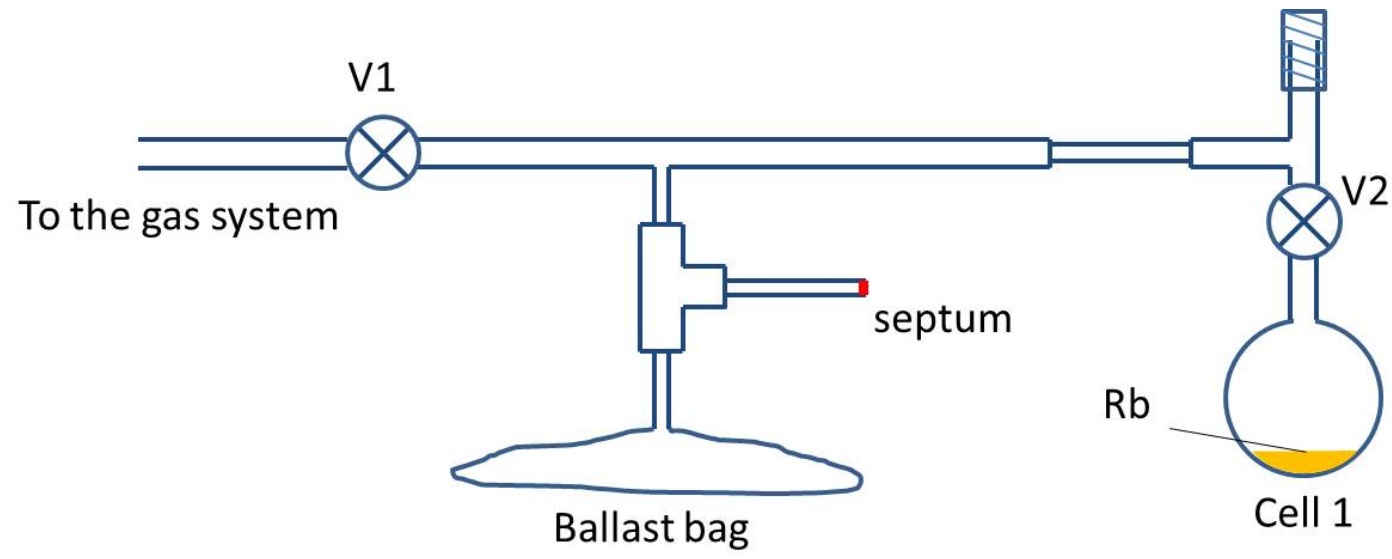

Figure 5.11: Removing the "dirty" gases of the ${ }^{131 \mathrm{~m}}$ Xe gas mixture by sending it into a temporary cell with Rb.

shown in Fig. 5.12, both Cell 1 and Cell 2 were connected to the plumbing system. The plumbing tubes and Cell 2 were roughed out by sequentially opening valve V1 and V3 while actively pumping on the system. After roughing, we typically added about 0.5 Torr of non-radioactive Xe to Cell 2 from the gas system since the amount of radioactive Xe was too small to condense even at LN2 temperature. Cell 2 was then closed and the plumbing tubes were roughed out again. After that, Cell 2 was submerged in $\mathrm{LN}_{2}$ and V2 and V3 was opened to let Xe condense in Cell 2. We noticed that even if we had already added a small amount of non-radioactive Xe, it was still very slow to accumulate ${ }^{131 \mathrm{~m}}$ Xe in Cell 2 just by diffusion. Therefore we developed a "breathing" technique that greatly accelerated the xenon condensation process. Basically, we kept Cell 2 at LN2 temperature while periodically submerging Cell 1 in LN2 and bringing it back to room temperature, which helped the gas to move inside the system. After about 10 "breathing" cycles, over $90 \%$ radioactivity was transferred to Cell 2 . We then closed V2 and added some buffer gas $\left(\mathrm{N}_{2}\right.$ pre-mixed with a few torr of $\left.\mathrm{H}_{2}\right)$ to Cell 2 . Finally, V3 was closed and Cell 2 was removed. The total pressure in Cell 2 at room temperature was about 400 Torr. 


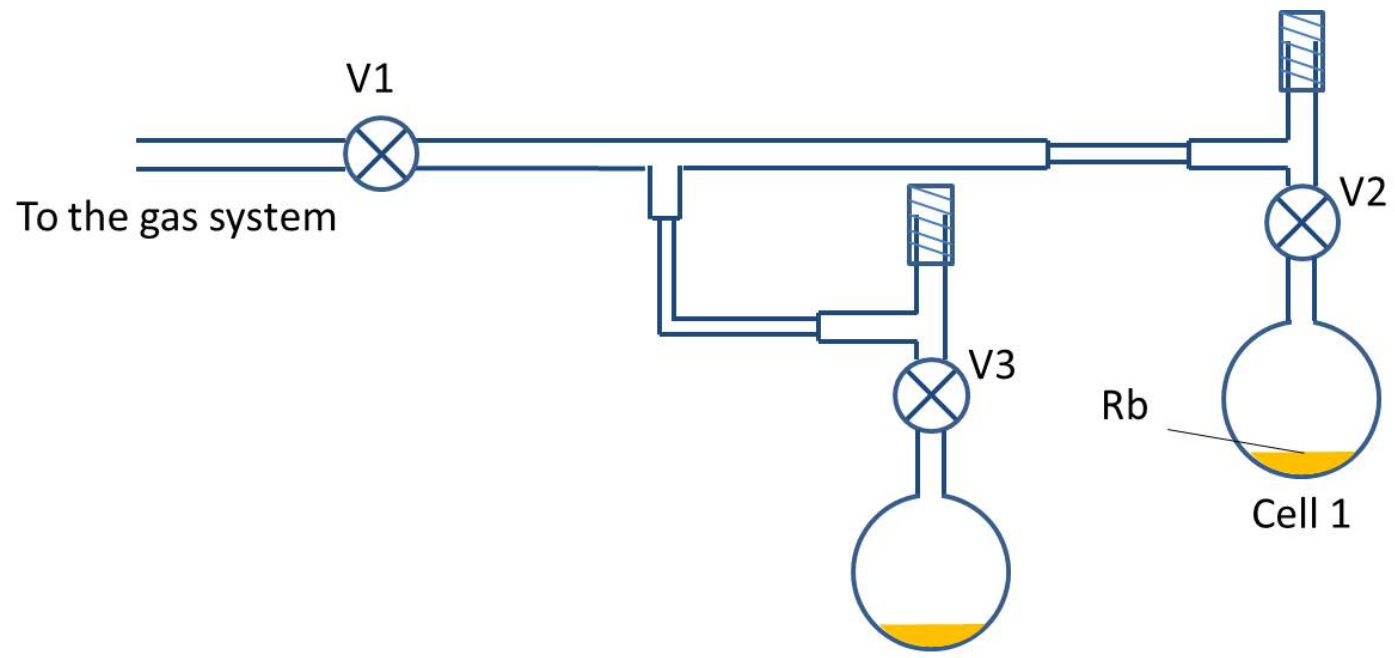

Cell 2

Figure 5.12: Transferring radioactive Xe to a phantom.

\subsection{Imaging Methods}

\subsubsection{Basic PND measurements}

The phantom was polarized using the technique of SEOP. The oven temperature was set at $140^{\circ} \mathrm{C}$ and the phantom was illuminated with a Comet laser (Newport Corporation, Irvine, CA) and a D-Light laser (Raytum Corporation, Manassas, VA). The FWHMs of the two lasers are about $0.2 \mathrm{~nm}$ and $0.3 \mathrm{~nm}$ respectively, and the powers were both measured to be about $20 \mathrm{~W}$.

The $164 \mathrm{keV}$ photon count rate was measured by three detectors. One was placed at the back of the heating oven along the longitudinal direction, and the other two lay in the transverse plane (Fig. 5.7). As shown in Fig. 5.2. $164 \mathrm{keV}$ gammas are emitted preferably in the transverse plane with polarized ${ }^{131 \mathrm{~m}}$ Xe. Therefore the count rate drops in Detector $\# 1$ and increases in Detector $\# 2$ and $\# 3$ as ${ }^{131 \mathrm{~m}}$ Xe becomes polarized.

\subsubsection{NMR Spectroscopy}

The first step in our procedure was to locate the NMR transition frequency of ${ }^{131 \mathrm{~m} X e . ~ A ~}$ weak RF field was applied transverse to the holding field $\mathrm{B}_{0}$ during continuous SEOP. The RF frequency was scanned while the gamma count rate was measured. When 


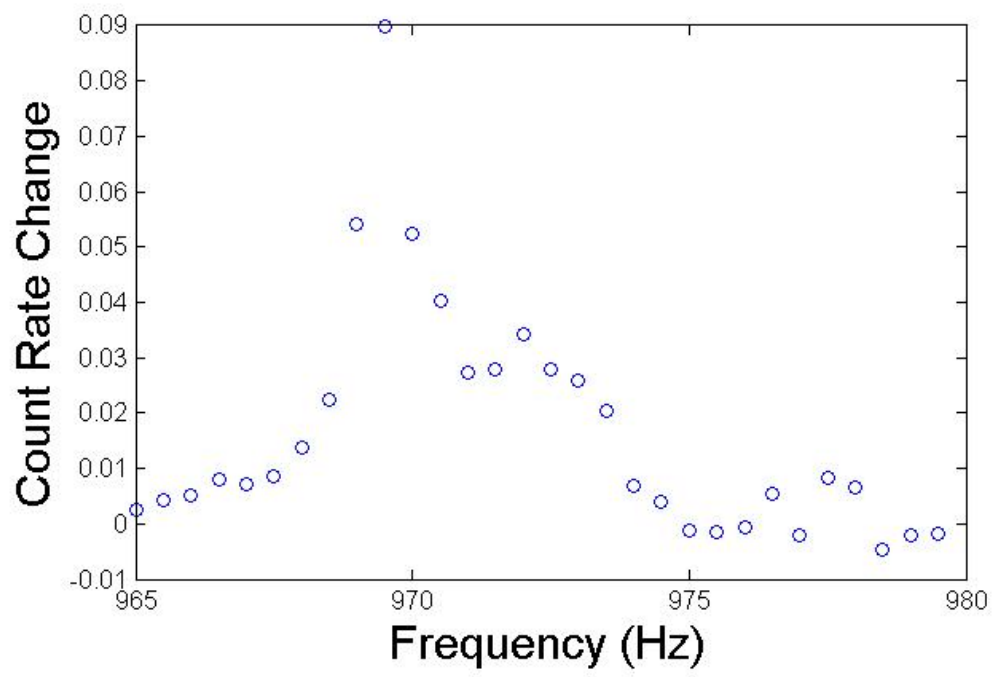

Figure 5.13: Longitudinal gamma ray count rate as a function of the transverse $\mathbf{R F}$ frequency. The count rate change was calculated from the count rates of the longitudinal detector by $\frac{R(f)-R_{0}}{R_{0}}$.

the RF field matched the resonant frequency, the ${ }^{131 \mathrm{~m}}$ Xe polarization was disturbed, resulting in an increase in the longitudinal count rate and a decrease in the transverse count rate. We describe the change of the the gamma count rate by a normalized quantity $\frac{R(f)-R_{0}}{R_{0}}$, where $R(f)$ is the count rate with RF at frequency $\mathrm{f}$, and $R_{0}$ is the count rate with no depolarizing $\mathrm{RF}$. The count rate change measured by the longitudinal detector is plotted in Fig. 5.13. The resonance occurred around $970 \mathrm{~Hz}$ with a holding field of 7 Gauss. The NMR line shown here has a complicated shape, which we speculate to be caused by quadrupole and higher order interactions of the spin- $11 / 2{ }^{131 \mathrm{~m}} \mathrm{Xe}$ nucleus. However, the extraordinary line shape was not observed in Calaprice's early work [12].

\subsubsection{Spin Evolution During SEOP}

The evolution of ${ }^{131 \mathrm{~m}}$ Xe nuclear spin polarization during SEOP was measured as follows: first a resonant RF was broadcast to the phantom to completely depolarize the spins. Then we stopped the RF and immediately started recording the gamma counts as a function of time while the polarization built up. This cycle was repeated many 
times to acquire enough counts to suppress statistical noise. Data were combined by simply adding up the \# of photons that arrived in each time bin. The change of gamma count rate in the longitudinal detector during SEOP is plotted in Fig. 5.14. Similar to before, the change is defined as $\frac{R_{u n}-R(p)}{R_{u n}}$, where $R_{u n}$ is the count rate from unpolarized ${ }^{131 \mathrm{~m}}$ Xe before an SEOP cycle starts, and $\mathrm{R}(\mathrm{p})$ is the count rate from polarized ${ }^{131 \mathrm{~m} X e}$ during SEOP. The count rate reached equilibrium in about 2 mins. The "spin up" time constant $\tau$ was found to be about $33 \mathrm{~s}$ by fitting the data to a model $a\left(1-e^{-t / \tau}\right)+b$. According to the count rate change, the maximum ${ }^{131 \mathrm{~m}}$ Xe polarization was about $57 \%$.

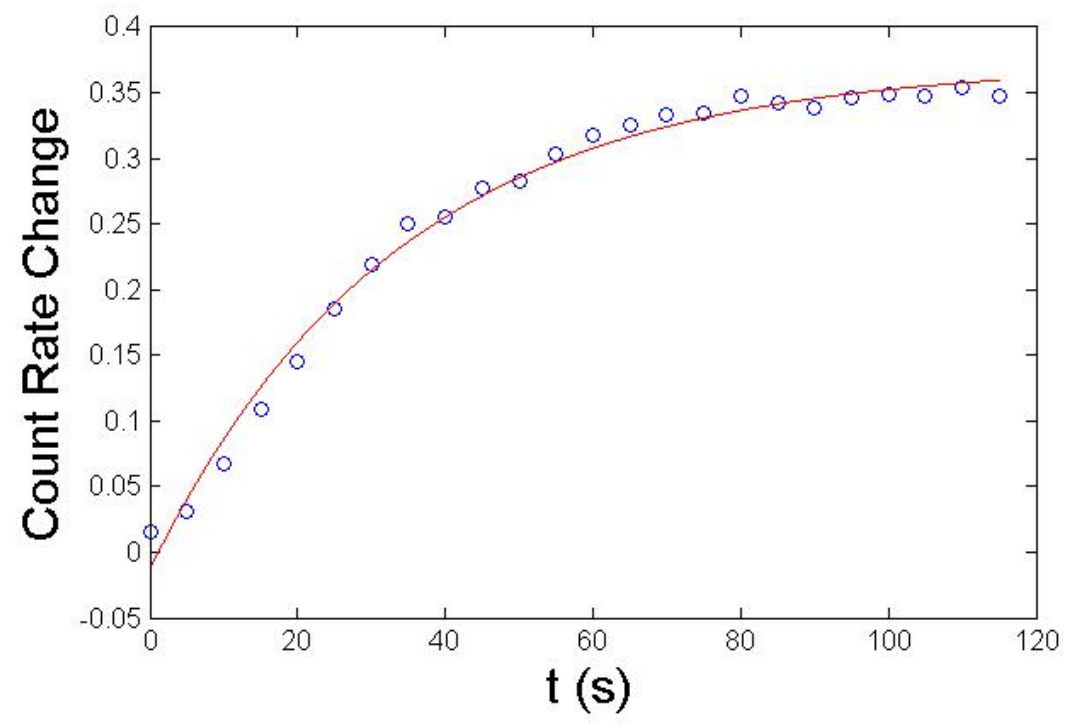

Figure 5.14: Change of the longitudinal gamma ray count rate during SEOP. The count rate change was calculated by $\frac{R_{u n}-R(p)}{R_{u n}}$.

\subsubsection{RF Calibration}

The RF pulses were calibrated to provide specific ${ }^{131 \mathrm{~m}}$ Xe flip angles. One flip angle of special interest to us is 90 degrees, which was used in the CMZ pulse sequence. Once the polarization of the ${ }^{131 \mathrm{~m}} \mathrm{Xe}$ saturated, we sent an RF pulse at the Larmor frequency and measured the $164 \mathrm{keV}$ gamma count rate after excitation. The count rate immediately after the $\mathrm{RF}$ pulse depends on the flip angle. A $90^{\circ}$ flip would result in the maximum count rate in the longitudinal detector. We swept the RF amplitude 
while fixing its duration to $\sim 5 \mathrm{~ms}$. The ratio of the count rate after and before the $\mathrm{RF}$ pulse was fitted to a sinusoid as shown in Fig. 5.15 . The peak of this curve corresponds to a $90^{\circ}$ flip.



Figure 5.15: Ratio of the count rate immediately after and before the RF pulse as a function of $\mathbf{R F}$ amplitude. Data were fit to $a+b \cos (c x+d)$. The $90^{\circ}$ flip angle corresponds to the peak of the curve.

\subsubsection{Free Induction Decay}

The holding field was shimmed as described in section 4.3.5 with a ${ }^{129} \mathrm{Xe}$ cell. Free Induction Decay of ${ }^{131 \mathrm{~m}}$ Xe was measured by sending $90^{\circ} \mathrm{RF}$ pulses to flip polarized ${ }^{131 \mathrm{~m}}$ Xe nuclei to the transverse plane, and immediately taking data using the transverse detectors. We then waited $\sim 2$ mins for the polarization to rebuild, and repeated the measurement. Multiple measurements were taken to improve the SNR. Data were analyzed by first combining them all together (from both detectors and multiple measurements) and then performing a Fourier transform to obtain the frequency spectrum. There was an obvious peak at the expected frequency. Alternatively, we sometimes mixed the combined data to a low frequency and display the time domain signal, as shown in Fig. 5.16. The FID was fit to a damped oscillation model, and the decay time constant was found to be $\sim 150 \mathrm{~ms}$. 




Figure 5.16: ${ }^{131 \mathrm{~m}}$ Xe Free Induction Decay.

We initially expected the $\mathrm{T}_{2}^{*}$ of ${ }^{131 \mathrm{~m}} \mathrm{Xe}$ to be much longer than what we achieved with ${ }^{3} \mathrm{He}$ and ${ }^{129} \mathrm{Xe}$ at a similar holding field since it had a much smaller gyromagnetic ratio. However, the decay time constant of ${ }^{131 \mathrm{~m}}$ Xe was measured to be only about 150 $\mathrm{ms}$, and it seemed to be insensitive to field shimming. This extraordinary behavior led us to reconsider the precession of ${ }^{131 \mathrm{~m}}$ Xe nucleus. As discussed in section 3.1.1. the nuclear precession frequency is determined by the splitting of nuclear energy levels. For a spin-1/2 nucleus, there are only two energy levels and one energy gap, thus there is a single precession frequency. However, for a nucleus with a higher spin $\left({ }^{131 \mathrm{~m} X e}\right.$ is spin 11/2), there are multiple energy levels and the splittings between adjacent levels can be different, which leads to different precession frequencies. Slightly different precession frequencies will cause a dephasing of the spins and therefore a quicker decay of the overall signal. The NMR transition lineshape of ${ }^{131 \mathrm{~m}}$ Xe that we measured also suggested complicated nuclear energy levels (Fig. 5.13).

\subsubsection{CMZ Delay Study}

The key concept of the CMZ pulse sequence is that the spin transverse precession angle can be converted to a polar angle between the spin and $\mathrm{z}$ axis by applying a second 
$90^{\circ} \mathrm{RF}$ pulse, and this polar angle can be controlled by choosing different delays between the first and the second RF (Fig. 5.5). A simple experiment was conducted to demonstrate that this was indeed the case. Two successive $90^{\circ} \mathrm{RF}$ pulses (no gradients applied in between) were applied after ${ }^{131 \mathrm{~m}}$ Xe was polarized to saturation. The count rate of the longitudinal detector was recorded immediately after the second RF. This measurement was then repeated many times with different additional delays $\mathrm{t}_{\text {add }}$. Different $\mathrm{t}_{\text {add }}$ resulted in different polar angles, and the gamma count rate showed a sinusoidal dependence on $t_{a d d}$ as expected. The ratio of the count rate after the second $\mathrm{RF}$ pulse to the count rate before the first $\mathrm{RF}$ is plotted as a function of the extra delay in Fig. 5.17. The period of the oscillation is half of the Larmor period.

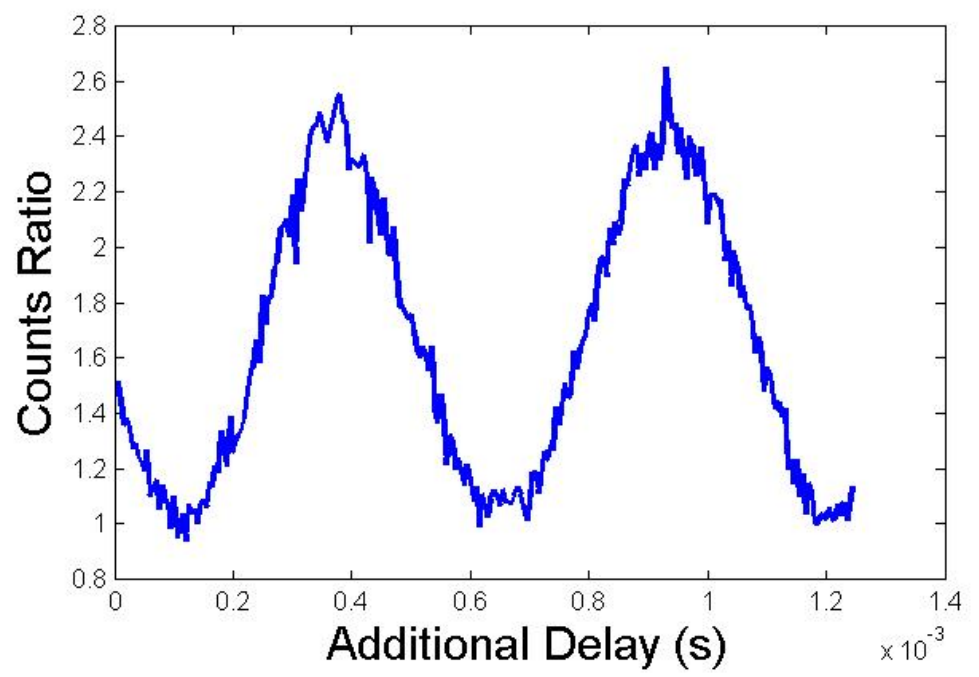

Figure 5.17: Ratio of the gamma count rates as a function of additional delay. The period of the count rate ratio was about $0.52 \mathrm{~ms}$, which was half of the Larmor period $\left(\frac{1}{970 \mathrm{~Hz}}\right)$ of ${ }^{131 \mathrm{~m}} \mathrm{Xe}$.

\subsubsection{Pulse Sequence}

The ${ }^{131 \mathrm{~m}}$ Xe phantom was imaged by a CMZ pulse sequence (point-by-point in $\mathrm{k}$ space) during continuous SEOP. 2D images were acquired without slice selection. A symmetric 32 by $32 \mathrm{k}$-space matrix was covered corresponding to $96 \mathrm{~mm}$ field of view and $3 \mathrm{~mm}$ inplane resolution. The two $90^{\circ} \mathrm{RF}$ pulses (Fig. 5.4) were identical with $\sim 5$ ms duration. 
Long RF pulses were necessary to achieve 90 degree flip angles because of the small gyromagnetic ratio of ${ }^{131 \mathrm{~m}}$ Xe. As shown in Eq. 5.10, count rates need to be acquired with two different delays to construct $\mathrm{k}$ space data. We used $35 \mathrm{~ms}$ and $(35+0.13) \mathrm{ms}$ delays respectively for $\mathrm{R}$ and $\mathrm{R}$ '. Imaging gradients were applied immediately after the first $\mathrm{RF}$ and finished within the $\sim 35 \mathrm{~ms}$ delay time. All three detectors took data for $2 \mathrm{~s}$ after the second RF. We then waited $\sim 100 \mathrm{~s}$ for the ${ }^{131 \mathrm{~m}}$ Xe polarization to build up again before taking the next measurement. Each $\mathrm{k}$ space point was measured with both delays before we proceeded to the next point in $\mathrm{k}$ space. 2048 excitations were needed for such an imaging procedure, which took $\sim 57 \mathrm{hrs}$. We note here that most of the time needed was just for polarizing ${ }^{131 \mathrm{~m}} \mathrm{Xe}$, and was not required by the imaging technique itself. The total data taking time was $\sim 1.1 \mathrm{hrs}$ for each detector. The 164 $\mathrm{keV}$ gamma count rate during the procedure was about $1.1 \mathrm{kHz}$.

\subsubsection{Auxiliary Measurements}

Since the aforementioned imaging procedure takes a long time, the holding field needs to be monitored so that corrections can be applied for the field drift. However, unlike the case of ${ }^{129} \mathrm{Xe}$ imaging described in section 4.4 .2 , the transverse relaxation of ${ }^{131 \mathrm{~m}} \mathrm{Xe}$ was very fast and the holding field cannot be measured accurately with FIDs. Thus we measured the holding field using a completely different approach. A small SEOP oven was placed $20 \mathrm{~cm}$ above the ${ }^{131 \mathrm{~m}}$ Xe phantom, in which a $1.5 "$ diameter cell (which we called the field monitoring cell) with about $2.5 \mathrm{~atm}$ of $\mathrm{N}_{2}$ and some Rb was installed. The oven temperature was set at $150^{\circ} \mathrm{C}$, and the cell was illuminated by a spectrally broadband laser (FWHM $\sim 2 \mathrm{~nm}, 25 \mathrm{~W}$ ) known as the "FAP" (Coherent Laser Inc, Santa Clara, CA). A pair of small transverse coils and a photodiode were set up for EPR measurements (section 3.2 and 3.3.3.1). EPR measurements were performed with the field monitoring cell after data acquisition finished with the ${ }^{131 \mathrm{~m}}$ Xe phantom in each polarization cycle. The Rb Zeeman transition frequency in the field monitoring cell was located, which could be used to determine the holding field strength. We assumed that the field disturbance varied slowly in space so that the field drift at the ${ }^{131 \mathrm{~m}}$ Xe phantom was identical to that at the field monitoring cell. The holding field drift during the whole CMZ imaging procedure was shown in Fig. 5.18 . 


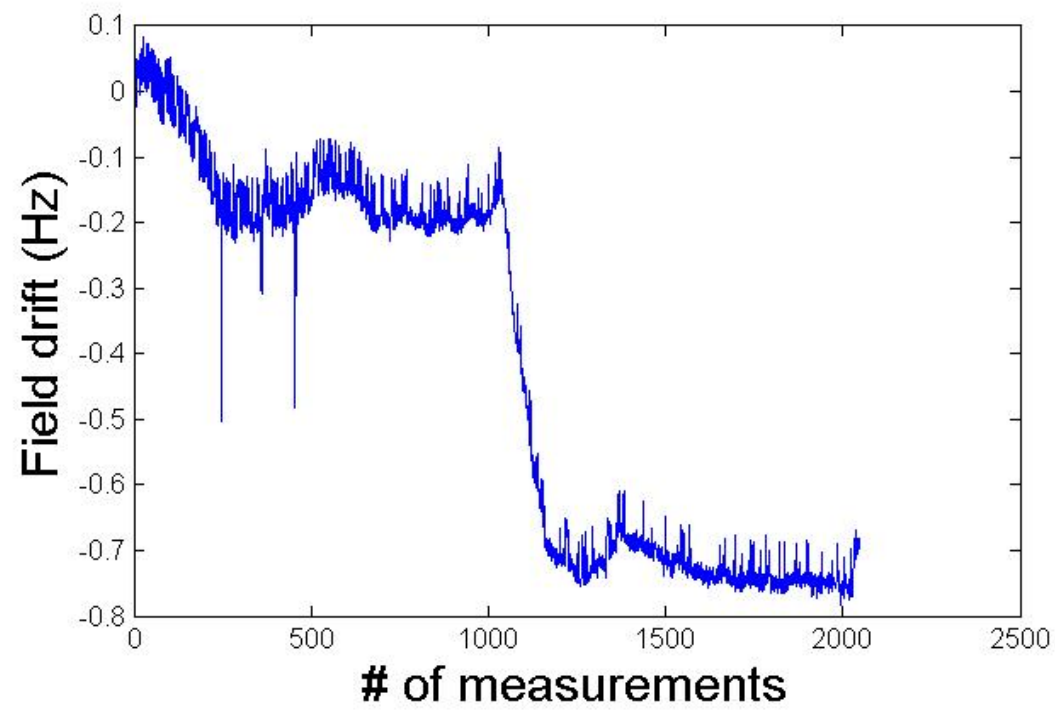

Figure 5.18: Holding field drift measured by EPR during the whole CMZ procedure. The unit of the field drift is in ${ }^{131 \mathrm{~m}}$ Xe precession frequency.

\subsubsection{Image Reconstruction}

Data from the three detectors were analyzed separately. For each set of data, we combined the count rates measured with two different delays and constructed a 32 by $32 \mathrm{k}$ space matrix. Since the total imaging time was not negligible compared with the ${ }^{131 \mathrm{~m}} \mathrm{Xe}$ half life (12 days), adjustment was made to account for the decay of ${ }^{131 \mathrm{~m} X \mathrm{X}}$ nuclei. The DC offset due to the $a_{0}$ term was removed. However, there was also a slight slope in the $\mathrm{k}$ space matrix, which we believe was caused by the drop of ${ }^{131 \mathrm{~m}} \mathrm{Xe}$ polarization during the long imaging procedure due to the deterioration of the phantom. This slope was taken off empirically. The field drift effect was corrected by multiplying each $\mathrm{k}$ space point by a factor $e^{i 2 \pi\left(2 \Delta f t_{\text {delay }}\right)}$, where $\Delta f$ is the holding drift in the unit of ${ }^{131 \mathrm{~m}}$ Xe precession frequency. The $\mathrm{k}$ space matrix of the longitudinal detector is shown in Fig. 5.19, and the noise of which is mainly due to the uncertainty in count rates. 2D Fourier transform was then performed to generate the image.

A total of three images were generated. Image1 (Fig. 5.20(a) from the longitudinal detector had a better SNR than image2 and image3 from the transverse ones. These 

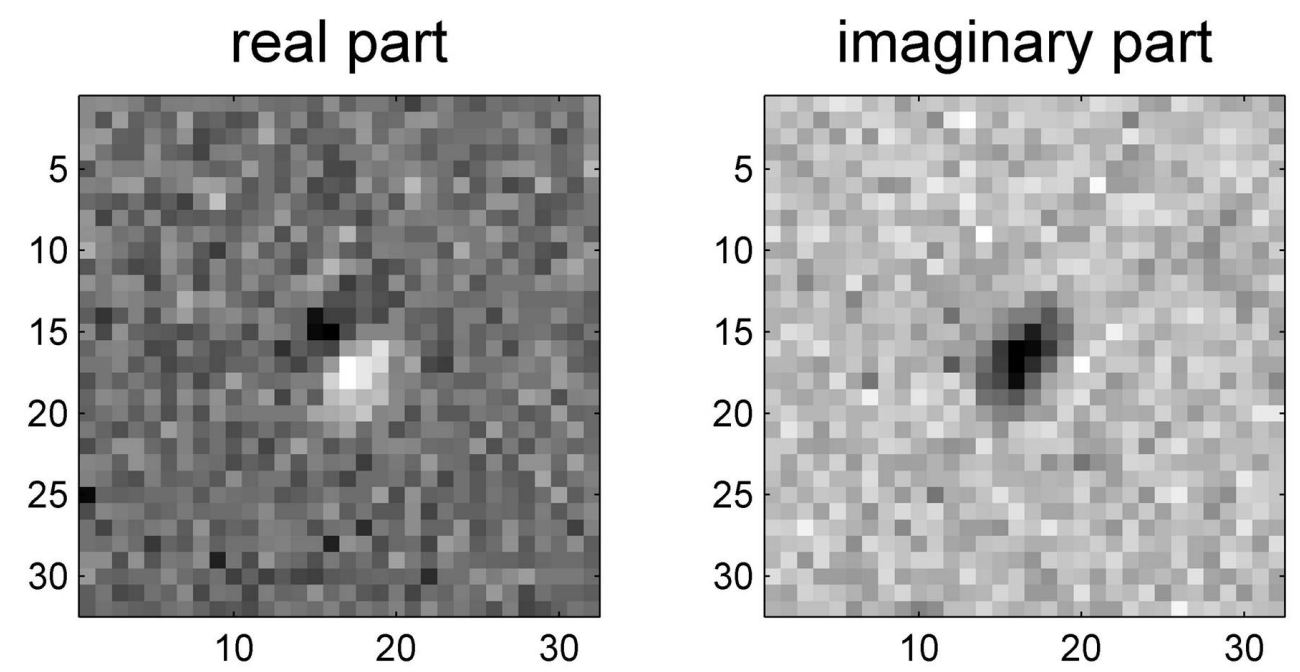

Figure 5.19: Corrected k space data of the longitudinal detector.

images were combined together to further increase the SNR. First the images from the two transverse detectors were added up coherently to generate image23 (Fig. 5.20(b)). We then combined image 1 and image 23 by weighting them according to the square of the corresponding SNRs and adding them up incoherently. The final image (image123) is shown in Fig. 5.21 .

\subsection{Results and Discussions}

Although Fig. 5.21 is only a crude image and took a long time to collect, it is the first image ever acquired with a combination of MR spatial encoding and gamma detection. Moreover, non-oscillating data were collected by the novel CMZ sequence in both the longitudinal direction and the transverse plane.

Both higher resolution and better SNR are needed to improve the image quality. The gyromagnetic ratio of ${ }^{131 \mathrm{~m}} \mathrm{Xe}$ is very low, and it limited the resolution of Fig. 5.21 to $3 \mathrm{~mm}$ with the gradients available in our system. However, with stronger imaging gradients, finer resolution can be achieved. To obtain a better SNR, more counts need to be collected. One obvious approach is to increase the radioactive dose. The activity of our phantom at the time of imaging was only about $0.27 \mathrm{mCi}$. Even if we increase the dose by a factor of ten, it is still a small amount in the context of nuclear imaging. We also mentioned in previous discussions that useful counts can be collected in almost any 


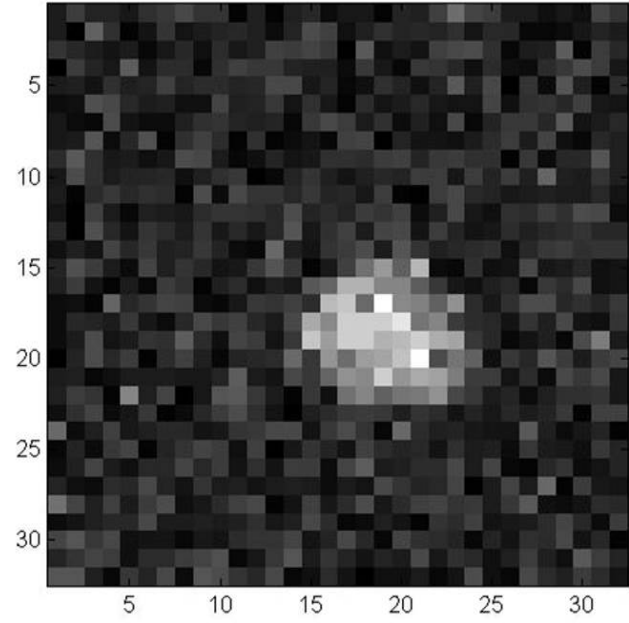

(a) Image from the longitudinal detector.

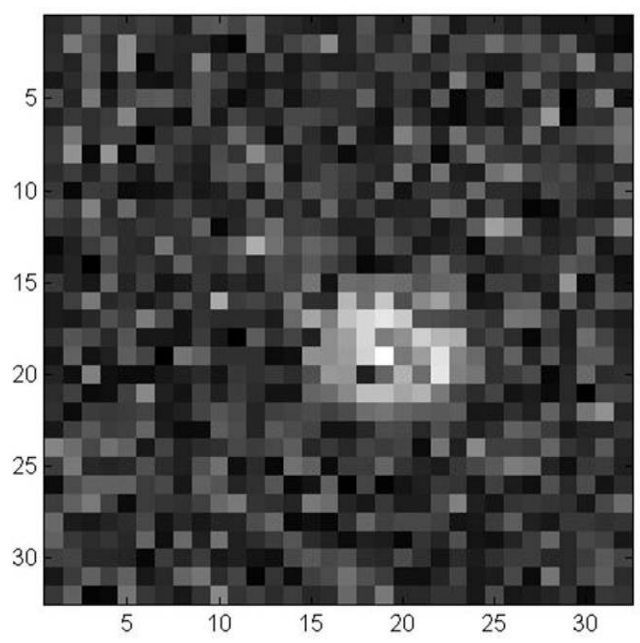

(b) Combined image from the transverse detectors.

Figure 5.20: Images acquired by the longitudinal and transverse detectors. The spatial resolution is $3 \mathrm{~mm}$.



Figure 5.21: PNI image of a $\sim 3 \mathbf{c m}$ diameter sphere. The image shown here is generated by combining one image collected in the longitudinal direction and two images acquired in the transverse plane. The pixel size is $3 \mathrm{~mm}$ by $3 \mathrm{~mm}$. The SNR is about 6 .

direction in PNI. Therefore simply adding more detectors to cover a bigger solid angle would also help. Of course the images need to be weighted properly when combined. 
Yet another possibility of acquiring more counts is to increase the data taking time in each SEOP cycle. In our experiment we only collected counts for $2 \mathrm{~s}$ after the second $\mathrm{RF}$. The reason is that spins with different polar angles evolve very differently in the presence of SEOP, thus the data taking time must be short compared with the SEOP rate. However, if we temporarily block the pumping laser after the second RF pulse, spins will evolve towards the unpolarized state in a more uniform fashion, which might allow a longer period of time for productive data taking.

Improving SEOP of the radioactive nuclei would also help. It is likely that with more laser power, a higher saturation polarization can be achieved with a shorter "spin up" time constant. On the one hand, the fraction of "useful" counts (the $a_{2}$ term) increases. On the other hand, ${ }^{131 \mathrm{~m}}$ Xe can be polarized to saturation faster thus the whole imaging procedure will take less time.

We discussed in section 4.5.3 that for in-vivo rodent lung ${ }^{129} \mathrm{Xe} \mathrm{MRI}$, a reservoir of hyperpolarized ${ }^{129} \mathrm{Xe}$ and a rodent ventilation machine need to be involved. It is a

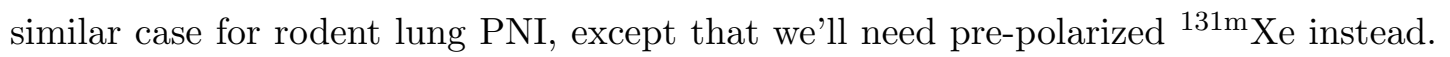
A typical ventilatory cycle is $600 \mathrm{~ms}$ with a $300 \mathrm{~ms}$ breath hold [103. If we use 2.7 $\mathrm{mCi}$ activity for each breath hold, which is ten times larger than what we had in the phantom, $300 \mathrm{~ms}$ will be long enough for data taking. Since it takes two breaths to measure each $\mathrm{k}$ space point, the time needed to cover a 32 by 32 matrix is about 20 mins. The exhaled radioactive xenon can be recycled for future use. Alternatively, it might be possible to send the recycled ${ }^{131 \mathrm{~m}}$ Xe back to the Xe polarizer continuously during PNI, where it can be repolarized and used again within the same imaging procedure.

\subsection{Other Applications of PND}

PND is a platform technique that leads to a series of novel experiment approaches that greatly exceed the sensitivity of current methods.

One example is Rabi precession spectroscopy. If a continuous resonant RF field is applied to polarized nucleus, the spins will rotate around the transverse $\mathrm{B}_{1}$ field while precessing at the Larmor frequency around the holding field. The rotation around the transverse $B_{1}$ field is called Rabi precession. Since the $B_{1}$ field is usually much smaller than $\mathrm{B}_{0}$ field, Rabi precession is much slower than Larmor Precession. As a result, it is difficult and even impossible to detect Rabi precession using a coil by electromagnetic 
induction. However, Rabi precession can be easily observed by radiation detection. Suppose we have a detector installed in the longitudinal direction, the angle between the spins and the detector (polar angle) changes due to rabi procession, and the radiation count rate changes accordingly. The spectrum of ${ }^{131 \mathrm{~m}}$ Xe Rabi precession generated from the longitudinal detector count rate is shown in Fig. 5.22.

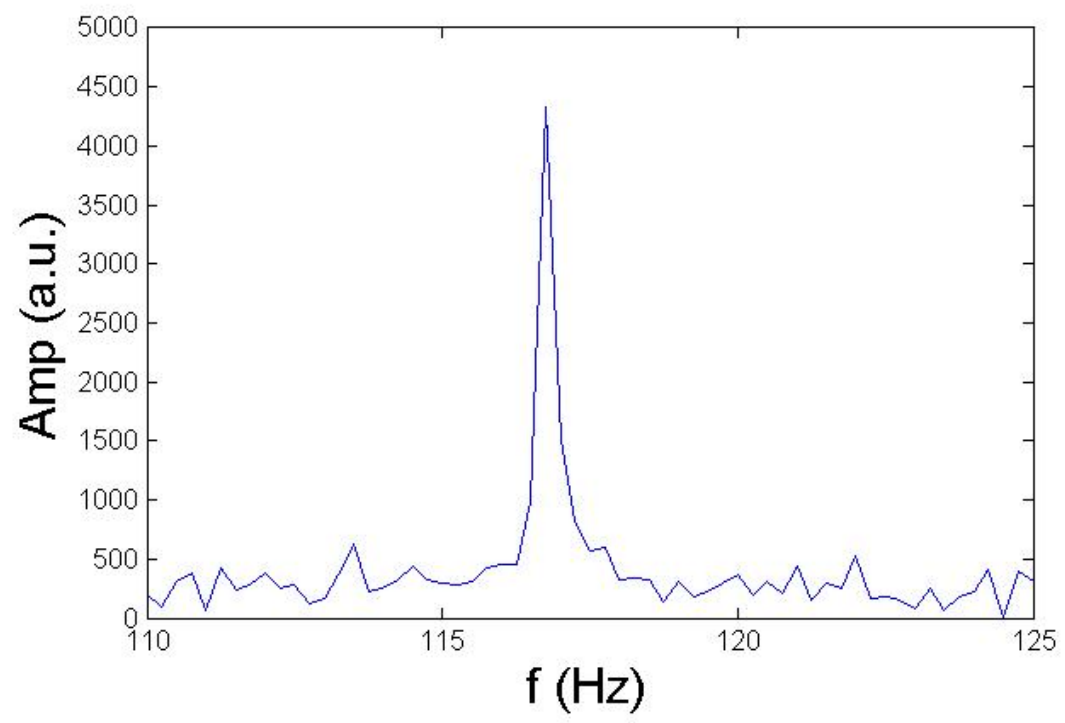

Figure 5.22: Rabi precession spectrum of ${ }^{131 \mathrm{~m}} \mathrm{Xe}$ measured by a longitudinal detector. The $\mathrm{B}_{0}$ field is about 7 Gauss and the $\mathrm{B}_{1}$ field is about 0.5 Gauss.

Another example is $\mathrm{T}_{1 \rho}$ measurement, which is also called a spin-locking measurement. In a $T_{1 \rho}$ experiment, the spins are first flipped to the transverse plane by a $90^{\circ} \mathrm{RF}$ pulse. Then another continuous $\mathrm{RF}$, which is referred to as the spin-locking field, is applied with $\mathrm{B}_{1}$ field in parallel with the spins in the rotating frame. The spin polarization undergoes relaxation in the presence of the spin-locking field with a decay time constant $\mathrm{T}_{1 \rho}$. The spin-locking field $\mathrm{B}_{1}$ plays a similar role as $\mathrm{B}_{0}$ for the relaxation of unflipped spins in the lab frame. $\mathrm{T}_{1 \rho}$ relaxation depends on the strength of the $\mathrm{B}_{1}$ field, which is usually called $\mathrm{T}_{1 \rho}$ dispersion. The conventional way to measure $\mathrm{T}_{1 \rho}$ is to stop the spin-locking field after a time $\mathrm{t}$ and measure the signal size from a transverse detection coil, and then fit it to a model $M(t)=M_{0} e^{t / T_{1 \rho}}$, where $\mathrm{M}_{0}$ is the signal induced by the initial transverse magnetization without any spin-locking. The evolution 
of magnetization cannot be measured directly during spin-locking since an oscillating $\mathrm{B}_{1}$ field is applied. However, if we measure the $\mathrm{T}_{1 \rho}$ of an appropriate nucleus using the technique of PND, not only will the sensitivity be greatly increased, but the damped spin precession can also be measured directly during spin-locking. A single PND $\mathrm{T}_{1 \rho}$ measurement is shown in Fig. 5.23, and a dispersion curve is shown in Fig. 5.24.

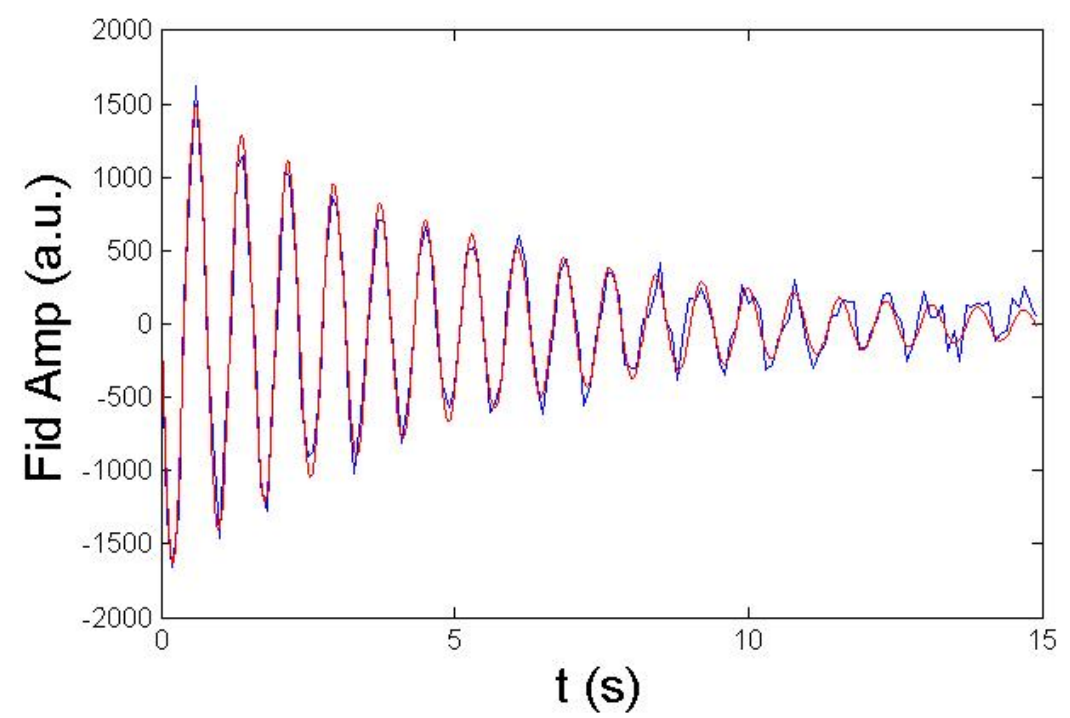

Figure 5.23: Spin precession and decay during a ${ }^{131 \mathrm{~m}} \mathbf{X e} \mathbf{T}_{1 \rho}$ measurement. The oscillation frequency is mixed down for display here. The locking field $\mathrm{B}_{1}=180$ mGauss, and $\mathrm{T}_{1 \rho}$ is found to be $5.2 \mathrm{~s}$ by a damped oscillation fit.

The technique of PND can also be used as a non-imaging diagnostic technique. Hyperpolarized ${ }^{129} \mathrm{Xe}$ has been recognized as a biochemical sensor, since its MR properties, such as the gyromagnetic ratio, $T_{1}, T_{2}$ and $T_{1 \rho}$ are sensitive to the biochemical environment. However, in some circumstances it is very difficult to introduce enough hyperpolarized Xe to the organs of interest, and the signals are quite weak. On the other hand, if we use hyperpolarized ${ }^{131 \mathrm{~m}} \mathrm{Xe}$ and PND, only tiny quantities of the radioactive nucleus are needed to generate a decent signal. Moreover, some experiment methods uniquely related to radiation detection can be applied, from as simple as a direct $\mathrm{T}_{1}$ measurement to the Rabi precession and $\mathrm{T}_{1 \rho}$ measurements that we mentioned above. 


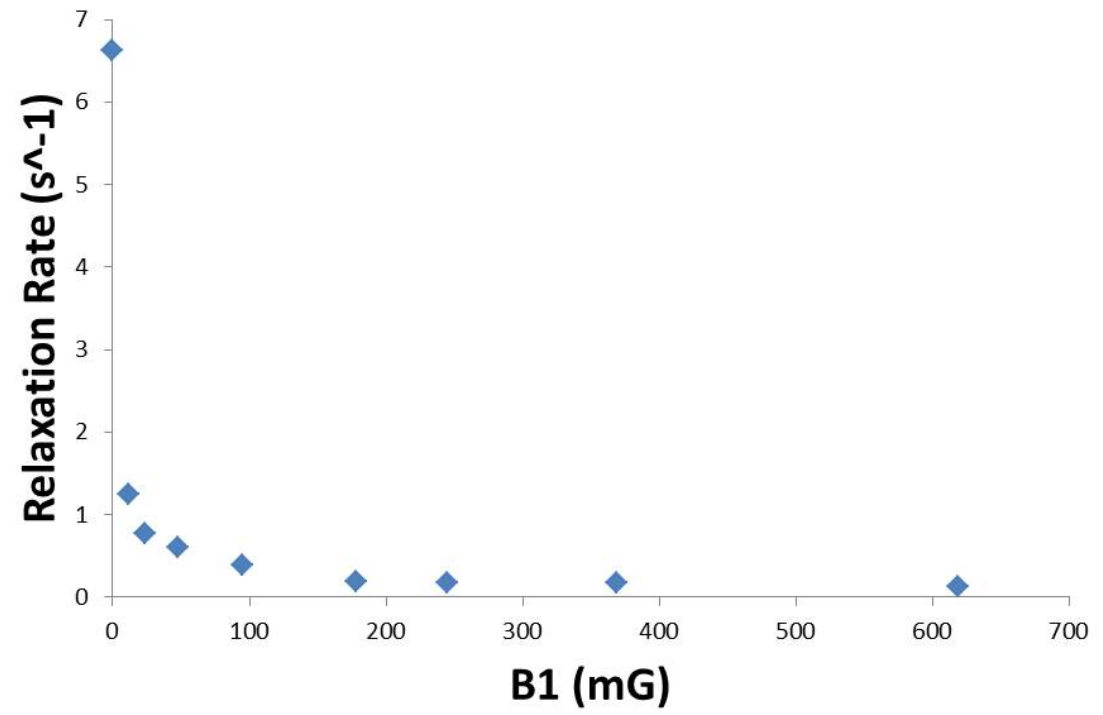

Figure 5.24: $\mathbf{T}_{1 \rho}$ dispersion curve of ${ }^{131 \mathrm{~m}} \mathbf{X e}$. The relaxation rate $\frac{1}{T_{1 \rho}}$ is plotted as a function of the locking field strength. 


\section{6 \\ Conclusion}

\subsection{Summary}

The technique of Spin-Exchange Optical Pumping (SEOP) is widely used nowadays to produce large volumes of hyperpolarized noble gases. We discussed SEOP on a introductory level and presented our study of ${ }^{129} \mathrm{Xe}$ polarization with different gas compo-

sitions. ${ }^{129}$ Xe polarization was calibrated directly or indirectly using the technique of Electron Paramagnetic Resonance (EPR). We then described our home-built low-field MRI system in great detail, which was built with off-the-shelf electronics and circular coils. Using a fully phase encoded pulse sequence and with some auxiliary measurements, we acquired a high quality image with ${ }^{129} \mathrm{Xe}$ at low field. We then introduced the novel techniques of Polarized Nuclear Imaging (PNI) and Polarized Nuclear Detection (PND), which make use of the asymmetry of radioactive emissions of certain polarized radioactive nuclei. We studied the MR properties of ${ }^{131 \mathrm{~m}}$ Xe by detecting the anisotropic gamma emissions. We also incorporated the gamma detection system into our low field MRI scanner and collected an image with the CMZ pulse sequence that we had specially developed for PNI.

\subsection{Future Directions}

The novel techniques of PNI and PND combine the great flexibility of MR and the high sensitivity of radiation detection, and have great potential for a lot of applications. We've studied PNI and PND in-vitro, the next step would be to carry out in-vivo studies on small animals. 
The first step would be to study the Xenon distribution after injection into a subject by either intravenous injection or inhalation. Radioactive xenon doesn't need to be polarized, and its distribution can be observed using a gamma camera.

Second, a ${ }^{131 \mathrm{~m}}$ Xe polarizer, which is able to polarize and dispense the radioactive isotope, needs to be built. Since the amount of Xe atoms involved is so small, the design of the polarizer can be relatively simple, using either SEOP or dynamic nuclear polarization techniques.

Third, the polarized ${ }^{131 \mathrm{~m}} \mathrm{Xe}$ can be introduced into the subject, and PNI and PND studies can be carried out. PND focuses on measuring the change of MR properties $\left(\mathrm{T}_{1}\right.$, $\mathrm{T}_{2}$, Larmor frequency, ...) of ${ }^{131 \mathrm{~m}} \mathrm{Xe}$ in normal and abnormal tissues using radiation detection. PNI focuses on collecting an image of the tissue of interest. A ventilation machine or continuous intravenous injection system may need to be involved to keep the amount of polarized ${ }^{131 \mathrm{~m}}$ Xe above a certain level during a long imaging procedure. Multiple detectors should be set up to cover a bigger solid angle for more effective data collection. Also with a higher activity, it might be possible to collect an image using the Fast Low Angle Shot (FLASH) pulse sequence.

Finally, although we used ${ }^{131 \mathrm{~m}}$ Xe for our preliminary studies, other radioactive isotopes should also be considered, one such example is ${ }^{79 m} \mathrm{Kr}$. Different atoms have different physical and chemical properties, and might be suited for certain niche applications. 


\section{References}

[1] P. C. Lauterbur. Image Formation by Induced Local Interactions: Examples Employing Nuclear Magnetic Resonance. Nature, 242, 1973. 1

[2] P. Mansfield, A. A. Maudsley. Medical Imaging by NMR. British Journal of Radiology, 50, 1977. 1]

[3] R. Damadian, M. Goldsmith, L.Minkoff. NMR in cancer: XVI: FONAR image of the live human body. Physiol Chem PHys, 9, 1977. 1

[4] M.S. Albert, G.D. Cates, B. Driehuys, W. Happer, B. Saam, C. S. Springer Jr AND A. WishniA. Biological magnetic resonance imaging using laser-polarized ${ }^{129}$ Xe. Nature, 370, 1994. 2, 59

[5] S.Appelt, A.Ben-Amar Baranga, C.J. Erickson, M.V. Romalis, A. R. Young, W. Happer. Theory of spin-exchange optical pumping of ${ }^{3} \mathbf{H e}$ and ${ }^{129}$ Xe. Phys. Rev. A, 58(2), 1998. 2, 7, 18

[6] A. Ben-Amar Baranga, S. Appelt, M. V. Romalis, C. J. Erickson, A. R. Young, G. D. Cates, W. Happer. Polarization of ${ }^{3}$ He by Spin Exchange with Optically Pumped Rb and K Vapors. Physical Review Letters, 80(13), 1998. 2, 19

[7] Thad G. Walker, William Happer. Spin-exchange optical pumping of noble-gas nuclei. Rev. Mod. Phys., 69:629-642, 1997. 2, 13, 17, 19

[8] Gordon D. Cates Jr., Bastiaan Driehuys, William Happer, Hunter Middleton, Eli Miron, Brian SaAm, Daniel Walter. High Volume 
hyperpolarizer for spin-polarized noble gas. U.S. Patent No. 5,642,625, 1996. 3

[9] Gordon D. Cates Jr., Bastiaan Driehuys, William Happer, Eli Miron, Brian SaAm, DANiel WAlter. Cryogenic accumulator for spin-polarized xenon-129. U.S. Patent Nos. 5,809,801, 5,860,295, 1997. 3

[10] I. C. Ruset, S. Ketel, F.W. Hersman. Optical Pumping System Design for Large Production of Hyperpolarized 129Xe. PHYSICAL REVIEW LETTERS, 96, 2006. 3

[11] Schrank G, Ma Z, Schoeck A, SaAm B. Characterization of a lowpressure high-capacity ${ }^{129} \mathrm{Xe}$ flow-through polarizer. Phys. Rev. A, 80:063424, 2009. 3, 70

[12] F. P. Calaprice, W. Happer, D.F. Schreiber, M. M. Lowry, E. Miron, X. ZEnG. Nuclear Alignment and Magnetic Moments of ${ }^{133} \mathrm{Xe},{ }^{133} \mathbf{X e}^{m}$ and ${ }^{131} \mathbf{X e}^{m}$ by Spin Exchange with Optically Pumped ${ }^{87} R b$. Phys. Rev. Lett., 54:174-177, 1985. 3, 24, 86, 87, 103

[13] Anthony PL, Et.AL. Determination of the neutron spin structure function. Physical Review Letters, 71(7), 1993. 5

[14] Riordan S, ET.AL. Measurements of the electric form factor of the neutron up to $\mathrm{Q} 2=3.4 \mathrm{GeV} 2 \mathrm{using}$ the reaction $3 \mathrm{He}\left(\mathrm{e}, \mathrm{e}^{\prime} \mathrm{n}\right) \mathrm{pp}$. Physical Review Letters, 105(26), 2010. 5

[15] F. D. Colegrove, L. D. Schearer, G. K. Walters. Polarization of ${ }^{3} \mathbf{H e}$ Gas by Optical Pumping. Physical Review, 132(6), 1963. 5

[16] Jaideep Singh, P. A. M. Dolph, W. A. Tobias, T. D. Averett, A. Kelleher, K. E. Mooney, V. V. Nelyubin, Yunxiao Wang, Yuan Zheng, G. D. Cates. The Development of High-Performance Alkali-Hybrid Polarized He-3 Targets for Electron Scattering. submitted to Phys. Rev. C, 2014. 5, 7, 22

[17] William Happer. Optical Pumping. Phys. Mod. Phys, 44(2), 1972. 7 
[18] Kilian TJ. Thermionic Phenomena Caused by Vapors of Rubidium and Potassium. Phys. Rev., 27:578-587, 1926. 7

[19] Peter Dolph. High-Performance Nuclear-Polarized ${ }^{3}$ He Targets for Electron Scattering Based on Spin-Exchange Optical Pumping. PhD thesis, University of Virginia, 2010. 9, 13, 14, 23, 37, 45

[20] M. E. Wagshul, T. E. Chupp. Optical Pumping of high-density Rb with a broadband dye laser and GaAlAs diode laser arrays: Application to ${ }^{3}$ He polarization. Phys. Rev. A, 40(8), 1989. 10, 12

[21] M. V. Romalis, E. Miron, G. D. Cates. Pressure broadening of $\mathbf{R b} \mathbf{D}_{1}$ and $\mathrm{D}_{2}$ lines by ${ }^{3} \mathrm{He},{ }^{4} \mathrm{He}, \mathrm{N}_{2}$, and $\mathrm{Xe}$ : Line cores and near wings. Phys. Rev. A, 56(6), 1997. 13,52

[22] B. Larson, O. Hausser. Optical pumping of $\mathbf{R b}$ in the presence of high-pressure ${ }^{3}$ He buffer gas. Phys. Rev. A, 44(5), 1991. 12

[23] I. A. Nelson, T. G. Walker. Rb-Xe spin relaxation in dilute Xe mixtures. Phys. Rev. A, 65(012172), 2001. 14

[24] Stephen John KadleceK. Spin Relaxation in Alkali Vapors. PhD thesis, University of Wisconsin, 1999. 14

[25] X. Zeng, Z. Wu, T. Call, E. Miron, D. Schreiber, W. Happer. Experimental determination of the rate constants for spin exchange between optically pumped $\mathrm{K}, \mathrm{Rb}$, and $\mathrm{Cs}$ atoms and ${ }^{129} \mathrm{Xe}$ nuclei in alkalimetal-noble-gas van der Waals molecules. Phys. Rev. A, 31(1), 1985. 15. 21

[26] G. D. Cates, R. J. Fitzgerald, A. S. Barton, P. Bogorad, M. Gatzke, N. R. Newbury, B. SaAm. Rb- ${ }^{129}$ Xe spin-exchange rates due to binary and three-body collisions at high Xe pressures. Phys. Rev. A, 45(7), 1992. 15, 21

[27] B. Driehuys, G. D. Cates, E. Miron, K. Sauer, D. K. Walter, W. HAPPER. High-volume production of laser-polarized ${ }^{129}$ Xe. Appl. Phys. Lett., 69(1668), 1996. 15, 21 
[28] Charles V. Ricea, Daniel Raftery. Rubidium-xenon spin exchange and relaxation rates measured at high pressure and high magnetic field. J. Chem. Phys., 117(12), 2002. 15, 21

[29] Iulian C. Ruset. Hyperpolarized ${ }^{129}$ Xe Production and Applications. PhD thesis, University of New Hampshire, 2005. 15

[30] L. Wilmer Anderson, Francis M. Pipkin, James C. Baird. Hyperfine Structure of Hydrogen, Deuterium, and Tritium. Physicsl Review, 120(4), 1960. 16

[31] M. E. Wagshul, T. E. Chupp. Laser optical pumping of high-density Rb in polarized ${ }^{3}$ He targets. Phys. Rev. A, 49(5), 1994. 17

[32] B. Chann, E. Babcock, L. W. Anderson, T. G. Walker. Skew light propagation in optically thick optical pumping cells. Physicsl Review A, 66(033406), 2002. 18

[33] J. Singh. Some notes on Alkali- ${ }^{3}$ He SEOP. Technical Report 1.10, 2006. 18

[34] D. K. Walter, W. Happer, T. G. Walker. Estimates of the relative magnitudes of the isotropic and anisotropic magnetic-dipole hyperfine interactions in alkali-metal-noble-gas systems. Phys. Rev. A, 58(5), 1998. 19

[35] W. Happer, E. Miron, S. Schaefer, D. Schreiber, W. A. Van WijngaArden, X. Zeng. Polarization of the nuclear spins of noble-gas atoms by spin exchange with optically pumped alkali-metal atoms. Phys. Rev. A, 29(6), 1984. 19, 20

[36] Yuan-Yu Jau, Nicholas N. Kuzma, William Happer. High-field measurement of the ${ }^{129} \mathrm{Xe}-\mathrm{Rb}$ spin-exchange rate due to binary collisions. Phys. Rev. A, 66(052710), 2002. 19

[37] Yuan-Yu Jau, Nicholas N. Kuzma, William Happer. Magnetic decoupling of ${ }^{129} \mathrm{Xe}-\mathrm{Rb}$ and ${ }^{129} \mathrm{Xe}-\mathrm{Cs}$ binary spin exchange. Phys. Rev. A, 67(002720), 2003. 19 
[38] N. R. Newbury, A. S. Barton, G. D. Cates, W. Happer, H. Middleton. Gaseous ${ }^{3} \mathrm{He}^{3} \mathrm{He}$ magnetic dipolar spin relaxation. Phys. Rev. A, 48(6), 1993. 22

[39] R. L. Gamblin, T. R. Carver. Polarization and Relaxation Processes in ${ }^{3}$ He Gas. Physical Review, 138(4A), 1965. 22

[40] L. D. Schearer, G. K. Walters. Nuclear Spin-Lattice Relaxation in the Presence of Magnetic-Field Gradients. Physical Review, 139(5A), 1965. 22

[41] G.D.Cates, D.J.White, Ting-Ray Chien, S.R.Schaefer, W.Happer. Spin relaxation in gases due to inhomogeneous static and oscillating magnetic fields. Phys. Rev. A, 38(10), 1988. 22, 64, 65

[42] Barbe R., Leduc M., Laloe F. J. Physique, 35:935-951, 1974. 22

[43] G. D. Cates. SLAC-Report 444. Technical report, 1993. 22

[44] Ming F. Hsu, G. D. Cates, I. Kominis, I. A. Aksay, and D. M. Dabbs. Sol-gel coated glass cells for spin-exchange polarized ${ }^{3}$ He. Appl. Phys. Lett., 77, 2000. 22

[45] B. Chann, I. A. Nelson, L.W. Anderson, B. Driehuys, T. G. Walker. ${ }^{129}$ Xe-Xe Molecular Spin Relaxation. Phys. Rev. Lett, 88(11), 2002. 23

[46] H. Watts. Diffusion in Multicomponent Gaseous Mixtures. Can. J. Chem, 43, 1965. 23

[47] Scott Rohrbaugh. Evidence for Relaxation of ${ }^{129}$ Xe By Paramagnetic Impurities on RbH Surfaces. PhD thesis, University of Virginia, 2008. 23, 47

[48] Scott Rohrbaugh, H. T. J. Wang, J. Singh, W. A. Tobias, G. D. Cates. Magnetic decoupling of ${ }^{129} \mathrm{Xe}$ nuclear spin relaxation due to wall collisions with RbH and RbD coatings. Phys. Rev. A, 86(043413), 2012. 23

[49] M. Kitano, M. Bourzutschky, F. P. Calaprice, J. Clayhold, W. HapPer, M.Musolf. Measurement of magnetic dipole moments of ${ }^{129} \mathbf{X e}^{m}$ and ${ }^{131} \mathbf{X e}^{m}$ by spin exchange with optically pumped Rb. Phys. Rev. C, 34:1974-1979, 1986. 24, 87 
[50] Z. Wu,W. Happer, M. Kitano, J. Daniels. Experimental studies of wall interactions of adsorbed spin-polarized ${ }^{131} \mathrm{Xe}$ nuclei. Phys. Rev. A, 42, 1990. 24

[51] Thad G. Walker. Estimates of spin-exchange parameters for alkalimetalCnoble-gas pairs. Phys. Rev. A, 40, 1989. 25

[52] I. I. Rabi, N. F. Ramsey, J. Schwinger. Use of Rotating Coordinates in Magnetic Resonance Problems. Rev. Mod. Phys., 26:167-171, 1954. 29

[53] Abragam, A. Principles of Nuclear Magnetism. Oxford University Press, 1961. 30

[54] F. BLOCH. Nuclear Induction. Physical Review, 70(7), 1946. 33

[55] M. V. Romalis, G. D. Cates. Accurate ${ }^{3}$ He polarimetry using the Rb Zeeman frequency shift due to the Rb-3He spin-exchange collisions. Phys. Rev. A, 58(4), 1998. 34, 36, 41, 54

[56] G. Breit, I. I. Rabi. Measurement of Nuclear Spin. Phys. Rev., 38:20822083, 1931. 35

[57] J. D. JACKson. Classical Electrodynamics, 3rd edition. Wiley, 1998. 36, 51,67

[58] Earl Babcock, Ian A. Nelson, Steve Kadlecek, Thad G. Walker. ${ }^{3}$ He polarization-dependent EPR frequency shifts of alkali-metal- ${ }^{3} \mathrm{He}$ pairs. Phys. Rev. A, 71, 2005. 36, 54

[59] Z. L. MA, E. G. Sorte, B. SAam. Collisional ${ }^{3}$ He and ${ }^{129}$ Xe Frequency Shifts in Rb-Noble-Gas Mixtures. Phys. Rev. Lett., 106(193005), 2011. 37 , 41,54

[60] Mikhail V. Romalis. Laser Polarized ${ }^{3}$ He Target Used for a Precision Measurement of the Neutron Spin Structure. PhD thesis, Princeton University, 1997. 45

[61] Fain S, Schiebler ML, McCormack DG, Parraga G. Imaging of lung function using hyperpolarized helium-3 magnetic resonance imaging: 
Review of current and emerging translational methods and applications. Journal of Magnetic Resonance Imaging, 32(6), 2010. 59

[62] Mugler JP 3RD, Altes TA. Hyperpolarized ${ }^{129}$ Xe MRI of the human lung. J Magn Reson Imaging, 37:313-331, 2013. 59

[63] Walker TG, Happer W. Spin-exchange optical pumping of noble-gas nuclei. Rev Mod Phys, 69:629-642, 1997. 59

[64] SaAm BT, Yablonskiy DA, Kodibagkar VD, Leawoods JC, Gierada DS, Cooper JD, Lefrak SS, Conradi MS. MR Imaging of Diffusion of ${ }^{3} \mathbf{H e}$ Gas in Healthy and Diseased Lungs. Magn Reson Med, 44:174-179, 2000. 59

[65] Yablonskiy DA, Sukstanskit AL, Leawoods JC, Gierada DS, BretThorst GL, Lefrak SS, CoOper JD, ConRAdi MS. Quantitative in vivo assessment of lung microstructure at the alveolar level with hyperpolarized ${ }^{3}$ He diffusion MRI. Proc Natl Acad Sci USA, 99:3111-3116, 2002. 59

[66] Mugler JP 3rd, Wang C, Miller GW, Cates GD Jr, Mata JF, Brookeman JR, De Lange EE, Altes TA. Helium-3 Diffusion MR Imaging of the Human Lung Over Multiple Time Scales. Acad Radiol, 15:693-701, 2008. 59

[67] Mair RW, Wong GP, Hoffmann D, Hurlimann MD, Patz S, Schwartz LM, Walsworth RL. Probing Porous Media with Gas Diffusion NMR. Phys Rev Letters, 83:3324-3327, 1999. 59

[68] Kuder TA, Bachert P, Windschuh J, Laun FB. Diffusion pore imaging by hyperpolarized xenon-129 nuclear magnetic resonance. Phys Rev Letters, 111:028101, 2013. 59

[69] Wong GP, Tseng CH, Pomeroy VR, Mair RW, Hinton DP, Hoffmann D, Stoner RE, Hersman FW, Cory DG, Walsworth RL. A System for Low Field Imaging of Laser-Polarized Noble Gas. J Magn Reson, 141:217-227, 1999. 59, 60, 64, 75, 82 
[70] Cross AR, McDonald M, Parra Robles J, Santyr GE. Laser-polarized ${ }^{129} \mathrm{Xe}$ NMR at $1.88 \mathrm{~T}$ and $8.5 \mathrm{mT}$ : a signal-to-noise ratio comparison. J Magn Reson, 162:241-249, 2003. 59

[71] Parra-Robles J, Cross AR, Santyr GE. Theoretical signal-to-noise ratio and spatial resolution dependence on the magnetic field strength for hyperpolarized noble gas magnetic resonance imaging of human lungs. Med Phys, 32:221-229, 2005. 59

[72] Parra-Robles J, Cross AR, Santyr GE. Erratum: "Theoretical signalto-noise ratio and spatial resolution dependence on the magnetic field strength for hyperpolarized noble gas magnetic resonance imaging of human lungs". Med Phys, 33:1525-1526, 2006. 59

[73] Venkatesh AK, Zhang AX, Mansour J, Kubatina L, Oh CH, Blasche G, Selim Unl M, Balamore D, Jolesz FA, Goldberg BB, Albert MS. MRI of the lung gas-space at very low-field using hyperpolarized noble gases. Magn Reson Imaging, 21:773-776, 2003. 60

[74] Durand E, Guillot G, Darrasse L, Tastevin G, Nacher PJ, Vignaud A, Vattolo D, Bittoun J. CPMG measurements and ultrafast imaging in human lungs with hyperpolarized helium-3 at low field (0.1 T). Magn Reson Med, 47:75-81, 2002. 60, 80

[75] Patz S, Muradian I, Hrovat Mi, Ruset IC, Topulos G, Covrig SD, Frederick E, Hatabu H, Hersman FW, Butler JP. Human Pulmonary Imaging and Spectroscopy with Hyperpolarized ${ }^{129} \mathrm{Xe}$ at $\mathbf{0 . 2 T}$. Acad Radiol, 15:713-727, 2008. 60

[76] Owers-Bradley JR, Fichele S, Bennattayalah A, McGloin CJ, Bowtell RW, Morgan PS, Moody AR . MR tagging of human lungs using hyperpolarized ${ }^{3}$ He gas. J Magn Reson Imaging, 17:142-146, 2003. 60 . 80

[77] Tseng CH, Wong GP, Pomeroy VR, Mair RW, Hinton DP, Hoffmann D, Stoner RE, Hersman FW, Cory DG, Walsworth RL. Low-Field MRI of Laser Polarized Noble Gas. Phys Rev Lett, 81:3785-3788, 1998. 60 
[78] Bidinosti CP, Choukeife J, Tastevin G, Vignaud A, Nacher PJ. MRI of the lung using hyperpolarized ${ }^{3} \mathrm{He}$ at very low magnetic field $(3 \mathbf{m T})$. MAGMA, 16:255-258, 2004. 60, 80

[79] Mair RW, Hrovat Mi, Patz S, Rosen MS, Ruset IC, Topulos GP, Tsai LL, Butler JP, Hersman FW, Walsworth RL. ${ }^{3}$ He Lung Imaging in an Open Access, Very-Low-Field Human MRI System. Magn Reson Med, 53:745-749, 2005. 60, 82

[80] Ruset iC, Tsai Ll, Mair RW, Patz S, Hrovat Mi, Rosen MS, Muradian I, NG J, Topulos GP, Butler JP, Walsworth RL, Hersman FW. A system for open-access ${ }^{3} \mathrm{He}$ human lung imaging at very low field. Concepts Magn Reson Part B Magn Reson Eng, 29:210-221, 2006. 60, 82

[81] Tsai Ll, Mair RW, Rosen MS, Patz S, Walsworth RL. An openaccess, very-low-field MRI system for posture-dependent ${ }^{3} \mathrm{He}$ human lung imaging. J Magn Reson, 193(2):274 - 285, 2008. 60, 64

[82] Shao W, Wang G, Fuzesy R, Hughes EW, Chronik BA, Scott GC, Conolly SM, Macovski A. Low readout field magnetic resonance imaging of hyperpolarized xenon and water in a single system. Appl Phys Lett, 80:2032-2034, 2002. 60

[83] Dana A. Shea, Daniel Morgan. The Helium-3 Shortage: Supply, Demand, and Options for Congress. CRS Report for Congress, 2010. 60

[84] Swanson SD, Rosen MS, Agranoff BW, Coulter KP, Welsh RC, Chupp TE. Brain MRI with laser-polarized ${ }^{129}$ Xe. Magn Reson Med, 38:695-698, 1997. 60

[85] Swanson SD, Rosen MS, Coulter KP, Welsh RC, Chupp TE. Distribution and Dynamics of Laser-Polarized ${ }^{129}$ Xe Magnetization In Vivo. Magn Reson Med, 42:1137-1145, 1999. 60

[86] Kilian W, Seifert F, Rinneberg H. Dynamic NMR spectroscopy of hyperpolarized ${ }^{129} \mathrm{Xe}$ in human brain analyzed by an uptake model. Magn Reson Med, 51:843-847, 2004. 60 
[87] Driehuys B, M?ller He, Cleveland ZI, Pollaro J, Hedlund LW. Pulmonary Perfusion and Xenon Gas Exchange in Rats: MR Imaging with Intravenous Injection of Hyperpolarized ${ }^{129}$ Xe. Radiology, 252:386-393, 2009. 60

[88] Mugler JP 3Rd, Altes TA, Ruset IC, Dregely IM, Mata JF, Miller GW, Ketel S, Ketel J, Hersman FW, Ruppert K. Simultaneous magnetic resonance imaging of ventilation distribution and gas uptake in the human lung using hyperpolarized xenon-129. Proc Natl Acad Sci USA, 107:21707-21712, 2010. 60

[89] Dregely I, Mugler JP 3rd, Ruset IC, Altes TA, Mata JF, Miller GW, Ketel J, Ketel S, Distelbrink J, Hersman FW, Ruppert K. Hyperpolarized Xenon-129 Gas-Exchange Imaging of Lung Microstructure: First Case Studies in Subjects With Obstructive Lung Disease. J Magn Reson Imaging, 33:1052-1062, 2011. 60

[90] P. A. M. Dolph, J. Singh, T. Averett, A. Kelleher, K. E. Mooney, V. Nelyubin, W. A. Tobias, B. Wojtsekhowski, G. D. Cates. Gas dynamics in high-luminosity polarized He-3 targets using diffusion and convection. PHYSICAL REVIEW C, 84(6), 2011. 61

[91] E. Mark Haacke, Robert W. Brown, Michael R. Thompson, Ramesh Venkatesan. Magnetic Resonance Imaging Physical Principles and Sequence Design. Wiley-Liss, 1999. 64

[92] Driehuys B, Cates GD, Miron E, Sauer K, Walter DK, Happer W. High volume production of laser polarized ${ }^{129}$ Xe. Appl Phys Lett, 69:16681670, 1996. 70

[93] Ruset IC, Ketel S, Hersman FW. Optical Pumping System Design for Large Production of Hyperpolarized ${ }^{129}$ Xe. Phys Rev Lett, 96:053002, 2006. 70

[94] CARL M. Characterizing Microstructure of Porous Media Using Noble-GasDiffusion MRI at Short Time Scales. PhD thesis, University of Virginia, 2008. 71 
[95] Jackson Ji, Meyer CH, Nishimura DG, Macovski A. Selection of a Convolution Function for Fourier Inversion Using Gridding. IEEE Trans Med Imag, 10:473-478, 1991. 78

[96] Gravina S, Cory DG. Sensitivity and Resolution of Constant-Time Imaging. J Magn Reson B, 104:53-61, 1994. 81

[97] Brown TR, Kincaid BM, Ugurbil K. NMR chemical shift imaging in three dimensions. Proc Natl Acad Sci USA, 79, 1982. 81

[98] Miller KW,REO NV, Uiterkamp AJMS, Stengle DP, Stengle TR, Williamson KL. Xenon NMR:Chemical shifts of a general anesthetic in common solvents, proteins, and membranes. Proc Natl Acad Sci USA, 78:4946-4949, 1981. 82

[99] Mugler JP 3Rd, Altes TA, Ruset IC, Miller GW, Mata JF, Qing K, Tsentalovich I, Hersman FW, Ruppert K. Image-based Measurement of $\mathbf{T}_{2}^{*}$ for Dissolved-phase Xe-129 in the Human Lung . Proc Intl Soc Mag Reson Med, 20:1347, 2012. 82

[100] Tsai LL, Mair RW, Li CH, Rosen MS, Patz S, Walsworth RL. Posturedependent human He-3 lung imaging in an open-access MRI system: Initial results. Acad Radiol., 15(6):728-739, 2008. 82

[101] Chen XJ, Hedlund LW, Moller HE, Chawla MS, Maronpot RR, JohnSON GA. Detection of emphysema in rat lungs by using magnetic resonance measurements of ${ }^{3}$ He diffusion. Proc Natl Acad Sci USA, 97:1147811481, 2000. 82

[102] Fox MS, Ouriadov A, SAntyr GE. Comparison of hyperpolarized ${ }^{3}$ He and ${ }^{129} \mathrm{Xe}$ MRI for the measurement of absolute ventilated lung volume in rats. Magn Reson Med, 71:1130-1136, 2014. 82

[103] Nouls J1, Fanarjian M, Hedlund L, Driehuys B. A Constant-Volume Ventilator and Gas Recapture System for Hyperpolarized Gas MRI of Mouse and Rat Lungs. Concepts Magn Reson Part B Magn Reson Eng, 39B:78-88, 2011. 83,112 
[104] N. R. Steenberg. The Angular Distribution of $\gamma$-Radiation from Aligned Nuclei. Proc. Phys. Soc. A, 65:791-800, 1952. 86

[105] T. Yamazaki. . Nuclear Data Tables, A3(1), 1967. 86

[106] M. Kitano, F. P. Calaprice, M. L. Pitt, J. Clayhold, W. Happer, M. Kadar-Kallen, M. Musolf, G. Ulm, K. Wendt, T. Chupp, J. Bonn, R. Neugart, E. Otten, H. T. Duong. Nuclear Orientation of Radon Isotopes by Spin-Exchange Optical Pumping. Phys. Rev. Lett., 60:21332136, 1988. 87 